\title{
Gait Features and Their Relationships to Physiological Attributes of Multiple Sclerosis
}

\author{
A Dissertation \\ Presented to \\ the faculty of the School of Engineering and Applied Science \\ University of Virginia
}

\author{
in partial fulfillment \\ of the requirements for the degree \\ Doctor of Philosophy \\ by
}

Asma Qureshi

May 2017 


\section{APPROVAL SHEET}

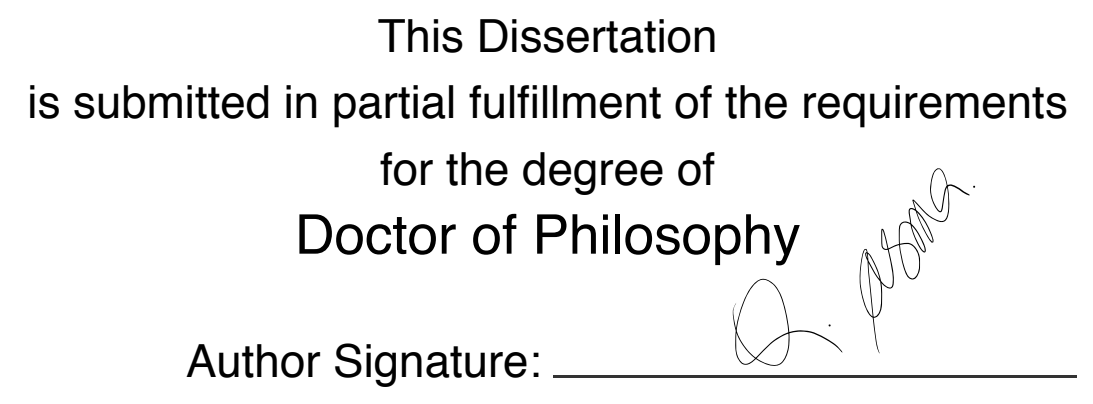

This Dissertation has been read and approved by the examining committee:

Advisor: Maite Brandt-Pearce

Committee Member: Stephen G. Wilson

Committee Member: John C. Lach

Committee Member: Daniel S. Weller

Committee Member: Myla D. Goldman

Committee Member: Stephen D. Patek

Accepted for the School of Engineering and Applied Science:

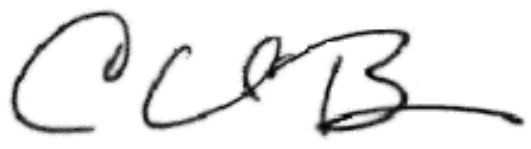

Craig H. Benson, School of Engineering and Applied Science

May 2017 
(C) 2017 Asma Qureshi 
To Riaz and Saira 


\section{Acknowledgements}

I would like to sincerely thank my advisor, Dr. Maite Brandt-Pearce, for her relentless support, understanding, and patience. It was her persistent encouragement which pushed me to be my best and guided me to the right direction whenever I was directionless. She inspired me not only as a role model but also as a friend. Her camaraderie, exemplary work ethic, determination, and emphasis on participating in group meetings, seminars, and social activities fostered my multi-dimensional technical skills and shaped me as an independent researcher. At the same time, it instilled team spirit, mutual respect, empathy, flexibility, and numerous other personal and interpersonal skills in me, which I will cherish for the rest of my life.

I am grateful to all the committee members for advising me and being open to discussions and ideas. Each member made vital contributions to this research in some way, and it has been a privilege working with them. Dr. Myla Goldman played a pivotal role in adding the clinical facet to the research and kept me moving forward with her appreciation. Signal-processing discussions with Dr. Daniel Weller were very useful and his mentoring during the supervised teaching class gave me an exceptional, hands-on teaching experience. Dr. Stephen Patek was always available to give me guidance at critical points. Dr. John Lach welcomed me in his group meetings and encouraged research collaborations with his team. I am thankful to my chair, Dr. Stephen Wilson. His role as a member of my advisory committee has been the key.

I would also like thank Drs. Nathan Swami, John Lach, and Stephen Wilson for their roles as administrative faculty over the years, setting up the graduate council, and giving graduate students the opportunity to be a part of building an interactive, social environment in the department. Additionally, I am appreciative of the support

and services of the folks in the School of Engineering and Applied Science and In- 
ternational Studies Office. I also want to thank the National Science Foundation for their financial support, primarily under grant IIS-1231712.

I can not thank enough my parents for their perseverance and love. It was their unprecedented support that allowed me to pursue my dreams. I would also like to express my gratitude to my siblings, brother-in-law, all of my teachers, group-mates, and friends, for their smiles and moral support that gave me motivation to complete the doctoral program. 


\section{Abstract}

The developments in signal processing, data mining, and machine learning tools, supported by the advent of body sensor networks, capable of collecting high-precision and continuous data in a non-invasive fashion, resulted in great interest in manipulating gait data into equivalent objective markers for identification of pathological gait or other functional and cognitive impairments, monitoring ailments, evaluating the efficacies of the treatments, guiding recovery and rehabilitation, sports training, and enabling self-monitoring. Gait analysis has become a crucial assessment tool in medicine. It is used to provide new insights to help understand various human movement patterns and fluctuations in them corresponding to pathologies and neurological conditions affecting motor and/or cognitive functions. Multiple sclerosis (MS) is an example of such disorders. People with MS represent a heterogeneous cohort with a broad spectrum of symptoms. Gait dysfunction is a common finding, but one with varied etiology.

Assessments of MS-associated cognitive and motor disability, the disease course and its progression, and decision-making regarding disease-modifying treatments and symptoms management, are based on clinical observations, comprised of outcomes of physical examinations and medical imaging, and patient-rated questionnaires. Being reliant on physicians' judgment in interpreting imaging and clinical outcomes, affected by the differences among individuals regarding the notion of disability or improvements, time-consuming, imprecise, having limited sensitivity to subtle changes in gait, and low variance in ratings are some of the drawbacks of current subjective evaluations. Moreover, patient-reported outcomes (PROs) are subject to response shifts due to changes within individuals over time regarding health standards, and could lead to confusing findings and discrepancies between expected and observed 
indicators, negatively impacting disease prognosis. We intend to augment existing information, on-going research, and currently-used speed and distance-based clinical assessments, for a neurologic condition, with new, objective, and clinically meaningful anchors. Although our goals are motivated for a target application (finding physiologically meaningful gait features for assessing functional quality in MS using inertial gait data), our test measures could be adopted for gait assessments and monitoring in other neurological disorders, balance, stability, and fall risk prediction, and general health and wellness applications.

We derive inertial features using angular rate gait data collected using a body sensor node for improved gait assessment with three goals - (i) using variations in gait features over time, i.e., gait dynamics, to remove the inter-subject variability and guide personalized assessments in neurology-affected locomotion, aging, or chronic diseases, (ii) finding computationally efficient and robust gait features that neither require identification of exact gait cycles nor need a large dataset to capture gait deterioration and make physical sense, (iii) translating pathology-induced fluctuations into frequency-domain features to identify the impact of MS on important gait phases. We include gait variables for comparable controls in the study to determine the unique and overlapping features.

We use a class of data mining to generate models estimating the relationships between the inertial measures and clinical assessments and identifying the physiological significance of the feature space to make it accessible to the doctors and allow patient's engagement. The relationships between the inertial and clinical measures also help to identify the clusters of gait variables, besides and beyond what are identified using a traditional disability assessment scale, to confirm whether the scale is the best way to categorize persons with MS into different disability levels based on gait impairment. 
To conclude, we use inertial measures to verify three hypotheses - (i) pathologic gait in MS is restricted and, thus, is less variable in comparison to a healthy gait, (ii) distinct types of MS disability introduces disturbance in various gait components, and (iii) changes in gait variables over time carry additional information about the disease status. We believe that knowledge of our test measures will improve our capacity to monitor the disease and its progression, evaluate the effectiveness of the treatments, improve and tailor subjective assessments based on individual needs, and guide self-management of symptoms. 


\section{Contents}

1 INTRODUCTION 1

1.1 Research Motivation . . . . . . . . . . . . . . . . 3

1.2 Societal Motivation . . . . . . . . . . . . . . . . . . . . 6

1.3 Broader Impacts . . . . . . . . . . . . . . . . . . . . . . . . . . . . 7

1.4 Our Contribution . . . . . . . . . . . . . . . . . . . 7

1.5 Literature Review . . . . . . . . . . . . . . . . . . . . . . . . . . . . . . . . . . .

1.6 Dissertation Outline ................... 15

2 HILBERT-HUANG TRANSFORM 16

2.1 Introduction . . . . . . . . . . . . . . . . . . . . . 17

2.2 Background . . . . . . . . . . . . . . . . . . . . . . . . . . . . . . .

2.3 Intrinsic Mode Function . . . . . . . . . . . . . . . . . . . . . . . 19

2.4 Empirical Mode Decomposition . . . . . . . . . . . . . . . 20

2.4.1 Sifting Process and its Instability . . . . . . . . . . . . 21

2.4.2 Selective Extrema Analysis . . . . . . . . . . . . . . . 22

2.4.3 Setting the Threshold . . . . . . . . . . . . . 26

2.4.4 Mirror Extension for Boundary Condition . . . . . . . . . . 26

2.4.5 Stopping Criterion . . . . . . . . . . . . 26

2.4.6 Modified Algorithm . . . . . . . . . . . . . . . . . . 27

2.5 The Hilbert Spectrum . . . . . . . . . . . . . . . . . . . 30

2.6 Previous Applications and Modifications of the HHT . . . . . . . 33

2.7 Summary . . . . . . . . . . . . . . . . . 34

3 CLINICAL MEASURES FOR MS ASSESSMENTS 36

3.1 Introduction . . . . . . . . . . . . . . . . . 36

3.2 Types of Clinical Data . . . . . . . . . . . . . . . . . . . . . . . . . . . . . 37

3.2.1 Physician-rated Scores . . . . . . . . . . . . . . . . 37

3.2.2 Patient-reported Outcomes . . . . . . . . . . . . . . 39

3.2.3 Physiological Significance of Gait Features . . . . . . . . . . . 40

3.2.4 Quantitative Walking Tests ... . . . . . . . . . . . 41

3.3 Previous Work Correlating Gait and Clinical Metrics . . . . . . . . . 41

3.4 Summary . . . . . . . . . . . . . . . . . . . . 42 
4 DATA COLLECTION AND PRE-PROCESSING 43

4.1 Body Sensor Network . . . . . . . . . . . . . . . . . . 43

4.2 Study Subjects and Protocol . . . . . . . . . . . . . . . . 44

4.3 Gait Cycle Components . . . . . . . . . . . . . . . . . 45

4.4 Gait Time Series . . . . . . . . . . . . . . . . . . 46

4.5 Summary . . . . . . . . . . . . . . . . . . . . 49

5 STATISTICAL ANALYSIS $\quad 50$

5.1 Spearman Correlation Coefficient . . . . . . . . . . . . 50

5.2 Linear Regression Model . . . . . . . . . . . . . . . . . . . . . . 52

5.3 Summary . . . . . . . . . . . . . . . . . . . 53

6 GRADIENT OF THE STRIDE TIME $\quad 54$

6.1 Stride Time Standard Deviation . . . . . . . . . . . . . . 54

6.2 Gradient of Stride Time Variability over Six Minutes . . . . . . . . 56

6.3 Clinical Significance of Stride Time Gradient . . . . . . . . . . . 62

6.3 .1 Statistical Analysis . . . . . . . . . . . . . . 62

6.3 .2 Results and Discussions _. . . . . . . . . . . . . . 64

6.4 Summary . . . . . . . . . . . . . . . . . 70

7 KERNEL DENSITY ESTIMATION 72

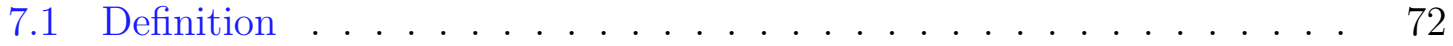

7.1 .1 Bandwidth Selection . . . . . . . . . . . . . . 73

7.2 Kernel Density Estimates of Gait Data . . . . . . . . . . . . 74

7.3 Interpretation of the KDE Peak . . . . . . . . . . . 77

7.4 Clinical Significance of the KDE Peaks . . . . . . . . . . . 80

7.4.1 Statistical Analysis . . . . . . . . . . . . . . . 80

7.4 .2 Results and Discussions . . . . . . . . . . . . . . 81

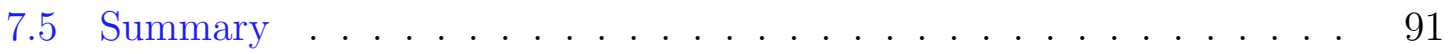

8 GAIT CYCLE EVENT-SPECIFIC SPECTRAL FEATURES 93

8.1 Motivation . . . . . . . . . . . . . . . . . . . . . 93

8.2 Extraction of Gait Phases . . . . . . . . . . . . . . . . . . 94

8.3 Mean Magnitude Spectral Density . . . . . . . . . . . . . . . . 96

8.4 Relationships of Clinical Data to Spectral Features _ . . . . . . . 97

8.4.1 Statistical Analysis . . . . . . . . . . . . . . . . . 97

8.4.2 Results and Discussions _. . . . . . . . . . . . . . 98

8.5 Summary . . . . . . . . . . . . . . . . . . . . . . 101

9 SUMMARY AND CONCLUSION 104

$\begin{array}{ll}\text { Appendix } & 111\end{array}$

$\begin{array}{ll}\text { Bibliography } & 139\end{array}$ 


\section{List of Tables}

1.1 Gait features . . . . . . . . . . . . . . . . . . 9

3.1 Summary of clinical data categories . . . . . . . . . . . . 38

4.1 Demographics of controls and MS subjects . . . . . . . . . 45

6.1 Spearman correlation coefficient between clinical data and the gradient of STSD . . . . . . . . . . . . . . . . . . 63

6.2 The adjusted $R^{2}$ from step-wise LRMs . . . . . . . . . . . 64

6.3 The $\%$ adjusted $R^{2}$ for four aspects of MS disability . . . . . . . 69

7.1 Statistics of the KDE peak . . . . . . . . . . . . . 78

7.2 The adjusted $R^{2}$ from step-wise LRMs $\ldots \ldots \ldots \ldots$

7.3 The $\%$ adjusted $R^{2}$ for four aspects of MS disability . . . . . . 90

8.1 Summary of the spectral features . . . . . . . . . . . . 97

8.2 The $\%$ of variance in the spectral features predicted by the clinical data 99

8.3 The adjusted $R^{2}$ showing the proportion of variance in clinical subscores as explained by the spectral features . . . . . . . . . . . . 103

9.1 Summary of the results . . . . . . . . . . . . . . . 106

9.2 The \% contributions of various aspects of disability in MS to the inertial measures . . . . . . . . . . . . . . . . . 106 


\section{List of Figures}

2.1 IMFs of a gait cycle . . . . . . . . . . . . . . . . 20

2.2 A sinusoid with two sample values interchanged . . . . . . . . . 22

2.3 IMFs of a sinusoid without extrema adjustment . . . . . . . . . 23

2.4 Illustration of extrema removal process . . . . . . . . . . . 28

2.5 IMFs of gait cycles with and without extrema removal . . . . . . 29

2.6 Number of IMFs generated using original and modified EMD . . . . . 30

2.7 The Hilbert spectra of IMFs of a gait cycle . . . . . . . . . . . . . . 32

2.8 HHT block diagram . . . . . . . . . . . . . . . . . . 35

4.1 Sensing axis relationships of inertial node worn on ankle . . . . . 44

4.2 Human gait phases . . . . . . . . . . . . . . . . . . . 46

4.3 Gait signal and its components . . . . . . . . . . . . . 47

4.4 Gait signal and its components . . . . . . . . . . . . . 48

6.1 STSD for study groups . . . . . . . . . . . . . . . . 56

6.2 STSD for minute $1 \ldots \ldots \ldots \ldots \ldots \ldots$

6.3 STSD for minute $2 \ldots \ldots \ldots \ldots \ldots \ldots \ldots$

6.4 STSD for minute $3 \ldots \ldots \ldots \ldots \ldots$

6.5 STSD for minute $4 \ldots \ldots \ldots \ldots \ldots \ldots$

6.6 STSD for minute $5 \ldots \ldots \ldots \ldots \ldots \ldots$

6.7 STSD for minute $6 \ldots \ldots \ldots \ldots \ldots \ldots$

6.8 Gradient of STSD . . . . . . . . . . . . . . . . . . . . . 61

6.9 Distance covered during the 6MWT by study groups $\ldots \ldots \ldots$. . . 62

6.10 Relationships of FSS components and EDSS to stride time variability 65

6.11 Relationships of MFIS components and EDSS to stride time variability 66

6.12 Relationship of total MFIS to stride time variability . . . . . . . 67

6.13 Relationships of MSWS components to stride time variability . . . . . 67

6.14 Relationship of PDDS to stride time variability . . . . . . . . 68

6.15 Relationship of mobility and spasticity to stride time variability . . . 68

$6.16 \%$ of variance explained by the four aspects of MS disability . . . . 69

7.1 6MWT gait data and its KDE . . . . . . . . . . . . 74

7.2 Typical KDEs of gait time series for various study groups . . . . . . 75

7.3 KDE peaks for various study groups . . . . . . . . 76 
7.4 Atypical KDEs for controls and MS subjects . . . . . . . . . 77

7.5 The KDE for each minute of the 6MWT gait data for an MS subject 78

7.6 Relative 6MWT distances covered by the study groups and corresponding $\mathrm{KDE}$ peaks . . . . . . . . . . . . . . . . . . . . 79

7.7 Relationship of EDSS to KDE peak . . . . . . . . . . . . . . . 81

7.8 Relationships of FSS components to KDE peak . . . . . . . . . 82

7.9 Relationship of PDDS to KDE peak . . . . . . . . . . . . 83

7.10 Relationships of MSWS components to KDE peak . . . . . . . . . 83

7.11 Relationships of performance scales to KDE peak . . . . . . . . 84

7.12 Relationships of IADLs to KDE peak . . . . . . . . . . . 85

7.13 Relationships of SF36 components (Q4 b, c, e, f) to KDE peak . . . 86

7.14 Relationships of SF36 components (Q4 g-j, total score) to KDE peak 87

7.15 Relationships of MFIS components (Q7, 10, 17, 20) to KDE peak . . 88

7.16 Relationships of MFIS components (Q7 and total score) to KDE peak 89

$7.17 \%$ of variance explained by the four aspects of MS disability . . . . 90

8.1 A gait cycle re-sampled to 300 samples . . . . . . . . . . . . 95

9.1 Summary of the results . . . . . . . . . . . . . . . 108

9.2 The $\%$ contributions of MS disabilities to the inertial measures . . . . 108 


\section{Frequently Used Abbreviations}

$\begin{array}{ll}\text { 6MWT } & \text { Six-minute walk test } \\ \text { AIC } & \text { Akaike information criterion } \\ \text { BP } & \text { Blood pressure } \\ \text { BSN } & \text { Body sensor network } \\ \text { CNS } & \text { Central nervous system } \\ \text { DTW } & \text { Dynamic time warping } \\ \text { EDSS } & \text { Expanded disability status scale } \\ \text { EMD } & \text { Empirical mode decomposition } \\ \text { FSS } & \text { Functional systems scores } \\ \text { HHT } & \text { Hilbert-Huang transform } \\ \text { IADL } & \text { Instrumental activities of daily living } \\ \text { LRM } & \text { Linear regression model } \\ \text { LLE } & \text { Left lower extremity } \\ \text { LUE } & \text { Left upper extremity } \\ \text { HS } & \text { Heel strike } \\ \text { HS } & \text { Hilbert spectrum } \\ \text { IMF } & \text { Intrinsic mode function } \\ \text { KDE } & \text { Kernel density estimate } \\ \text { MSD } & \text { Magnitude spectral density } \\ \text { MMSD } & \text { Mean magnitude spectral density } \\ \text { MHI } & \text { Mental health inventory } \\ \text { MS } & \text { Mid-swing } \\ \text { MFIS } & \text { Modified fatigue impact scale } \\ \text { MS } & \text { Multiple sclerosis } \\ \text { MSIS } & \text { Multiple sclerosis impact scale } \\ \text { MSWS } & \text { Multiple sclerosis walking scale } \\ \text { OBW } & \text { Occupied bandwidth } \\ \text { PDDS } & \text { Patient-determined disease steps } \\ \text { PRO } & \text { Patient-reported outcome } \\ \text { PS } & \text { Performance scales } \\ \text { PNS } & \text { Peripheral nervous system } \\ \text { RLE } & \text { Right lower extremity } \\ \text { RUE } & \text { Right upper extremity } \\ \text { SF36 } & \text { Health status questionnaire } \\ \text { SD } & \text { Standard deviation } \\ \text { STSD } & \text { Stride time standard deviation } \\ \text { TEMPO } & \text { Technology enabled medical precision observation } \\ \text { TO } & \text { Toe-off } \\ \text { ZC } & \text { Zero-crossing } \\ & \end{array}$




\section{List of Symbols}

$\begin{array}{ll}\alpha & \text { Significance level } \\ \Delta_{6 M W T} & \text { Gradient of the stride time standard deviation } \\ \mu & \text { Mean } \\ \rho & \text { The KDE peak } \\ \sigma_{s t} & \text { Stride time standard deviation } \\ \tau & \text { Dummy variable } \\ \theta(t) & \text { Instantaneous phase } \\ a(t) & \text { The envelope of an analytic signal } \\ B(t) & \text { The gait signal } \\ \bar{B} & \text { Average stride time } \\ c(t) & \text { An IMF } \\ c(t) & \text { The Hilbert transform of an IMF } \\ C(t) & \text { Gait cycle } \\ f & \text { Frequency } \\ F_{s} & \text { Sampling frequency } \\ h & \text { The marginal spectrum } \\ h_{K} & \text { Kernel function bandwidth } \\ H & \text { The Hilbert spectrum } \\ K & \text { Kernel function } \\ L(t) & \text { The lower envelope of a signal } \\ m x & \text { Set of the locations of the maxima } \\ m n & \text { Set of the locations of the minima } \\ N & \text { Number of samples } \\ p & \text { p-value } \\ P & \text { Power } \\ R & \text { The adjusted R squared value } \\ s(t) & \text { An arbitrary signal } \\ r(t) & \text { Residue of EMD } \\ r_{p} & \text { Pearson's correlation coefficient } \\ r_{s} & \text { Spearman correlation coefficient } \\ t & \text { Time } \\ U(t) & \text { The upper envelope of a signal } \\ X & \text { Arbitrary random variable } \\ x(t) & \text { Analytic signal } \\ & \end{array}$





\section{Chapter 1}

\section{INTRODUCTION}

Walking is a sequence of repeated gestures known as gait cycles. For these gestures to take place, the nervous system and musculo-skeletal system must function and coordinate properly to generate a sequence of events to complete the process of walking. The events are mainly an activation of walk commands in the central nervous system (CNS) and their passage to the peripheral nervous system (PNS) followed by the development of tension by alternately contracting and relaxing muscles and, finally displacement and generation of ground reaction forces [1]. Thus gait dynamics carries substantial information about human health and has been proven to measure not only physical and functional impairment but also neurological integrity and functions. Some applications of gait analysis include - (i) medical research applications and healthcare management, such as fall prevention and risk assessment, rehabilitation engineering, assessment of orthopedic surgery, prosthetic design, elderly in-home monitoring, diagnosing dementia and cognitive disorders, (ii) sports medicine and bio-mechanics for athletic training and performance optimization, and (iii) recognition bio-metric using a combination of spatial-temporal and kinematic features to identify persons. 
Multiple sclerosis (MS) is a neurological disorder that destroys axons [2], the mechanism of communication between the brain and other parts of the body, resulting in neurological, physical, and functional deterioration [3]. Gait dysfunction is an almost ubiquitous symptom even in the early stages of the disease, but one with varied etiology, and could be the result of MS-induced fatigue, balance problem, weakness, sensory loss, or other clinical symptoms. The increased incidence and severity of gait dysfunction is found in tandem to increased disease severity [3]. Loss of gait function results in significant reduction in functional independence and quality of life in MS [4]. Thus assessment of walking capacity over the course of disease progression is an integral part of MS evaluations.

The most commonly used diagnosis test for MS is the McDonald criterion [5]. The physician's judgement in interpreting the outcomes of neurological exams, medical history, and medical imaging results, if used, is a crucial part of the diagnosis [6]. After diagnosis, persons with MS are categorized into disability levels such as mild, moderate, severe, etc., depending on the outcomes of functional system scores [7]. In addition, objective timed-walking tests such as six-minute walk test (6MWT) $[8,9]$ and subjective questionnaires $[10,11]$ are used to assess the impact of MS on specific functional, physical, or cognitive functions.

Each type of assessment has important limitations, such as being qualitative, prone to floor and ceiling effects, dependent on physicians' judgment in interpreting outcomes of imaging and clinical symptoms, affected by the differences among individuals regarding the notion of disability or improvements, time-consuming, being imprecise (over-/under-reporting, missing data being difficult to interpolate), having low variance across ratings, limited sensitivity to subtle changes in functions, affected by the response shifts [12] due to changes within individuals regarding health standards, and being burdensome for the persons with MS. Furthermore, severity 
and disability assessments are neither conclusive nor based on a simple yes or no criterion [6]. Lack of clinically meaningful objective features could lead to confusing findings and discrepancies between expected and observed indicators, and thus making it difficult to make an accurate prognosis.

The high-precision, non-invasive, and continuous data collection capabilities of inertial body sensor networks (BSNs) has led to great interest in finding objective markers of neurological disorders and disease progression, including MS. Previous works have demonstrated the potential of using inertial gait features for quantitative mobility assessments in persons with MS. The features studied include, but are not limited to, energy expenditure estimator [13], causality index [14], stance to swing ratio [15], double support [15], Lyapunov exponent [16], and warp scores [17], each with a certain separability performance. These works primarily focus on finding gait variables that improve separability performance to distinguish between controls and MS subjects with or without reference to MS disability levels, assigned using an expanded disability status scale (EDSS) [7], and often, the relationships between inertial gait variables and clinical data are not explored. With our research, we plan to augment MS research taking into account a few of the currently open areas, as described in the next section.

\section{$1.1 \quad$ Research Motivation}

The importance and motivation to do this research are given below.

1. People with MS represent a heterogeneous group with a broad spectrum of physiological, functional, and psychological symptoms that vary among individuals and within individuals over time [18]. Thus, it is important to analyze both within and across group differences. Individuals within a certain disability group might 
have statistically different gait parameters and gait analysis of individuals initially categorized into different disability groups could produce similar gait variables depending on the specific impact of MS on their functions. For example, limitations in doing household chores could be due to the impact of MS on physical functions for one MS subject and on cognitive functions for another.

2. There is a limited literature on the exploration of the relationships between inertial measures and clinical data. Despite having low variance in ratings, clinical measures are easier to be interpreted by the physicians, so the relationships of clinical data to inertial measures should be explored for the following reasons:

- To validate inertial features as clinically meaningful markers

- To give physiological meanings to the gait features and make them easily interpretable and more accessible by the doctors

- To tailor subjective questionnaires based on individual needs

- Wearable BSNs technology is now advanced enough to support high-precision, continuous, minimally invasive data collection, without human intervention. Thus, once an inertial feature is validated as a marker of the disease, it could be employed on inertial nodes enabling doctors/physicians to monitor the disease and its progression, continuously, objectively, and remotely. The longitudinal data could be leveraged to evaluate the efficacy of therapies, predict the course of the disease, and self-management by the persons with MS.

- Features might have high variance across subjects but show no clear relationships with any subjective assessments or clinical symptoms. Identification of such features is important, since it might be an indication of information that 
is missing from current subjective assessments, and needs to be captured by new/alternate means.

The relationships of inertial measures to clinical data would allow patient's education and engagement, whereby people can keep a track of their gait features, besides other vital signs, relate changes in their gait variables to their lifestyles or therapies, and take informed and active decisions regarding the ongoing treatments, thus opening directions for out-of-clinic monitoring and management of symptoms.

3. While absolute assessments are important in order to understand population characteristics, examination of variations in an individual's baseline over time is useful to remove inter-patient variability. The gait parameters should be computed over an interval of time, when possible, and the amount of change in them should be observed and tracked with the changes in clinical measurements. For example, when using timed-walking tests [8] to assess the gait quality, not only the standard deviation SD of the stride time (time elapsed between two consecutive heel strikes of the same foot), $\sigma_{s t}$, but also its variability over time should be examined. Small but variable $\sigma_{s t}$ could be an indication of balance problems or other aspects of walking impairment.

4. Computation of most of the commonly used gait variables such as stride time standard deviation (STSD), dynamic time warping (DTW) scores, stride length, gait phase, depend on accurate identification of gait cycles, which is a computationally expensive and noise-sensitive process. Thus gait features that don't require segmentation of gait data into individual cycles and could be derived from a subset of the data would be beneficial from the standpoints of storage and processing requirements. Such features could be adapted and implemented on resource-constrained 
inertial nodes for longitudinal gait monitoring.

5. There is an opportunity to leverage gait phases, such as stance and swing, as they may provide additional information regarding the variable and precise impact of the disease on these events. Component-specific features and their relationships to the clinical data can be used as predictors of balance, stability, fall risk, etc. Thus, an event-specific study of gait variables is needed.

\subsection{Societal Motivation}

According to [6], MS has a median estimated incidence of 2.5 in every 100,000 persons and 1.3 million diagnoses, around the globe, with twice as many women diagnosed with MS as men. The loss of motor functions in persons with MS, including young adults, results in significant reduction in functional independence and quality of life $[4,6]$. Since persons with MS need help with daily activities and emotional support, MS impacts the family members as well. The amount of physical and emotional burden increases as the disease progresses. Persons with MS eventually leave the workforce. Loss of personal self-sufficiency then creates a financial burden on society.

Current clinical practices are subjective and rely on physician's judgement and reports from persons with MS, thus could be time-consuming, infrequent, and imprecise. Accurate and objective mobility assessments are important not only for successful interventions, but also for monitoring the disease progression and evaluating efficacy of the therapies. MS is an unpredictable, chronic disorder. For maximized wellness, patients' education and engagement are important to help them make informed decisions, re-gain their independence, and improve the quality of life [6]. Objective measures are accurate but must be tied to physiological processes, when possible, 
by interpreting them in clinical context, thereby making them meaningful and easily understandable by the doctors and persons with MS.

The advancements in signal processing and machine learning techniques can be used for characterizing the gait impairment accurately and quantitatively thus improving healthcare and reducing healthcare costs. In addition, the use of non-invasive, wearable inertial sensors will enable continuous monitoring of disease course.

\subsection{Broader Impacts}

Our test features can be adopted in relevant domains such as evaluating the effectiveness of treatments and therapies, fall prediction, guiding recovery and rehabilitation, gait monitoring in elderly, and general health and wellness monitoring. They can also be employed in other neurological disorders in which gait deterioration and disability are common problems. Examples of neurologic disorders whose gait characteristics have been examined by researchers using inertial sensors include Friedreich's ataxia [19], Huntington's disease [20], Parkinson's disease [21], Alzheimer's disease [22], normal pressure hydrocephalus (NPH) [23], and stroke [24].

\subsection{Our Contribution}

We extract inertial features from gait of MS subjects, collected using ankle-worn BSN nodes, and study the relationships of gait features to the subjective assessments to establish our test features as clinically relevant and meaningful objective anchors of the disease. We also determine the physiological significance of our feature set related to four crucial aspects of MS-induced impairment, namely, weakness, balance, physical fatigue, and cognitive/concentration. The 6MWT, a standardized walking test used to assess dynamic motor fatigue [8], was used as the protocol to collect the 
inertial data. Two types of gait features are investigated:

1. Temporal features. These features represent temporal aspects of the gait. Following two temporal features are studied:

- The STSD and its gradient over six-minutes. They measure the variations in the stride time as subjects perform the 6MWT test. Higher values reflect poor walk quality, which could be either due to the impact of MS on gait or a fatigue-induced effect.

- The normal kernel density estimate (KDE). The KDE is obtained using the $6 \mathrm{MWT}$ gait time series. The relationships to clinical data show that the value of KDE peak above a threshold indicates balance and weakness issues in MS.

2. Spectral features. The magnitude spectral density, corresponding to important events comprising a gait cycle, is computed and seven spectral features are extracted. The relationships of spectral features to the clinical data show that different phases of gait carry important information about the state of the disease.

To maximize the benefits of data analysis, the signal processing techniques employed should be well-suited to the data-set being investigated. Since gait data are non-stationary [25], spectral analysis using a transform based on a fixed basis and/or stationary assumption will only give an average state of the gait and not reflect the changes in its behavior occurring during the observation interval, if any. Thus the transform basis should be local and adaptive to the variations in the data for it to be able of analyzing the non-stationary data [26]. The Hilbert-Huang transform (HHT) [26] basis satisfies these conditions, making it a suitable candidate for gait analysis, and thus is used in this work. 
The HHT expands a non-stationary time series using an adaptive data-driven basis as a sum of zero-mean components and then applies the Hilbert transform to each of these components to yield an spectrum based on instantaneous amplitude and frequency, unlike the global distribution formulated by the Fourier analysis. The final three-dimensional amplitude-time-frequency representation is commonly known as the Hilbert spectrum [26]. The process to generate the basis functions is, however, extremely sensitive to noise and small changes in the underlying data. To make the analysis step of HHT stable, we modify the decomposition algorithm and use modified version for extracting the spectral features. Table 1.1 gives a summary of the feature space.

Table 1.1: Gait features

\begin{tabular}{l|l|l}
\hline \hline Feature & Input & Output \\
\hline \hline Stride time & $\begin{array}{l}\text { Set of identified gait } \\
\text { cycles }\end{array}$ & $\begin{array}{l}\text { a. STSD } \\
\text { b. Gradient of the STSD from minute 1 to } \\
\text { minute 6 }\end{array}$ \\
\hline $\begin{array}{l}\text { Kernel density esti- } \\
\text { mate (KDE) }\end{array}$ & Segment of gait data & KDE peak \\
\hline $\begin{array}{l}\text { Magnitude spectral } \\
\text { density }\end{array}$ & $\begin{array}{l}\text { 3D HHT spectra of a } \\
\text { set of gait cycles }\end{array}$ & $\begin{array}{l}\text { a. Mean magnitude spectral density } \\
\text { b. Corresponding feature vector }\end{array}$ \\
\hline \hline
\end{tabular}

We found that the typical ranges of gait variables corresponding to EDSS-based disability groups overlap with each other. In fact, the gait variables for subjects in the lower disability categories overlap with those of control subjects. Thus the gait differences among various EDSS categories might not always be seen and observing persons with MS in different disability groups having statistically similar gait variables is likely. On the other hand, although not precise, PROs represent the ground-truth regarding the functional and cognitive state of persons with MS. So, we also study the relationships between the inertial features and PROs to look for gait differences among subjects or within individuals over time, and possibly identify the clusters of 
gait variables without restricting to disability groups, as defined by EDSS. Lastly, to unearth the physiological significance of the inertial features, we employ regression analysis [27, ch. 3], a data mining [28] technique, to generate models predicting the gait variables using clinical assessments. The interpretations of inertial measures from a clinical viewpoint are important for successfully employing new gait features along with traditional speed based metrics as disease markers. By exploring the relationships of our test measures with the clinical data, we support the clinical and research application of inertial features to MS and other pathologies affecting gait. Data from comparable control subjects are also used to identify distinct and overlapping features. All analyses were performed using MATLAB R2016aß).

We believe that with our research, we are augmenting existing information, ongoing research, and currently-used speed and distance-based clinical assessments with new, high-precision objective anchors to fulfill the requirements unmet by the current measures. The relationships of inertial measures to clinical data can be used to improve the precision of subjective questionnaires by customizing them based on individual needs. The measures studied in this work can be used as complimentary anchors, alongside traditionally used gait speed, to advance precise and objective analysis of MS-associated walking disability and their physiologic significance indicates their clinical utility. Using our test features, we hope to better characterize and differentiate features within MS cohorts and between MS and control subjects. Knowledge of changes in these features over time will improve our capacity to longitudinally and continuously monitor the disease progression, evaluate the effectiveness of the treatment, and possibly predict the future state of the disease. 


\subsection{Literature Review}

Inertial measures for research applications and improved healthcare:

Advancements in signal processing and machine learning tools as well as the emergence of BSN has given researchers and clinicians opportunities to employ new and robust inertial measures for a better understanding of the mechanisms of human movement disorders and monitoring of neurological diseases. Such measures are also shown to distinguish subjects clinically diagnosed with a particular disorder from control subjects, each with a certain classification accuracy and separability performance. Results in [29] demonstrate various methods to identify Parkinson's disease tremor using a waist-worn accelerometer node. In [19], inertial data are used to identify Friedreich's ataxia subjects from controls. Sejdic et al. [21] use time and frequency based features to separate Parkinson's and control subjects. Shanshan et al. [30] use inertial measurements to show that ankle-foot orthoses modify heel contact. The authors of [31] investigate the effectiveness of using training sessions based on overground and treadmill walking to improve walking performance after stroke. The potential of using high-precision inertial sensors to estimate relevant gait parameters is shown in [32]. The authors of [33] use a discrete wavelet decomposition of acceleration signals from an ear-worn sensor for assessing gait impairment for continuous monitoring of recovery following knee-replacement surgery. Shrinivasan et al. [23] demonstrate using simple inertial measures such as stance and swing periods, stance to swing ratio, double support to help aid the diagnosis of NPH in a minimally invasive fashion instead of traditional high volume lumbar puncture followed by the evaluation of clinical response. The authors of [34] use inertial data to analyze gait differences between individuals with post-stroke hemiparesis and controls at matched speeds using treadmill walking. Barth et al. [35] show the feasibility of using body sen- 
sors for long-term, high-fidelity gait analysis in healthcare and research applications. The authors of [36] propose linear dynamical modeling for robust clustering of a set of human actions using inertial sensors data. In [16], it is shown that the Lyapunov exponent can be used to assess gait stability with a better separability performance and improved differential diagnosis. The authors of [37] introduce a Mobility Lab to assess gait impairment in neurological disorders. Li et al. [38] use an ear-worn sensor to study gait pattern detection algorithms.

Six-minute walk test (6MWT): The 6MWT has been used in a variety of applications. Garber and Friedman use 6MWT to study the relationship between fatigue and physical function in Parkinson's disease [39]. Goldman et al. assess the correlation of $6 \mathrm{MWT}$ to subjective measures of fatigue, ambulation, and physical function [8]. Savci et al. study the factors that contribute to functional capacity in MS subjects [40]. The authors show that MS subjects cover shorter distance during a 6MWT compared to control subjects due to limited daily activity, fatigue, and resting heart rate. The authors of [41] investigate the link between perceived impact of fatigue on functioning (physical, cognitive and psychosocial) and exertion dyspnea and/or deteriorated cardiorespiratory function during walk for people with mild disability with normal cardiorespiratory response. The results show that the 6MWT distance correlates significantly to a disability score but not to subjective fatigue. In addition, [31, 42-44] have utilized the test to evaluate the benefits of rehabilitation following stroke.

Inertial measures for MS: Zwibel [4] studies the contribution of symptoms such as walking impairment, spasticity, fatigue, depression, and pain, to a patient's quality of life and economic burden of MS. Gong et al. [14] demonstrate that the causality index can be used to study the strength of interaction between body parts to distinguish MS subjects from controls with a better separability than other methods 
available in literature. The authors of [17] show the capability of DTW and warp scores to detect changes in gait dynamics introduced by the 6MWT and validate them as clinical measures alongside gait speed to quantify fatigue-related gait deterioration. Givon et al. characterize MS gait parameters and correlate them with subjects' neurological disability [45]. Martin et al. study balance and gait impairment in MS patients with mild pyramidal signs, with no pyramidal signs, and controls [46]. In [47], Gong et al. determine the correlations of multiple sclerosis walking scale (MSWS) [48] and other clinical measures to body sensor measurements. The authors of [49] discuss the clinical significance of DTW and warp scores to MS symptoms specific to gait impairment. The authors of [50] measure several gait parameters to identify various MS disability groups and control subjects and show the relationships between those parameters and MSWS scores. Crenshaw et al. use reflective markers and a motion analysis video system to study the gait variability in MS subjects and conclude that there was more variability in kinematic measures for MS subjects who also reported more fatigue and walked slower compared to controls [51]. Bourke et al. [13] show that energy expenditure estimation can be improved by combining it with accelerometry and heart-rate measurements. Motl et al. validate accelerometry data, based on its correlations with 6MWT distance and oxygen cost of walking, as objective markers of walking limitations in MS [52]. Sandmann et al. [53] use sensors to capture biomarkers as predictors of day quality of persons with MS. Socie et al. [54] use a waist-worn ActiGraph to assess ambulation in persons with MS. The authors of [55] show that inertial data can be used to capture the variability of the gait features in MS. Huisinga et al. [56] use accelerometry signals to determine linear and nonlinear features as measures of gait variability in persons with MS.

MS clinical research: To reduce the burden on patients, [57] develop and validate a Bayesian reasoning and domain-specific based adaptive symptom reporting 
system to reduce the number of questions required for accurate disability assessment (75\% reduction associated with $4.45 \%$ clinically relevant estimation error). Hausdorff [58] emphasizes the importance of observing the stride-to-stride fluctuations for identification of elderly fallers with higher sensitivity compared to traditional measures such as average gait speed. The authors of [59] evaluate bladder dysfunction in MS and discuss strategies for disturbances management. Alaqtash et al. [60] develop a method for an automated gait classification for analysis and classification of pathological gait patterns using ground reaction forces data. Nowaczyk and Cierpia [18] identify three different psychological profiles of MS patients based on resource conservation using a k-means clustering algorithm and propose that the diverse dynamics of the functioning of MS patients indicated by these profiles should be taken into account for improved multidisciplinary rehabilitation.

Data mining in bioinformatics and biomedical engineering: The authors of [61] discuss the opportunities and challenges for data mining research in emerging domains including biomedical engineering. Kropp and Caulfield [62] review the impact of data mining in bioinformatics on biological research. The authors of [63, 64] show the potential of using data mining to increase the understanding of Parkinson's disease. Bonato et al. [63] use wearable sensors' data to identify patterns as well as severity of motor disorders in Parkinson's disease. The authors suggest that the approach can be generalized to similar diseases affecting motor functions. Ramani and Sivagami [64] conduct a survey of data mining techniques for the classification of persons with Parkinson's disease from healthy subjects. Bejarano et al. [65] assess the diagnostic accuracy of clinical variables, including expanded disability status scale (EDSS), using various computational classifiers and employ data mining for feature selections, for predicting the MS course. Li and Schwartz [12] use a partitioning tree analysis technique to identify response shift patterns in persons with MS. Other ap- 
plications of data mining in bioinformatics include probe selection for gene-expression arrays [66], plant genotype discrimination [67], experiments with automatic cancer diagnosis [68], protein annotation [69], and identification and prediction of drug-induced nausea [70].

\subsection{Dissertation Outline}

The dissertation is organized as follows. The overview of the Hilbert-Huang transform and our signal processing contribution to it are presented in Chapter 2. Chapter 3 addresses the importance of investigating the relationships between clinical measures and inertial features. It also elaborates on various types of clinical data studied in this work. Chapter 4 describes the study procedures, the data collection BSN platform, the gait terminologies, and all related signal processing aspects. Chapter 5 describes the two-step statistical analysis performed used in this work to determine the physiological significance of our inertial features.

The technical contributions and the main results of the dissertation are arranged into Chapters 6 to 8. Chapter 6 studies the first temporal feature, namely, the gradient of the stride time standard deviation. Chapter 7 discusses the second temporal feature, namely, the kernel density estimate of the gait data and also studies the relationships of clinical data to these estimates. Chapter 8 investigates the importance of the phase-specific spectral densities wherein spectra are generated corresponding to different phase of a gait cycle.

Chapter 9 summarizes the whole dissertation and concludes the results. Lastly, the sample questionnaires used to collect subjective data for clinical assessments are given at the end of the dissertation in the appendix. 


\section{Chapter 2}

\section{HILBERT-HUANG \\ TRANSFORM}

The Hilbert-Huang transform (HHT) [26] is a spectral analysis technique. The basis function of a spectral tranform should be suited to the data being investigated to reflect the changes in the data occurring during the observation interval, if any, instead of giving an average state of the underlying process. The transform basis of the HHT satisfies the crucial requirements needed to accommodate the non-stationary data, such as gait [25], and is, thus, used in this work for analyzing the frequency domain characteristics of the gait data. However, the algorithm used to generate the basis functions is extremely sensitive to noise and minor fluctuations in the data. So, we modify the original algorithm and use the modified version for decomposing the gait data [71]. This chapter gives an overview of the HHT and our modification. The modified algorithm was published in [71]. 


\section{$2.1 \quad$ Introduction}

The Hilbert-Huang transform (HHT) is a two-step spectral analysis technique which is used to study non-stationary data. In the first step, the HHT uses an algorithm, known as empirical mode decomposition (EMD), to decompose the data into a set of basis functions and then applies the Hilbert transform to these functions to yield an instantaneous amplitude-time-frequency distribution, known as the Hilbert spectrum [26]. In this chapter, the details of these two steps are given. The original EMD algorithm is sensitive to small changes in the signal. In order to make EMD stable against small variations in the data, we present an improvement of the traditional algorithm, known as selective extrema analysis.

\subsection{Background}

Data analysis is essential in research and clinical applications, and techniques used should be well-suited to the data-set being investigated. Gait data are nonstationary [72]. Such data have no time scale and their analysis require the transform basis function to be local and adaptive for the following two reasons to properly capture their properties [26]:

1. Locality is needed so that events embedded in the signal can be identified by their occurrence times in the absence of a time scale. The first requirement can be fulfilled by using a basis that is allowed to have variable amplitude and frequency over time [26].

2. The basis function should be adaptive to the local variations in the data.

Results from spectral transforms based on a fixed, a priori basis and stationary assumption gives only an average state of the phenomenon and does not reflect the 
changes in the nature of the data occurring during the observation interval, if any.

Fourier and wavelet are the two most common used spectral analysis techniques. However, both of these have shortcomings in the context of non-stationary data analysis [26]. The Fourier transform, for example, requires underlying data to be stationary for the resulting spectrum to make physical sense. The Fourier basis is a set of sinusoidal functions, and thus does not depend on the nature of the data. Moreover, since the sinusoidal functions have constant amplitude and frequency, they can construct stationary data only. The wavelet transform, on the other hand, solves the locality problem by letting resultant components having parameters that determine their own scale over time. Adaptivity, however, is still an issue since, similar to the Fourier transform, the decomposition basis should be set a priori [26]. There are also other methods, each with own benefits and limitations, that are designed to modify the global representation of the Fourier analysis in some way [73, 74].

The Hilbert-Huang transform (HHT) $[26,75]$ is a spectral technique whose transform basis fulfills the two requirements crucial to accommodate the non-stationary data. The basis function is adaptive and data-driven, determined by the initial data itself. HHT is comprised of two steps: the first step is to use an empirical mode decomposition (EMD) algorithm to decompose a time series into a set of intrinsic mode functions (IMFs) components and possibly a residue, a function that can not be decomposed into more IMFs. The second step is to apply the Hilbert transform to these IMFs to yield an amplitude-time-frequency distribution, known as the Hilbert spectrum. The final representation is in terms of instantaneous amplitude and frequency rather than the global amplitude and frequency defined by traditional Fourier spectral analysis [26]. 


\subsection{Intrinsic Mode Function}

An IMF is a simple oscillatory mode, as a counterpart to a simple harmonic function, but it can have variable amplitude and frequency along the time axis [26]. Each successive IMF contains lower frequency oscillations than the preceding one.

Throughout this chapter, we use the following notations:

- $s(t)$ is the analyzed signal sampled into $N$ samples thus $\left\{t_{1}, t_{N}\right\}$ are the endpoints.

- $\{1, N\}$ are the indices or locations of the endpoints.

- $m x$ and $m n$ are the sets containing the locations of local maxima and minima of $s(t)$, respectively.

- The extrema are the locations of maxima and minima combined.

- $c_{i}(t)$ refers to the $i$ th IMF.

- If the remainder of the decomposition is sufficiently small or becomes a function from which more IMFs can not be obtained, it is called the residue $r(t)$.

For a function $c(t)$ to be a candidate IMF, it should satisfy two requirements:

1. The size of $m x$ and $m n$ combined and the number of zero-crossings (ZC) must either be equal or differ at most by one (allowed to have exactly one zero between successive extrema). If $|\cdot|$ represents the cardinality of a set, then this condition can be written mathematically as

$$
\operatorname{abs}(|m x|+|m n|-|Z C|) \leq 1
$$


2. At any point, the mean value of the upper envelope (curve connecting the local maxima) and the lower envelope (curve connecting the local minima) is zero (zero local mean).

\subsection{Empirical Mode Decomposition}

The original EMD was proposed as an algorithm in [26]. The EMD method is adaptive and efficient and uses sifting to systematically decompose a time series into one or more IMFs, based on the characteristic time scales present in the time series. The synthesis equation for a signal $s(t)$, decomposed into $n$ IMFs and a residue, is given as

$$
s(t)=\sum_{i=1}^{n} c_{i}(t)+r(t) .
$$

Fig. 2.1 shows typical IMFs of an inertial measurement of a gait cycle.
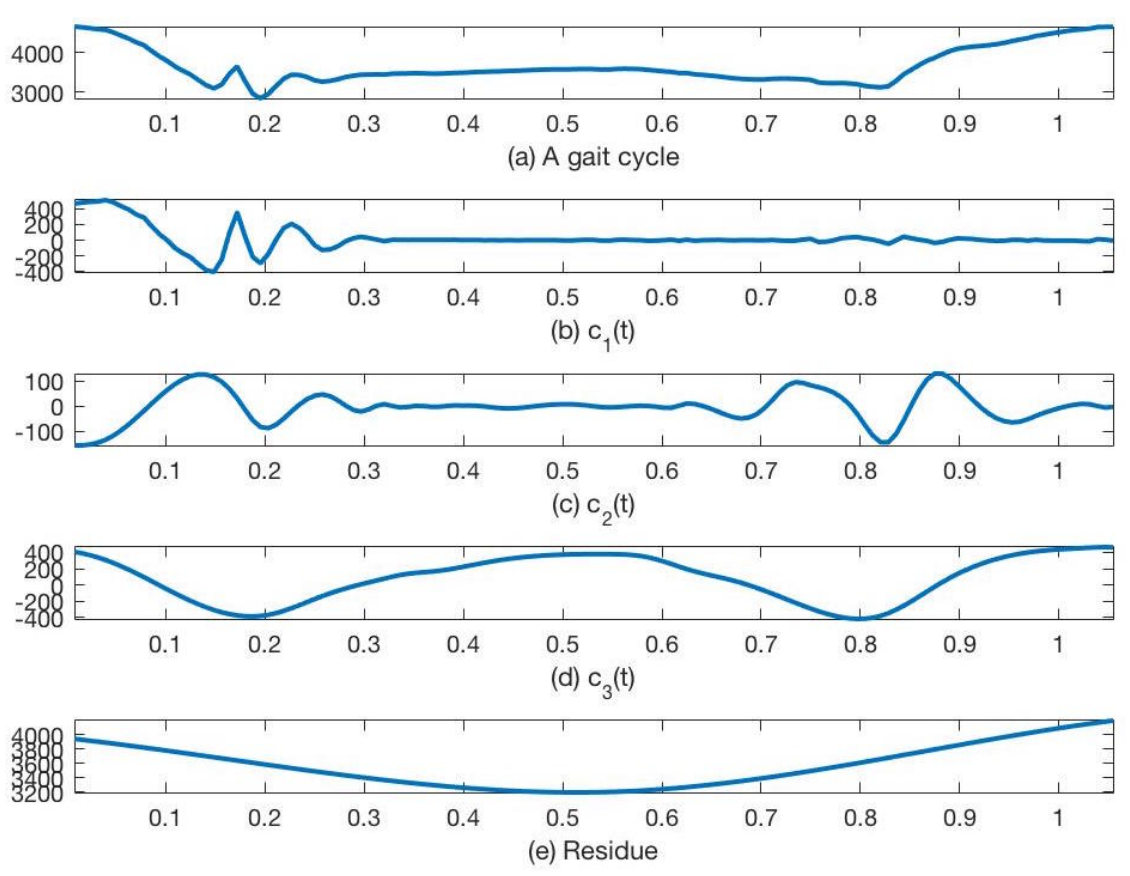

Fig. 2.1: A gait cycle (a) decomposed into three IMF components (b-d) and a residue (e) 
IMFs can have variable amplitude and frequency along the time axis, and thus can be used to expand the non-stationary data.

\subsubsection{Sifting Process and its Instability}

Every step of sifting starts with determining the $m x$ and $m n$ and connecting them using cubic splines to generate the upper and lower envelopes, respectively. It is an iterative process, and the result of successive iterations depend on the results of envelope interpolation in the previous iteration. See [26] for details.

EMD is sensitive to the extrema of the signal and even small changes in the location and number of extrema might make decomposition look different from one obtained in the absence of these changes/noise. The following example illustrates this point. Consider a simple sinusoid

$$
s(t)=\sin (\omega t)
$$

The signal is essentially an IMF as it satisfies the definition of an IMF. Let us interchange two random samples of $s(t)$ to yield an extra hump in the signal, as shown in Fig. 2.2. Even though the difference between the signal amplitude at this pair is negligible, the original EMD generates a completely different decomposition (from the single IMF decomposition $s(t)=c_{1}(t)$ ), as shown in Fig. 2.3.

In cases where a higher frequency noise is superimposed on a lower frequency signal, one or more low-order IMFs can be thrown away to remove noise from the signal and obtain a partial signal reconstruction; but in cases where minor changes do not matter or noise is insignificant and has corrupted only a few signal values, it would be desirable to not generate extra $\operatorname{IMF}(\mathrm{s})$ accounting for small changes in the signal. Instead, our modified sifting algorithm can be used to obtain an approximate 


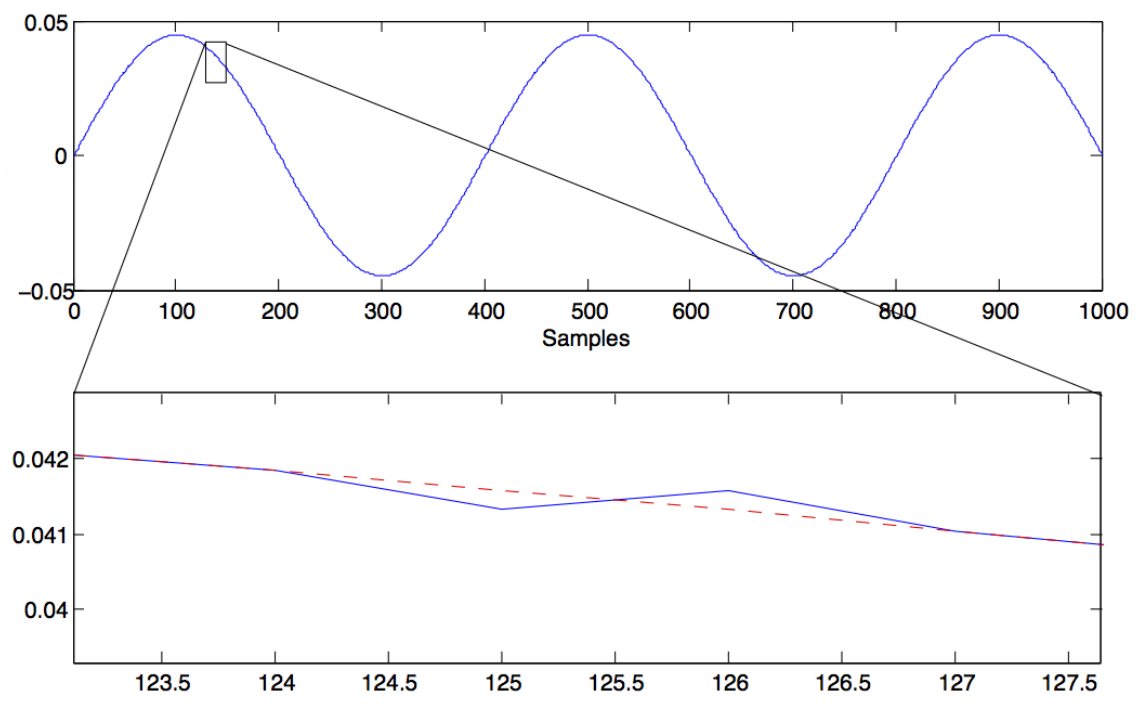

Fig. 2.2: A sinusoid with two sample values interchanged

but stable decomposition with an arbitrarily small decomposition error. We call our modified process selective extrema analysis. With our modified algorithm, these two extrema will be ignored and the shapes of the splines will not be impacted. Thus, unless the application demands to keep all the extrema to highlight this change of values, we can discard this extrema pair and still take $s(t)$ as the one (modified) IMF.

\subsubsection{Selective Extrema Analysis}

Selective extrema analysis uses a subset of the actual extrema obtained from the analyzed signal at every step of sifting. The final decomposition has a number of IMFs no larger than the original decomposition and is different from what we would obtain if the whole set of extrema was used for sifting. The extrema adjustment makes the modified decomposition insensitive to small changes in the signal, in the presence of sampling errors or slowly varying weak noise, and thus helps reduce the number of IMFs a signal is decomposed into. Without extrema adjustment, EMD might decompose two very similar signals into a different number of components and thus different looking IMFs, making it difficult to compare corresponding IMFs (as 


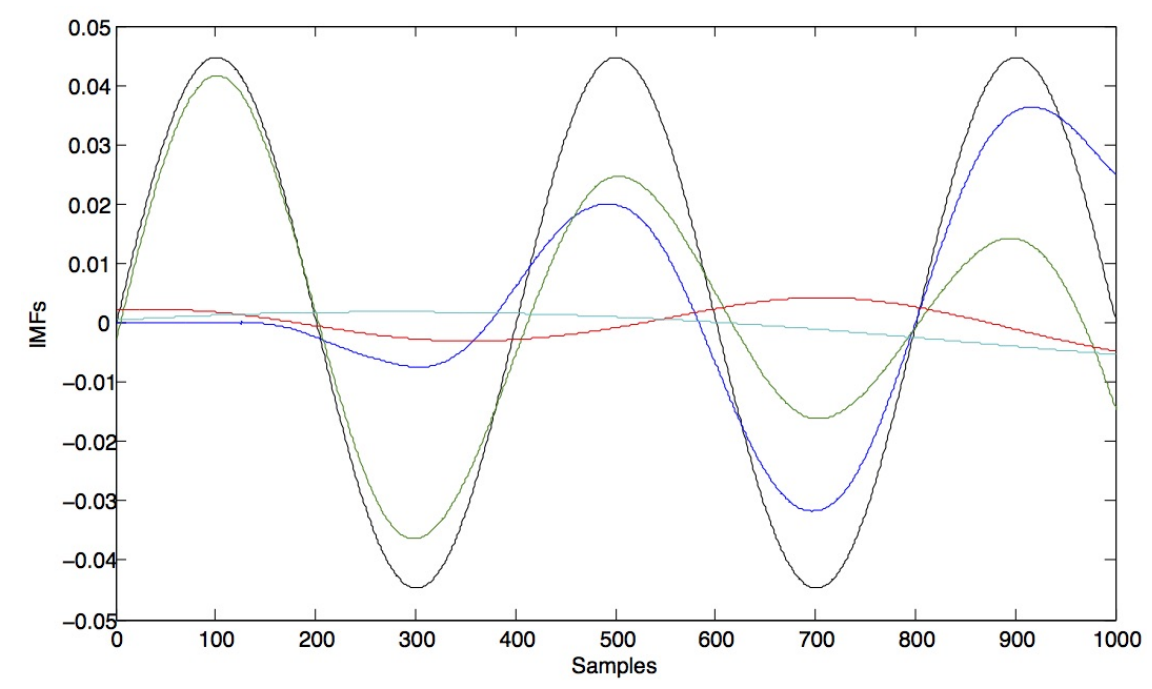

(a)
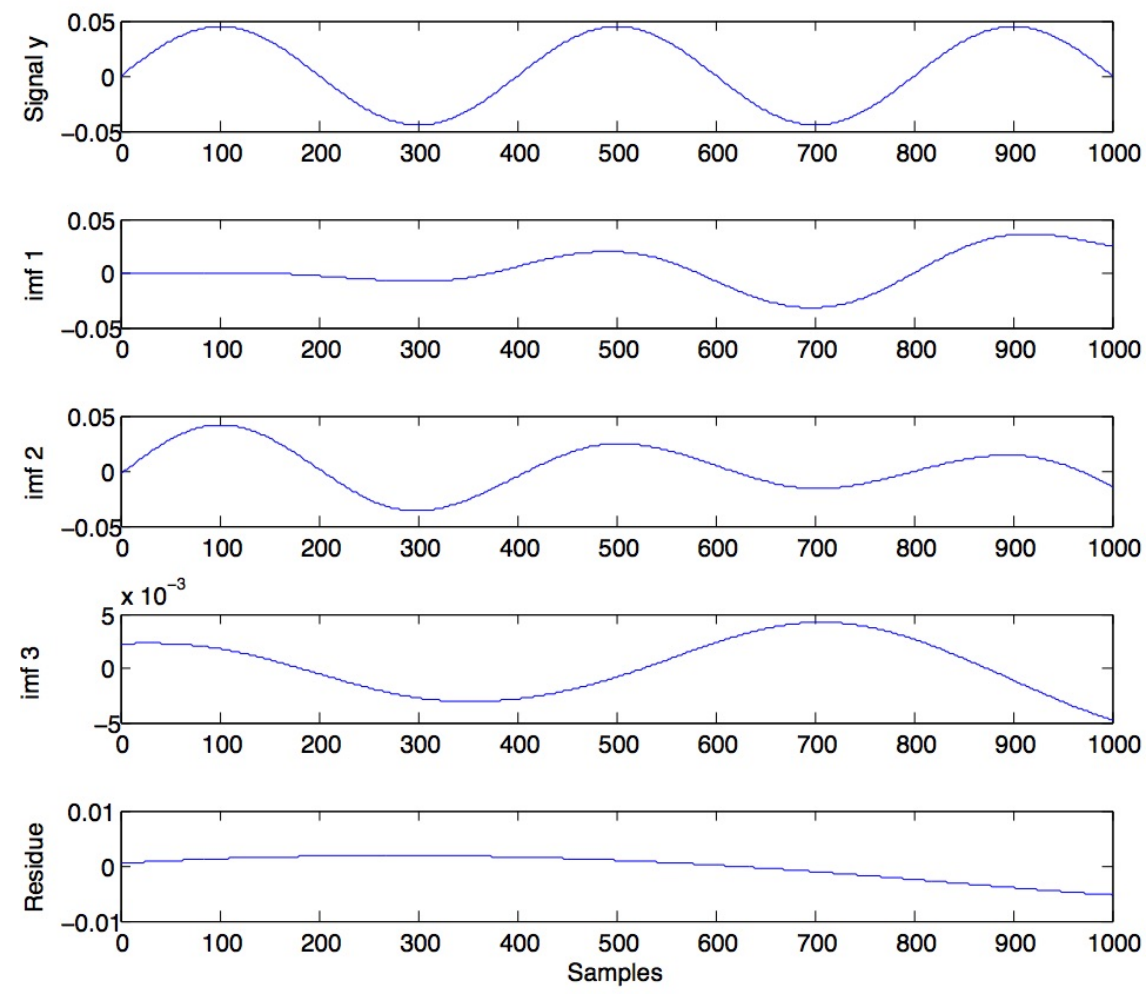

(b)

Fig. 2.3: IMFs of Fig. 2.2 without any extrema adjustment; (a) original signal and all IMFs, (b) individual IMFs identified

they would have different scales) and draw conclusions. Extrema can be adjusted to let EMD yield the same number of modified IMFs for somewhat different signals. 
This makes comparison of IMFs possible and convenient.

We propose to compare the heights of neighboring extrema using an exhaustive search. If the heights are close enough, i.e., if the difference between the heights of neighboring extrema is within a certain (preset or adaptive) threshold, we remove these extrema from the set of candidate extrema used for sifting. Thus the decomposition is different from the original EMD as modified IMFs might not truly satisfy the conditions given in (2.1).

Although the decomposition is changed, we gain two benefits. First, the modified algorithm lets EMD decompose signals into a fewer number of approximate basis functions. Second, in some applications, we need to compare the data before and after processing of some kind. If we get a different number of IMFs from pre- and post-processed data-sets, it is difficult to compare the IMFs. However, if we are able to decompose both data-sets into the same number of (approximate) IMFs, it is then possible to compare corresponding IMFs and their spectra for changes and draw useful conclusions.

Initially, endpoints are neither a part of $m x$ nor $m n$. Let us define a new set $\epsilon$ that contains $m x, m n$, and the endpoints $\{1, N\}$. Since extrema adjustments might need to be done near endpoints, endpoints must be included in the set $\epsilon$ before extrema adjustment. As $m x$ and $m n$ are disjoint, $\epsilon$ can be written as

$$
\epsilon=\{1, \operatorname{sort}(m x \cup m n), N\} .
$$

Here sorting is done in ascending order of time indices, and thus $\epsilon$ contains alternating locations of maxima and minima and is used for extrema adjustment.

We carry out the adjustment in two steps, first working with groups of neighboring extrema triplets and then with groups of neighboring extrema pairs. We always 
throw away extrema in pairs to make sure elements of $\epsilon$ are alternating minimamaxima locations of the signal $s(t)$. Thus triplets look like $\{\max , \min , \max \}$ or $\{\min , \max , \min \}$ and, of course, pairs look like $\{\max , \min \}$ or $\{\min , \max \}$.

1. Pick the triplet which has closest vertical heights (i.e., three neighboring extrema at which the amplitude of the signal deviates the least). Double differentiation can help identify such a triplet, for example. Check if this deviation is within the threshold. If it is not, there is no need to analyze any further triplet, as, obviously in this case, the deviation in signal values at all the other triplets will be larger than the threshold used for extrema adjustment; move to Step II. But if the amplitude of the signal at this triplet deviates within the threshold, we can throw away one of the $\{\min , \max \}$ or $\{\max , \min \}$ pairs in the triplet, whichever has a smaller vertical distance. Note this is a relative comparison and hence, no threshold is needed here. Thus we replace $\epsilon$ with the remaining extrema. We repeat Step I using this pruning process until all sets of triplets have been considered, exhaustively.

2. Using the same sequential exhaustive search of step I, but now with extrema pairs instead of triplets, we eventually remove all unnecessary extrema pairs.

It is possible that extrema adjustment occurs near the edges of the signal, so we include boundaries in $\epsilon$. The algorithm works as follows near the boundaries. In Step I, if a candidate triplet is found for adjustment that includes any of the edges, it keeps the endpoint(s) and throws away the other pair instead. (This won't be a problem as the deviation at this triplet is already within the threshold). In Step II, if it finds an extrema near the boundary such that deviations of signal amplitude at this $\{$ extrema,endpoint $\}$ pair is within the threshold, it discards that extrema (not the endpoint). If we throw away the endpoint(s) and instead keep the other maxima 
or minima near the edges, we generate an extraneous extrema pair when we replicate the signal for boundary adjustment. In the end, we can obtain modified maxima and minima $m x_{1}$ and $m n_{1}$ from the remaining elements in $\epsilon$ for sifting.

\subsubsection{Setting the Threshold}

Any reasonably small threshold works and often helps reduce the number of IMFs. The IMFs defined in (2.1) guarantee a well-behaved Hilbert transform. So, setting a higher threshold and over-adjusting the extrema will not be useful, as it might lead to a large decomposition error, yielding incorrect decomposition and meaningless spectra.

\subsubsection{Mirror Extension for Boundary Condition}

The mirror extension technique of $[76,77]$ is used with the EMD algorithm in order to properly extend interpolation to the edges of the signal. Mirror extension repeats the image of the signal to the left and right and transforms the sets $m x$ and $m n$ into extended sets. Doing mirror extension after extrema analysis is efficient because it avoids the need to perform extrema analysis on extended versions of sets $m x$ and $m n$.

\subsubsection{Stopping Criterion}

The stopping criterion is extremely important in the EMD procedure as it affects the number and the shape of the IMFs. Ideally, the sifting should be stopped when the result of sifting satisfies the definition of a candidate IMF. In practice, some stopping criterion should be used to avoid infinite loops. Any suitable stopping criterion can

be used. One commonly used stopping criterion is a Cauchy type of convergence 
test [26]. A check should be made at the end of every step of sifting and it should be stopped when the standard deviation of the result between consecutive sifting, $\sigma$, becomes smaller than a preset tolerance level $T$ (from (5.5) in [26]). If $\sigma<T$ is satisfied, the result of sifting should be declared an IMF. The sifting process is then repeated on the residue. We can also limit the maximum number of iterations allowed for sifting to obtain each IMF, or place a tolerance on the energy of the signal obtained after each iteration, or their combination [78].

\subsubsection{Modified Algorithm}

The new sifting process is given in Algorithm 1.

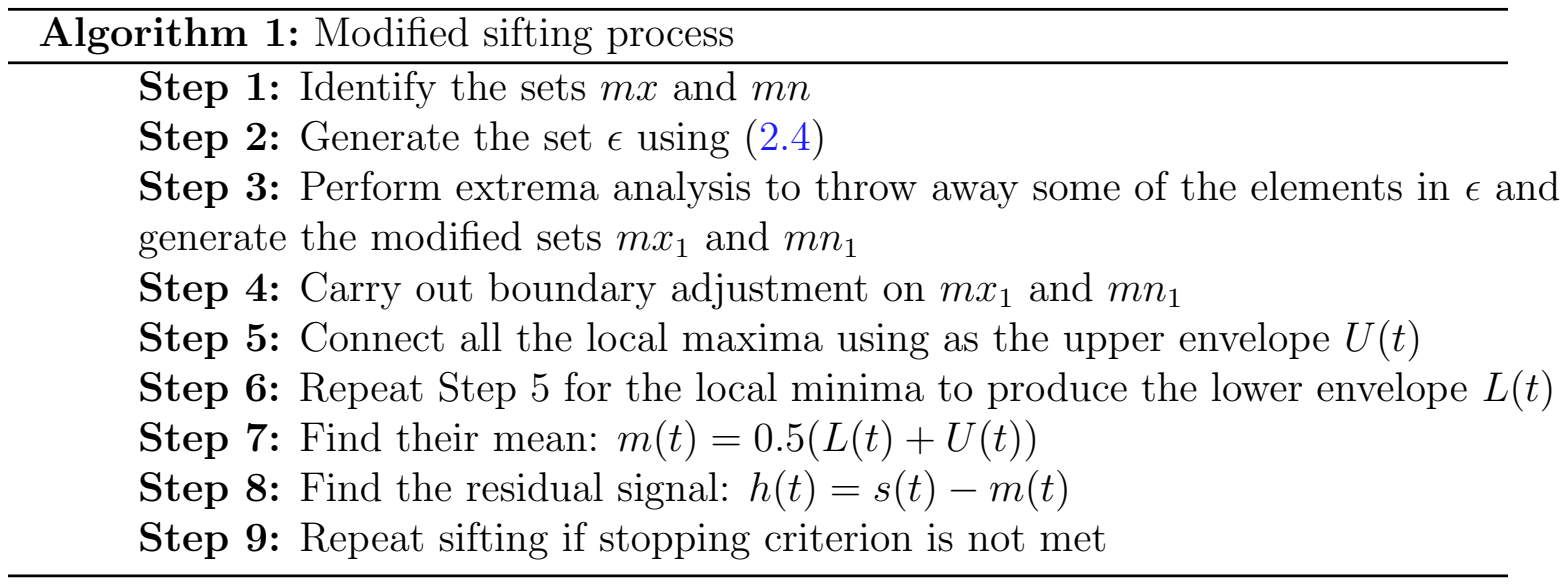

The modified sifting procedure was used to decompose 24 gait cycles, collected using an inertial body sensor node, from a randomly chosen young healthy subject. We found that the gait cycles were statistically similar to each other (Pearson's linear correlation coefficient, $r=0.97$ and p-value, $p<0.0001$ ) and thus, expect to get a similar EMD decomposition for all the cycles. We show that even with an arbitrary small threshold, the modified algorithm is able to decompose most of the cycles into an IMF and a residue, as compared to the original algorithm, which decomposes most of the cycles into two IMFs and a residue. The same threshold is used to decompose 
all the cycles. If, even with the extrema adjustment, a cycle is decomposed into more than one IMF (and possibly a residue), or if the IMFs look different from the IMFs of other cycles, we can look closely at the cycle and find abnormalities, if any. Thus extrema adjustment can be used to indicate the presence of some kind of abnormal behavior.

Fig. 2.4 illustrates the extrema removal process for an example gait cycle. Extrema that are close in value (in amplitude and time) are successively removed, as shown. Endpoints (1st and 33rd samples in this case) were also included in the pruning process.
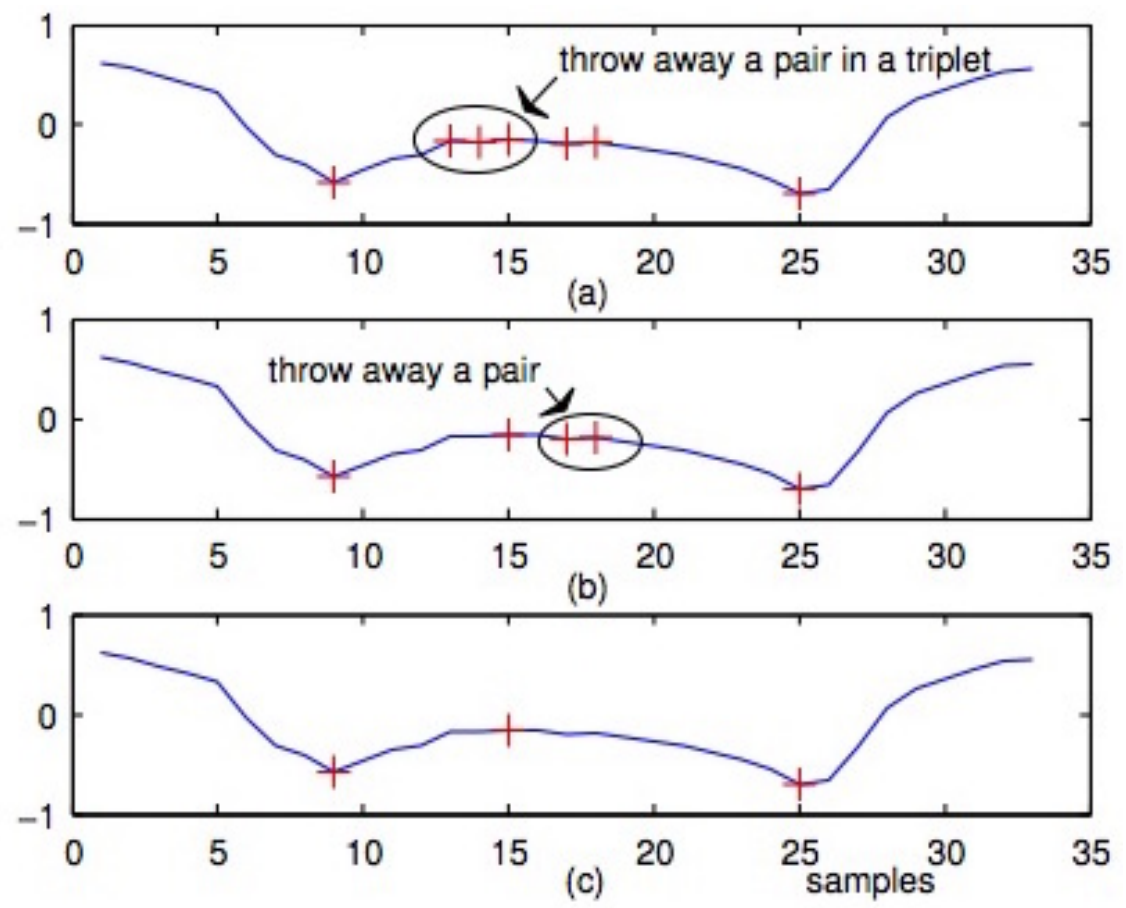

Fig. 2.4: Extrema removal process shown for a gait cycle; (a) original signal with all extrema marked as red pluses, (b) after the first cycle of extrema removal, (c) the final set of extrema marked with red pluses

Fig. 2.5 shows the result of modified EMD on the number and shape of IMFs. The results obtained for two randomly chosen gait cycles are shown. Extrema adjustment helps reduce the number of IMFs. Using the original EMD method, we can throw 
away the first IMF from the second cycle and get a smoother cycle; but we cannot always do this, as cycles could be decomposed into two or fewer IMFs. Instead, by employing extrema adjustments, we get a uniform decomposition on all the cycles without the need to throw away information from signals.

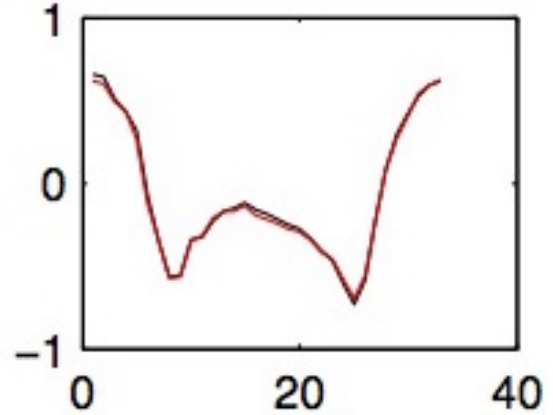

(a)

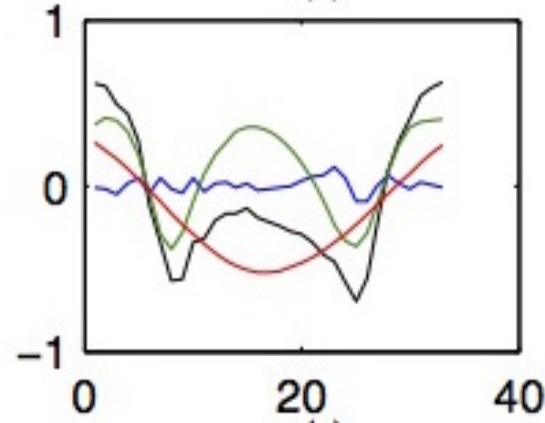

(c)

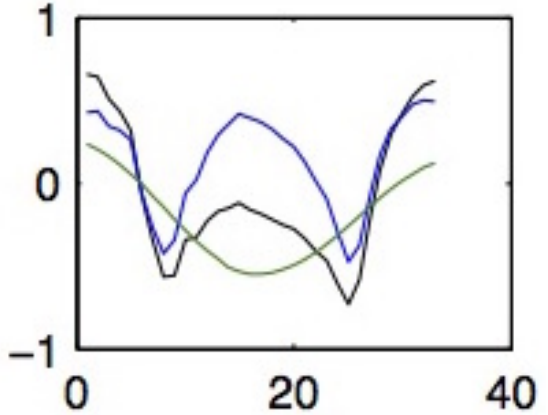

(b)

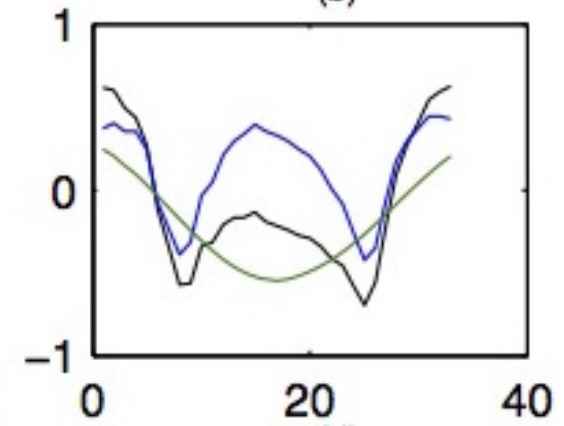

(d)

Fig. 2.5: IMFs of gait cycles; (a) Two gait cycles, first in black and second in red, (b) actual IMFs of first cycle, (c) actual IMFs of second cycle, (d) IMFs of second cycle after extrema adjustment

Fig. 2.6 shows the number of IMFs generated for corresponding gait cycles. Some cycles still have two IMFs and a residue, even after our modification. Upon further investigation, we noticed that using a higher value of the threshold for extrema adjustment makes all the gait cycles decompose into one IMF and a residue. In general, setting a bigger threshold might not be desired, as it will let the algorithm produce IMFs with a larger decomposition error. 

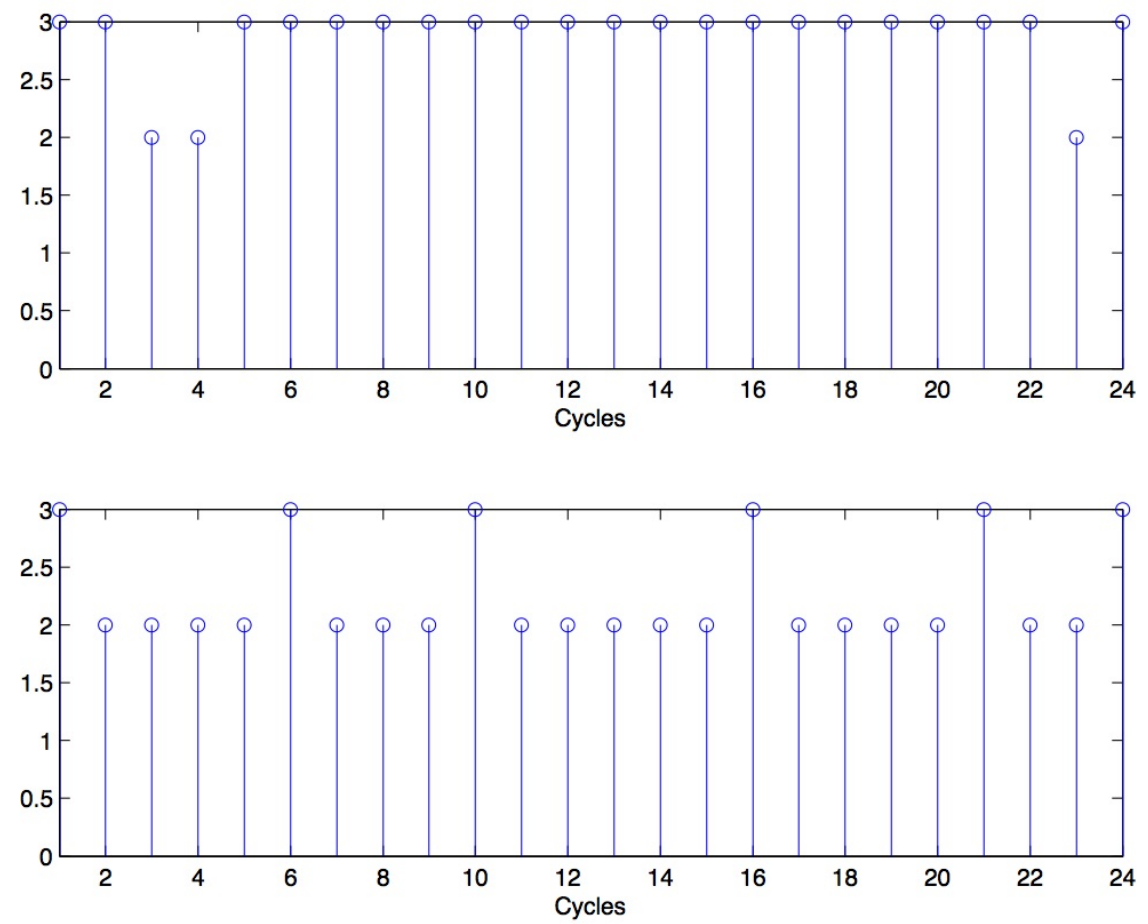

Fig. 2.6: Number of IMFs generated by EMD, last IMF is residue (Top) without any extrema adjustment, (Bottom) with extrema adjustment

The above modification can be employed on data with small sampling errors or with noise in the form of small changes in the signal values. If the signal is itself just one or two IMFs plus possibly a residue, throwing away higher IMFs to remove noise is not a good idea as one might lose important information embedded in those higher IMFs. Instead, we can use our modified version of EMD to get an approximate decomposition and preserve all the signal.

\subsection{The Hilbert Spectrum}

Once the IMFs are obtained, the Hilbert transform is performed on each IMF components [26]. The Hilbert transform of the $i$ th IMF, $\widehat{c}_{i}(t)$, is

$$
\widehat{c_{i}(t)}=\frac{1}{\pi} p \cdot v \cdot \int_{-\infty}^{+\infty} \frac{c_{i}(\tau)}{t-\tau} d \tau
$$


The corresponding analytic signal $x_{i}(t)$ is obtained as

$$
x_{i}(t)=c_{i}(t)+j \widehat{c_{i}(t)}, \text { where } j=\sqrt{-1} .
$$

The polar form of the $i$ th analytic signal in terms of its envelope, $a_{i}(t)$, and its instantaneous phase, $\theta_{i}(t)$, is given by

$$
x_{i}(t)=a_{i}(t) \exp \left(j \theta_{i}(t)\right)
$$

where

$$
a_{i}(t)=\sqrt{c_{i}(t)^{2}+{\widehat{c_{i}(t)}}^{2}}
$$

and

$$
\theta_{i}(t)=\arctan \left(\frac{\widehat{c_{i}(t)}}{c_{i}(t)}\right)
$$

The instantaneous frequency of the $i$ th $\operatorname{IMF}, f_{i}(t)$, is defined as the derivative of the phase

$$
f_{i}(t)=\frac{1}{2 \pi} \frac{d \theta_{i}(t)}{d t} \quad \mathrm{~Hz} .
$$

The envelope and frequency as functions of time can be used to identify structures embedded in the data. The three-dimensional time-frequency-amplitude representation is known as the Hilbert spectrum $H(f, t)[26]$. The Hilbert spectra of the IMFs shown in Fig. 2.1 are given in Fig. 2.7. Note that every successive IMF contains lower frequency content than the previous one. The radius of the circle at a given coordinate $(t, f)$ shows the magnitude contribution from a frequency at a specific time. 


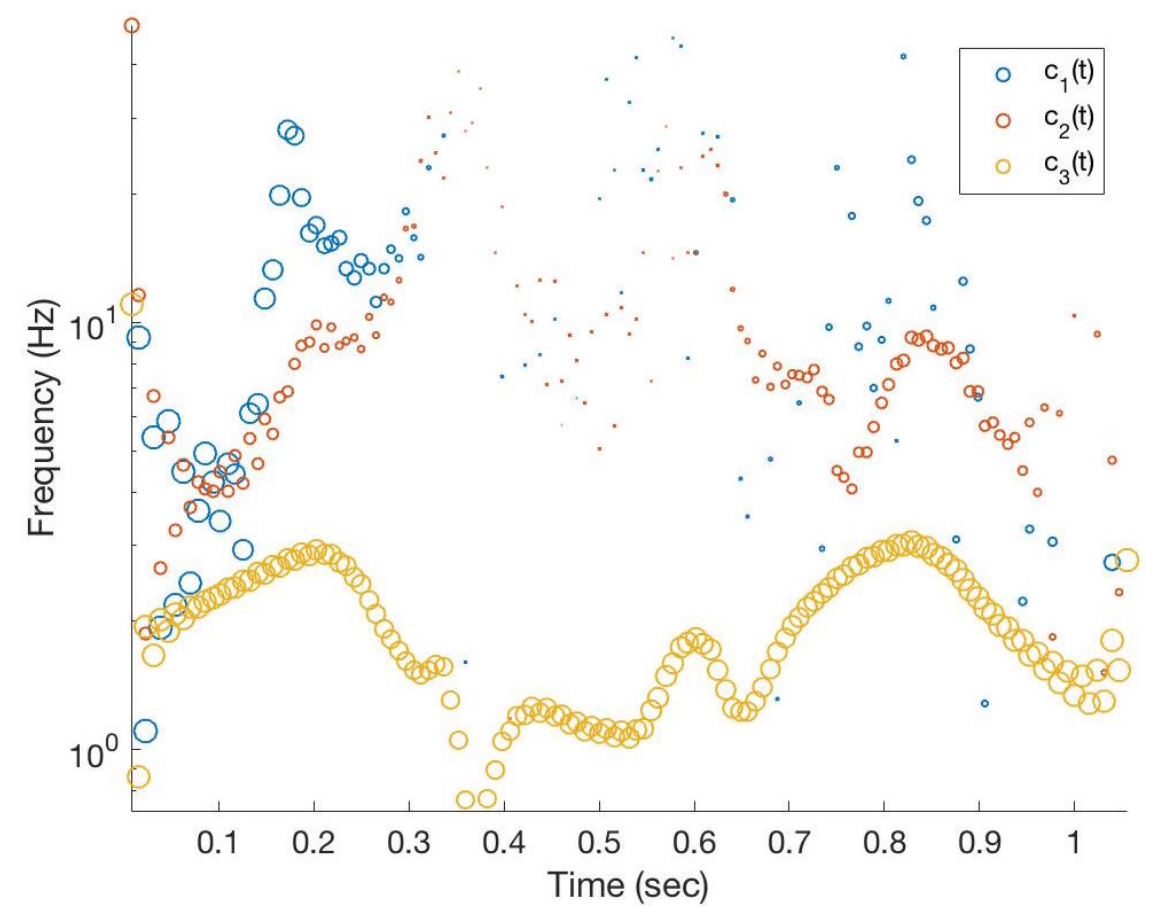

Fig. 2.7: The Hilbert spectra of IMFs of a gait cycle

Ignoring the residue, the synthesis equation in (2.2) can be written implicitly in terms of the Hilbert spectrum as follows

$$
s(t)=\sum_{i=1}^{n} a_{i}(t) \Re\left(\exp \left(j \int_{-\infty}^{t} 2 \pi f_{i}(\tau) d \tau\right)\right) .
$$

Equation (2.11) can be considered a generalized Fourier series with components whose amplitudes and frequencies vary with time to accommodate non-stationary data. The two-dimensional distribution of the amplitude at a given time and frequency makes it easy to visualize which frequencies occurred over the signal time span and where most of the signal energy is concentrated in time and frequency domains, thus allows an understanding of the signal in time and frequency space, simultaneously [26]. 


\subsection{Previous Applications and Modifications of the HHT}

Researchers have used the HHT for data analysis in a number of clinical and research domains including gait studies. The authors of [79] develop an EMD and amplitude/frequency modulation model to classify accelerometry gait patterns. A non-stationary signal analysis based on EMD for gait recognition is proposed in [80]. The potential of EMD for extracting meaningful gait parameters using inertial data from an ear-worn sensor is shown in [38]. Schiecke et al. [81] investigate merits and limitations of EMD and one of its variants to study heart rate variability.

Several research works have modified the original EMD procedure for various purposes. The authors of [82] propose end mirror extensions and employing least square polynomials to lower the errors due to end effects in EMD. The algorithm proposed in [83] uses the above mirror extension technique to lower the errors due to end effects by restricting the splines from varying abruptly at the ends of gaps encountered in real data. The authors of $[76,84]$ propose the concept of a weak IMF by relaxing the requirement that an IMF should be zero mean function. Vatchev and Sharpley prove that any function with simple zeros and extrema can be decomposed into a sum of two or fewer weak IMFs. The decomposition is constructive and it is not obtained by applying EMD [76]. Wang et al. [79] use EMD with modulating techniques to extract features from accelerometer signals and classify walking patterns. Ibrahim et al. [85] classify five different gait patterns using discrete cosine transform features on IMFs of accelerometry data, obtained using a waist-mounted BSN. Yeh [86] proposes a method for computing complex bi-dimensional EMD that could be used to analyze two-dimensional signals. The authors of [87] introduce a convex optimization based 
approach for generating the envelope of a signal during EMD, and show that their method overcomes the shortcomings of spline interpolation. Lee et al. [88] combine nonlinear measures, namely, the weighted mean instantaneous frequencies based on HHT and Lyapunov exponents, to identify a person using his/her gait. The authors of [89] propose a modified version of EMD to separate the amplitude and frequency modulated signals with a better noise performance as compared to others methods.

Filters are also used with EMD. Filters with long impulse responses might mix features far apart in the original signal so may not be desirable to use for signals with transients, while low-pass compact support filters are not smooth enough and thus create artificial oscillations in higher-order IMFs. The authors of [90] propose compactly supported, infinitely differentiable, adaptive local iterative filters to find adaptive and stable decompositions of nonlinear and non-stationary signals. A moving average based approach for a stable EMD analysis is proposed is [91].

\subsection{Summary}

In this chapter, we discussed the commonly used spectral transforms and their limitations in the context of non-stationary data analysis. Since gait is a non-stationary signal, gait analysis using spectral transforms based on a fixed basis and stationary assumption gives only an average state of the gait and does not reflect the changes in the nature of the data occurring during the observation interval, if any. Thus the transform basis should be local and adaptive to the variations in the data in order to accommodate the non-stationary data. The HHT basis, used in this work, satisfies these conditions and is well-suited to gait analysis.

We also presented a simple but effective modification of the original EMD algorithm. Since EMD is sensitive to the location and number of extrema and small 
changes in the data, by including extrema adjustment into the sifting procedure, a modified but more controlled decomposition can be obtained with arbitrary small decomposition error. The HHT procedure can be summarized as shown in Fig. 2.8.

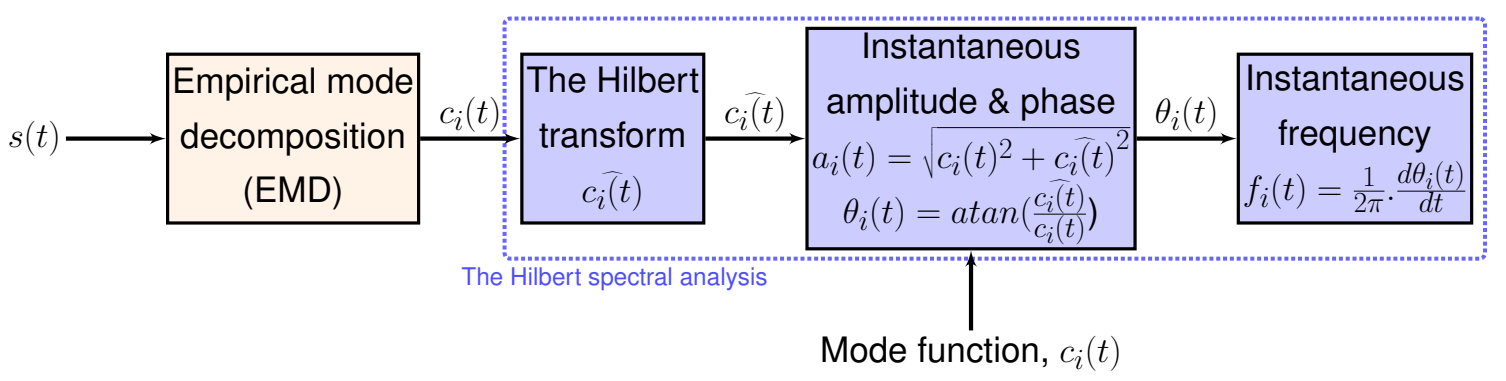

Fig. 2.8: HHT block diagram 


\section{Chapter 3}

\section{CLINICAL MEASURES FOR MS ASSESSMENTS}

This chapter gives an overview of the clinical assessments studied in this work. These assessments include physician-rated metrics, patient-reported outcomes, and the $6 \mathrm{MWT}$.

\subsection{Introduction}

Despite having low variance in ratings, clinical measures, the subject of this chapter, are accessible to physicians. On the other hand, researchers have shown the potential value of using inertial data to extract gait variables as indicators of MS. However, the relationships between gait variables and MS symptoms within and between the MS population specifically, remain under-explored. The relationships between inertial features and clinical measures should be studied for various important reasons. First, such relationships help to know physiological meanings of the gait features making them clinically relevant and easily interpretable by the doctors. Moreover, features might have high variance across study participants but show no clear relationships 
with any subjective assessments or clinical symptoms. Identification of such features is important, since it might be an indication of information that is missing from current subjective assessments, and needs to be captured by new/alternate means. These relationships could also help to personalize current subjective questionnaires, thereby adding value to them. For these reasons, we study the relationships between available clinical data and our inertial features. The standard questionnaires used to record the clinical data are attached in appendix.

\subsection{Types of Clinical Data}

MS is diagnosed by analyzing clinical symptoms, neurological examination findings, and paraclinical metrics (e.g., the results of medical imaging) $[5,7,8]$. In addition, quantitative measures of ambulation, based on timed-walking tests, and subjective assessments are used to monitor the disease progression. Subjective assessments, comprised of questionnaires, can be categorized into two broad categories, namely, physician-rated scores and patient-reported outcomes. A summary of these assessments is given in Table 3.1. Some of the most important metrics are further described below.

\subsubsection{Physician-rated Scores}

Physician-rated scores are based on assessments done by physicians or nurses during the clinical visit. Functional system scale and expanded disability status scale are among the most common examples.

1. Functional systems scores (FSS): FSS are based on a physical exam comprised of seven functional systems, namely, pyramidal, cerebellar, brainstem, sensory, 


\section{Table 3.1: Summary of clinical data categories}

\begin{tabular}{|c|c|}
\hline Category & Description \\
\hline Ashworth scale & Measure of muscles stiffness \\
\hline Bio-data $^{1}$ & Age, sex, height, weight, BMI \\
\hline $\mathrm{BP}^{1}$ & Blood pressure measurements \\
\hline Cognitive & $\begin{array}{l}\text { One of the performance scales to assess cognitive disabil- } \\
\text { ity }\end{array}$ \\
\hline EDSS & $\begin{array}{l}\text { Walking impairment due to MS based on FSS ratings and } \\
\text { gait observations }\end{array}$ \\
\hline Fatigue and depression & $\begin{array}{l}\text { Performance scales measuring MS-associated fatigue and } \\
\text { depression }\end{array}$ \\
\hline FSS & $\begin{array}{l}\text { Seven functional systems: Pyramidal, cerebellar, brain- } \\
\text { stem, sensory, vision, bowel/bladder, and cognitive }\end{array}$ \\
\hline Hand function $^{1}$ & $\begin{array}{l}\text { One of the performance scales, a measure of hands func- } \\
\text { tionality based on a nine hole peg test [92], [93], labeled } \\
\text { as } \mathrm{PS}_{4}\end{array}$ \\
\hline $\begin{array}{l}\text { Instrumental activities of } \\
\text { daily living (IADL) }\end{array}$ & $\begin{array}{l}\text { Eight activities: Using the telephone, getting to places } \\
\text { beyond walking distance, grocery shopping, preparing } \\
\text { meals, doing handyman work, doing laundry, taking med- } \\
\text { ications, managing money, and total IADL score }\end{array}$ \\
\hline MFIS & $\begin{array}{l}\text { Impact of fatigue on cognitive, physical and psycho-social } \\
\text { components }\end{array}$ \\
\hline $\mathrm{MHI}^{1}$ & General psychological distress and mental health \\
\hline $\begin{array}{l}\text { Mobility, sensory, spasticity, } \\
\text { pain, and tremor }\end{array}$ & Five performance scales \\
\hline MSIS $^{1}$ & $\begin{array}{l}\text { Impact of MS on physical and psychological components } \\
\text { of daily life }\end{array}$ \\
\hline MSWS & Impact of motor-related fatigue on walking quality \\
\hline PDDS & Patient rating of disability \\
\hline SF36 & General health status \\
\hline
\end{tabular}

${ }^{1}$ Clinical categories whose sub-scores didn't correlate significantly with inertial features, and thus are not discussed further in this work.

bowel and bladder, visual, and mental functions [7]. Each query is rated by an examiner on a scale ranging from 0 to 5 or 6 .

2. Expanded disability status scale (EDSS): Walking impairment due to MS is classified using EDSS. It is an ordinal scale whose outcomes are based on a neurological examination and gait observations. The scale ranges from 0 (normal neurological examination) to 10 (death due to MS) [7], in steps of 0.5 . 
3. Modified Ashworth: Ashworth scale is used to assess spasticity or muscle stiffness, based on the outcomes of a physical exam [94]. The ordinal scale increases from 0 (normal) to 5 (total stiffness), in steps of 1.0.

\subsubsection{Patient-reported Outcomes}

Patient-reported outcomes (PROs) comprise questionnaires completed by people with MS and later evaluated by physicians. Examples of such assessments are:

1. Multiple sclerosis walking scale (MSWS): MSWS is a 12-item patient-rated measure of impact of motor-related fatigue on walking quality $[48,95]$. The scale ranges from 1 to 5 . Thus the range of total MSWS score is from 12 to 60, with a median value of 36 . The MSWS measures different, although related, aspects of walking than the objective tests such as the 6MWT [11].

2. Modified fatigue impact scale (MFIS): MFIS is a self-reported measure of the impact of fatigue on cognitive, physical and psychosis components [8]. MFIS consists of 21 items rated on a scale from 0 and 4 [10]. Thus the range of the total MFIS score is from 0 to 84 , with a median value of 42 .

3. Mental health inventory (MHI): MHI is a 18-item patient-based measure of general psychological distress [10]. The scores range from 1 to 6 . It is reported to be a reliable tool for mental health assessment [10]. MHI measures the overall emotional functioning and includes a number of negative and positive emotions.

4. Health status questionnaire (SF36): SF36 comprises 11 questions, each containing sub-questions. It is a measure of general health status $[10,96]$. The scale ranges from 1 to 5 . 
5. Multiple sclerosis impact scale (MSIS-29): MSIS is a 29-item patient-reported measure of impact of MS on a the daily life of people with MS [97]. It is comprised of 20 physical and 9 psychological components. The scale ranges from 1 to 5 .

6. Patient determined disease steps (PDDS): PDDS are patient-reported surrogate of EDSS [98], used to evaluate MS disability and progression. The scale is ordinal and ranges from 0 (normal) to 8 (bedridden), in steps of 1.0 .

7. Performance scales (PS): PS are self-reported assessments of health status, and describe 11 different kinds of disabilities persons with MS may have, namely, mobility, hand, vision, fatigue, cognitive, bladder/bowel, spasticity, pain, depression, tremor, and sensory symptoms [92]. The scale is ordinal, and increases from 0 (normal function) to 5 or 6 (total disability), in steps of 1.0. The PS were developed to find easier ways to collect disability related data already captured by other measures such as MSWS and MFIS.

\subsubsection{Physiological Significance of Gait Features}

The physiological significance of our inertial features is identified by quantifying their relationships with four crucial aspects of MS disability, namely, weakness, physical fatigue, balance, and cognitive/concentration, as proposed in [99]. Research has shown that degradation in waling is associated with impaired cognition [100], thus, cognitive/concentration impairment is included. The relevant clinical sub-scores measuring the four aspects of MS disability are grouped together and the proportion of variance in each inertial feature as explained by these categories is studied. Following is the list of the sub-scores comprising each disability:

1. Weakness (lower extremity muscle strength). MSWS (Q3, 4, 8-9), MFIS Q13, and FSS pyramidal 
2. Physical fatigue (sustained physical exertion). MSWS Q6, and MFIS (Q6, $7,10,21)$

3. Balance (balance and stability problems). MSWS (Q4-5, 8-9), MFIS (Q4), and Cerebellar FS

4. Cognitive/concentration (cognitive impairment and fatigue). MSWS (Q12), MFIS (Q1-3, 11-12, 15-16, 18-19), and FSS cerebral

\subsubsection{Quantitative Walking Tests}

Numerous walking tests are used by practitioners to monitor the MS progression. In our work, the data from the six-minute walk test (6MWT) [11] are used. The $6 \mathrm{MWT}$ is used to assess gait impairment by measuring the distance covered by a person in six minutes using a standard protocol [11], and is validated as a reliable measure of walking capacity and motor fatigue [8].

\subsection{Previous Work Correlating Gait and Clinical Metrics}

Many works present in literature have studied the relationships of clinical data to inertial variables to determine their clinical value. Givon characterized MS gait parameters and correlated them with their neurological disability [45]. Martin studied balance and gait impairment in MS patients with mild pyramidal signs, with no pyramidal signs, and controls [46]. In [47], Gong et al. determined the correlations of total MSWS scores and other clinical measures to body sensor measurements. [17] validated warp score as an inertial measure to quantify fatigue-related gait deterioration and showed it had significant correlations to MSWS, MFIS, and two functional 
systems, namely, cerebellar and pyramidal. [49] showed the clinical significance of DTW and warp scores to MS symptoms specific to gait impairment. [50] measured several gait parameters to identify various MS disability groups and control subjects and also found the relationships between those parameters and MSWS scores.

\subsection{Summary}

This chapter is a brief overview of the available clinical data used in the statistical analysis. It is important to study the relationships between inertial features and clinical data. Such relationships help to discover physical meanings of the inertial features and make them clinically relevant and easily interpretable by the doctors. The correlations between inertial measures and clinical data can be used to augment and customize subjective questionnaires based on a patient's personal needs. Features that show strong correlations to patient-reported outcomes are early indicators of MS-related dysfunction. Moreover, features having high variance across study subjects but no clear relationship with clinical symptoms should be identified since they suggest there is a need to capture more clinical information. Thus we study the relationships between clinical data and our inertial features. The clinical data are summarized in Table 3.1. 


\section{Chapter 4}

\section{DATA COLLECTION AND PRE-PROCESSING}

This chapter gives a brief description of the study procedures, the inertial nodes used for data collection, and the pre-processing done on the data before feature extraction. It also elaborates on the gait terminologies and conventions used in this dissertation.

\subsection{Body Sensor Network}

The inertial body sensor network (BSN) nodes known as TEMPO3.1 (technologyenabled medical precision observation) was developed at the University of Virginia [101]. It is a third generation BSN capable of accurately capturing the inertial data at a sampling rate of up to $128 \mathrm{~Hz}$, adequate to capture gait dynamics [102]. The form of the node is similar to that of a wrist watch enabling non-invasive and minimal intervention data collection. TEMPO3.1 has been used in many human subject studies including orthopedic assessment for children with Cerebral Palsy [30], tremor assessment in Parkinson's disease patients [103], gait assessments in patients with normal 
pressure hydrocephalus [23], and others. TEMPO3.1 nodes collect 3 accelerometer axes and 3 gyroscope axes [101], as shown in Fig. 4.1 [15].

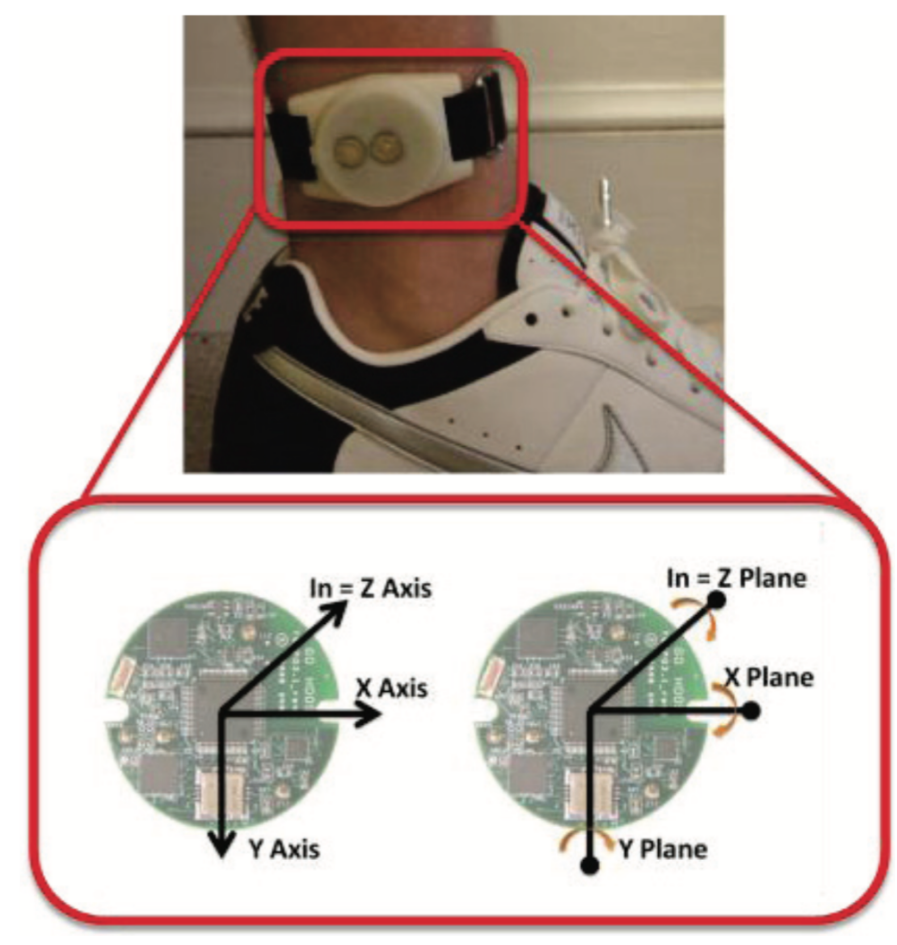

Fig. 4.1: Sensing axis relationships of TEMPO3.1 worn on ankle [35]

\subsection{Study Subjects and Protocol}

The study cohort comprises of 73 MS and 12 comparable control subjects followed for two years, with successive visits being six months apart. Subjects with clinically definite MS [5], categorized into three disability levels, namely, mild (EDSS $0-2.5$ ), moderate (EDSS $3.0-4.0$ ), and severe (EDSS 4.5 - 6.5), based on a neurological examination and gait observations, were recruited from the UVa Neurology department outpatient clinic. The small values of EDSS assigned to mild MS subjects suggest that there are minimal problems in their gait and physical and cognitive functions, and, thus, differentiating them from control subjects using inertial gait markers is of- 
ten a difficult task. All subjects were able to walk for six minutes to safely complete the 6MWT. The exclusion criteria were morbid obesity, known cardiac or respiratory disease, neurological impairment from other diagnoses, or orthopedic limitations. All study procedures were approved by the University of Virginia (UVa) Institutional Review Board. Fatigue-related medications were withheld 48 hours prior to study procedures. Neurostatus-certified staff performed neurological exam for EDSS [7] assessments prior to 6MWT. The MFIS [10] (all subjects) and MSWS [48] (MS only) were collected as well. These details are summarized in Table 4.1.

Table 4.1: Demographics of controls and MS subjects

\begin{tabular}{l|c|c|c|c|c}
\hline \hline & Controls & Mild MS & Moderate MS & Severe MS & All MS subjects \\
\hline \hline Study subjects & 12 & 34 & 28 & 11 & 73 \\
\hline Total visits & 37 & 73 & 57 & 20 & 150 \\
\hline Females & 5 & 18 & 16 & 10 & 44 \\
\hline Age & $37.0(10.8)^{1}$ & $43.0(9.6)$ & $47.0(9.3)$ & $46.0(8.9)$ & $46.0(9.4)$ \\
\hline BMI & $26.5(6.5)$ & $26.6(4.3)$ & $30.3(5.1)$ & MD $^{3}$ & $28.0(4.6)$ \\
\hline EDSS & $1.0(0.6)$ & $2.0(0.5)$ & $3.5(0.4)$ & $6.3(0.6)$ & $3.0(1.5)$ \\
\hline MFIS & $19.0(11.5)$ & $16.0(14.7)$ & $40.0(18.3)$ & $50.0(16.7)$ & $27.0(20.1)$ \\
\hline MSWS & NA $^{2}$ & $12.0(7.4)$ & $26.0(13.6)$ & $56.5(6.9)$ & $19.0(15.9)$ \\
\hline PDDS & $\mathrm{NA}^{2}$ & $0.0(0.8)$ & $1.0(1.4)$ & $5.5(1.4)$ & $1.0(1.9)$ \\
\hline SF36 & $104.0(3.6)$ & $103.0(5.0)$ & $101.0(6.4)$ & MD & $102.0(5.6)$ \\
\hline
\end{tabular}

${ }^{1}$ Median (SD)

${ }^{2}$ Not applicable (NA) indicates clinical sub-scores that were not collected for control subjects.

${ }^{3}$ Missing data (MD)

The 6MWT was completed in a 75-foot corridor using the script given in [8]. To collect the 6MWT inertial gait time series, subjects wore TEMPO3.1 on their wrists, ankles, and the sacrum. Distance was recorded in one minute intervals by hand.

\subsection{Gait Cycle Components}

A gait cycle is comprised of various events that are divided into two main phases, namely, stance and swing $[1,15,104,105]$, as shown in Fig. 4.2. The stance phase 
occurs when the foot is in contact with the ground and makes up $60 \%$ of a normal gait cycle. It is comprised of three sub-phases - heel strike (HS), which starts when the heel touches the ground till just before the toes touch the ground, followed by the mid-stance, which occurs when the entire foot is settled on the ground, and lastly toe-off (TO), when the heel is off the ground and terminates with the toes off the ground as well. The phase between TO and HS is known as the swing phase and makes up $40 \%$ of a normal gait cycle. It is also comprised of three sub-phases - the initial swing, which occurs after TO when forward movement is being made in order to carry the body weight forward (acceleration phase), followed by the mid-swing, when acceleration is maximum, and the terminal swing, which ends when the heel is put on the ground (deceleration phase).

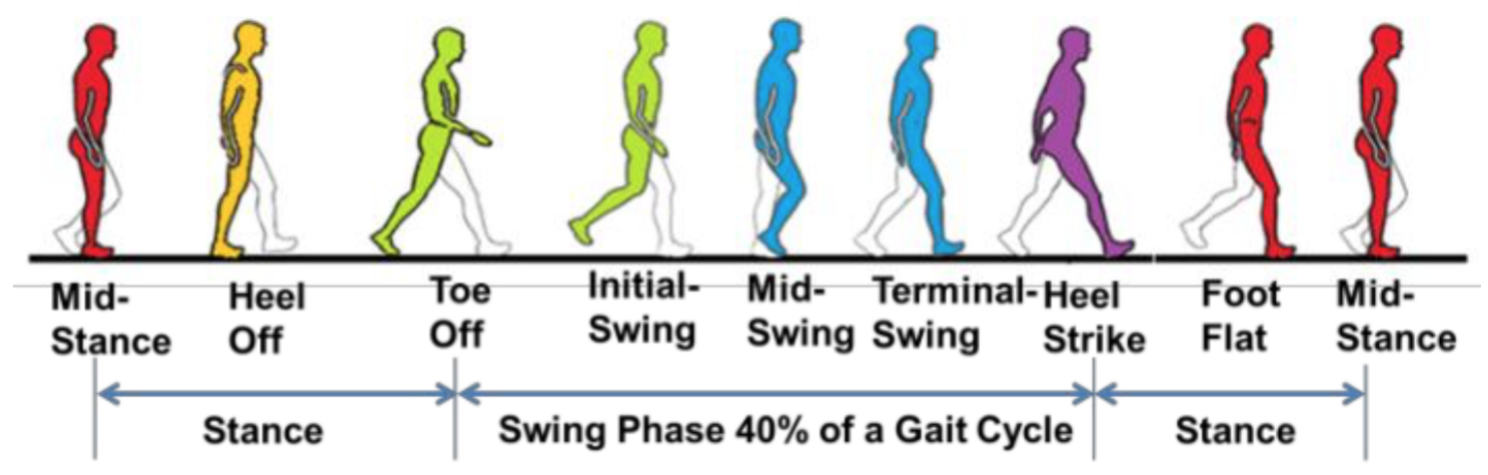

Fig. 4.2: Human gait phases (not drawn to scale) [106]

\subsection{Gait Time Series}

For gait analysis, gyroscope data from the ankles, corresponding to the angular velocity in the $\mathrm{X}, \mathrm{Y}$, and $\mathrm{Z}$ planes shown in Fig. 4.1, are used in this work. The X plane measures "the sideways foot rotation", the Y plane measures "the twisting in a plane perpendicular to the central axis of the tibia", and the Z plane measures "the rotation about the central axis of the ankle" [15]. Angular rate signals are less 
noisy than linear acceleration signals and are used to provide information about the state of the pathological gait, in addition to what is already obtained using the more commonly used linear acceleration signals. We use the magnitude of the three signals to avoid errors due to small deviations in the way the nodes are mounted with respect to the human body frame [32]. The corresponding $6 \mathrm{MWT}$ gait time series, $B(t)$, and gait phases are shown in Fig. 4.3.

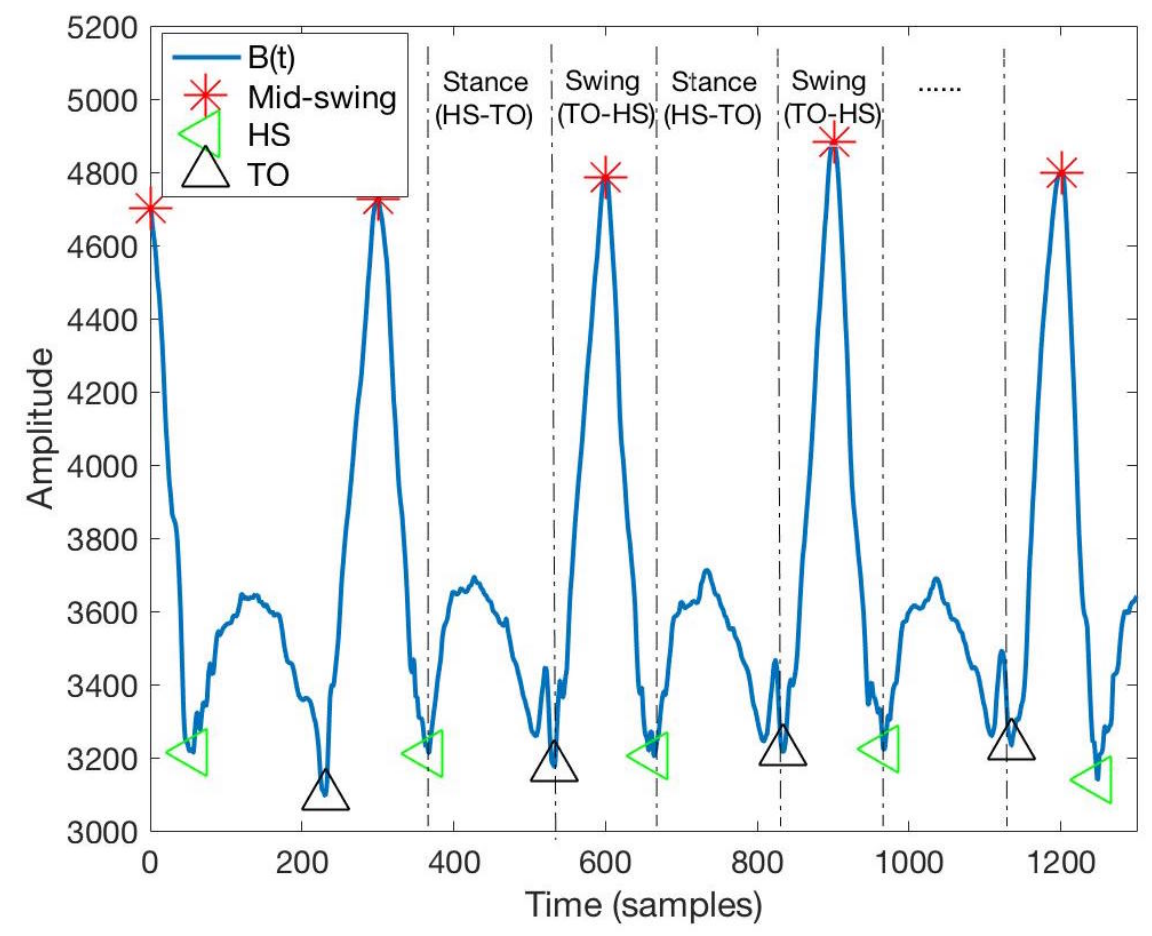

Fig. 4.3: The gait signal $B$ and its components

We use the time-stamps obtained from the inertial node to identify the beginning and end of the signal $B(t)$. At a sampling frequency of 128 samples/s, every 7680 samples represent one minute epoch of the data making the entire 6MWT gait time series $B 46080$ samples long. It should be noted that the prominent peaks present in the signal $B$ correspond to the maximum acceleration during the mid-swing component of gait, making mid-swing easy to identify. To cancel the integration drift [32], the time series between successive mid-swing phases of the same foot is taken as a 
gait cycle or a stride in this work. Stride time, the time to complete one stride, is, thus, the time (samples) between successive mid-swing of the same foot.

For segmenting $B(t)$ into gait cycles, a peak detection algorithm is used to identify the locations of the local peaks corresponding to mid-swing. To avoid detection of multiple peaks surrounding mid-swing, $B(t)$ is filtered using a third-order low pass Butterworth filter. The cut-off frequency of the filter is chosen to be $3 \mathrm{~Hz}$, based on the highest frequency component in the spectrum of $B$. The signal $B$ is then scaled to lie between \pm 1 so that local mid-swing peaks lie over 0.4 amplitude value, as shown in Fig. 4.4, facilitating the identification of gait cycles. Once the peak locations are obtained, the original unfiltered/unscaled inertial data, shown in Fig. 4.3, are used to extract individual gait cycles.

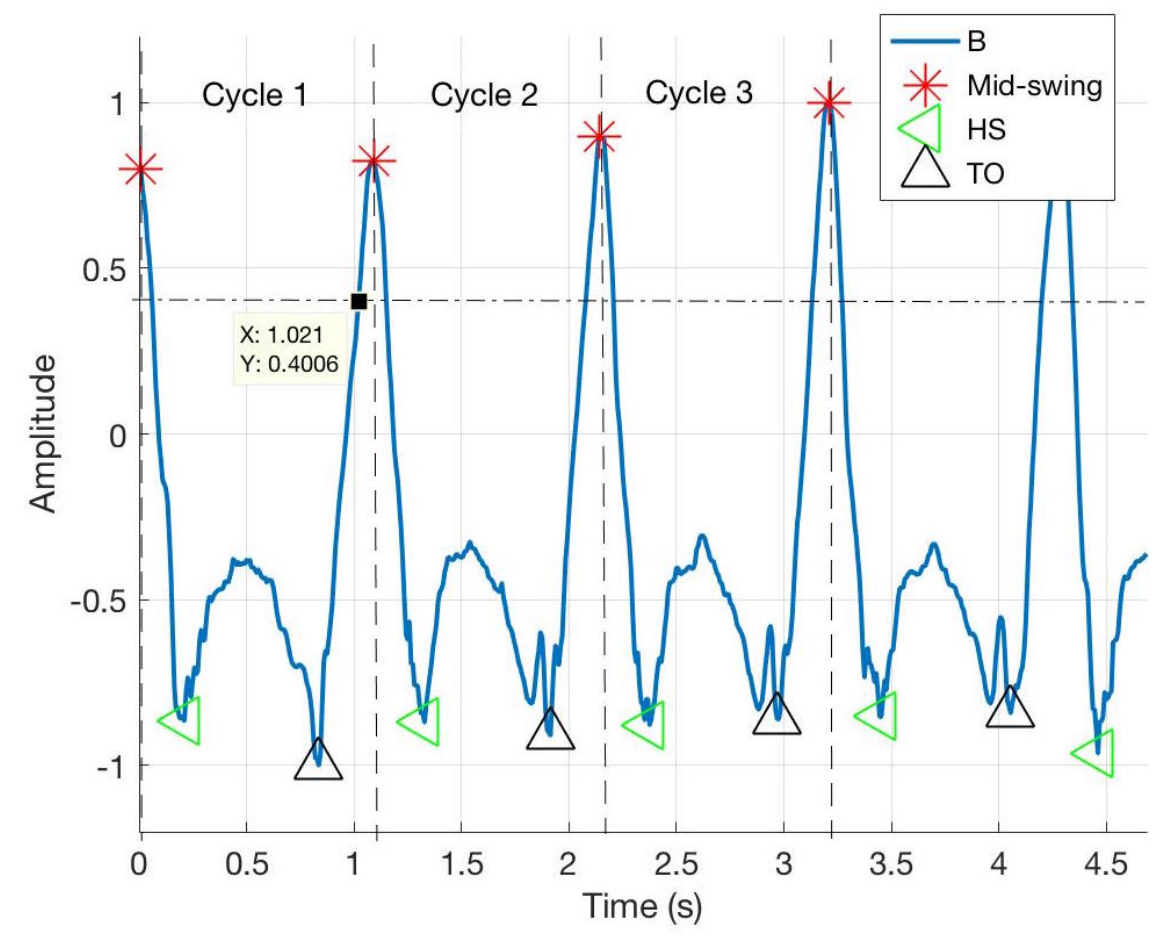

Fig. 4.4: Signal $B$ scaled to \pm 1 . The threshold for detecting peaks corresponding to mid-swing is 0.4 . Gait components and first 3 gait cycles identified. 


\subsection{Summary}

In this chapter, the study procedures, the demographics of the subjects, the description of the inertial sensor platform, and the details of the data pre-processing are described. Fig. 4.4 shows the identified gait cycles and important gait components. 


\section{Chapter 5}

\section{STATISTICAL ANALYSIS}

In order to determine the relationships between feature space obtained from inertial gait data and clinical data of interest, two types of statistical analysis are performed. First, the correlation between inertial features and clinical data is studied to determine which gait features tend to change with the clinical data. Second, linear regression models are employed to give physiological significance of the inertial features. This chapter gives an overview of the statistical analysis methods used in this work.

\subsection{Spearman Correlation Coefficient}

To account for potential non-linear relationships between gait features and clinical data and the presence of ordinal variables, the Spearman correlation coefficient $[107,108]$ is used. In contrast to the Pearson correlation, which assesses linear relationships, the Spearman correlation evaluates monotonic relationship (linear or otherwise) between two variables where variables tend to change together, but not necessarily in a linear fashion or at a constant rate. It is based on the ranked values for each variable rather than the actual data. In the case of Spearman correlation, 
a meaningful relationship can exist between two variables even if the correlation coefficients are close to 0. Pairwise Spearman correlation coefficients $r_{s}$ and $p$-values are reported to demonstrate the relationships of clinical data to the inertial features. The $p$-value is used in statistics to test the hypothesis of no correlation versus the alternate hypothesis that there is a non-zero correlation [109]. A $p$-value smaller than the chosen significance level $\alpha$ indicates that the correlation between two variables is significantly different from 0 . The $5 \%$ significance level, $p<\alpha$, corrected by the total number of comparisons for a given relationship, is used.

Consider two variables $X$ and $Y$, containing $n$ sample points each. If $x_{i}$ and $y_{i}$ represent the $i$ th sample of $X$ and $Y$, respectively, then the Pearson's correlation coefficient $r_{p}$ is a measure of the linear dependence between random variables $X$ and $Y$. Mathematically,

$$
r_{p}=\frac{1}{n-1} \sum_{i=1}^{n}\left(\frac{x_{i}-\mu_{X}}{\sigma_{X}}\right)\left(\frac{y_{i}-\mu_{Y}}{\sigma_{Y}}\right) \approx \frac{\operatorname{cov}(X, Y)}{\sigma_{X} \sigma_{Y}}
$$

where $\mu_{X}$ and $\sigma_{X}$ are the sample mean and sample standard deviation (SD) of $X$, respectively, and $\mu_{Y}$ and $\sigma_{Y}$ are the sample mean and sample $\mathrm{SD}$ of $Y$, respectively. The Spearman correlation coefficient $r_{s}$ is obtained by first converting the raw variables $X$ and $Y$ into ranked variables, $X_{r}$ and $Y_{r}$, respectively, and then computing the correlation between them as follows

$$
r_{s}=\frac{\operatorname{cov}\left(X_{r}, Y_{r}\right)}{\sigma_{X_{r}} \sigma_{Y_{r}}}
$$

where $\sigma_{X_{r}}$ and $\sigma_{Y_{r}}$ are the SD of the ranked variables $X_{r}$ and $Y_{r}$, respectively. Thus the Spearman correlation coefficient is equivalent to the Pearson's correlation coefficient applied to the ranked variables. 


\section{$5.2 \quad$ Linear Regression Model}

Step-wise linear regression models (LRMs) are generated to determine the relationships between a clinical sub-score (dependent variable or response) and the gait features (independent variables or features) $[27,110]$. The step-wise LRM automatically chooses a subset of significant features that explain the response variable in the best possible way. It is a semi-automated process of building a model by successively adding or removing independent variable based on the Akaike information criterion (AIC) [111] performance metric of the model and the t-statistic of the feature with the goal to find the best combination of features to predict the response variable. For every model, the gait features are response variables whereas the clinical scores are the features. Let $X$ be the independent variable and $Y$ be the response variable. Then $\mathcal{M}_{X}^{Y}$ represents a linear regression model, $\mathcal{M}$, predicting the response $Y$ using independent variables $X$, where:

- $X \in$ Clinical data discussed in Chapter 3

- $Y \in$ Inertial gait features and 6MWT speed

Speed is a common clinical anchor used to measure the progression of MS [99], and has been shown to have strong correlation with the overall disability status captured by the EDSS $[99,112]$. Therefore, we have included speed in the regression anal-

ysis to show that our test measures supplement and add value to the speed-based assessments.

The adjusted $R^{2}$, indicating the proportion of the total variation in a dependent variable or response that is predictable from the independent features [113], is reported for each model. A large value of the adjusted $R^{2}$ indicates that a model predicts the response well. To show the $\%$ contributions of the aspects of MS disability 
to the gait features, described in Chapter 3, bar plots are used.

\subsection{Summary}

We use a two-step statistical analysis to determine the relationships between available clinical data and the inertial features. In the first step, a correlational analysis is performed to identify strong and statistically significant relationships between inertial measures and clinical sub-scores. In the second step, linear models are fitted. Spearman correlation coefficients $r_{s}$ and the adjusted $R^{2}$ along with the corrected significance level $\alpha$ are reported for correlational and regression analysis, respectively. 


\section{Chapter 6}

\section{GRADIENT OF THE STRIDE TIME}

This chapter studies the first temporal feature, namely, the gradient of the stride time, which verifies the hypothesis that fluctuations in gait variables over time carry information about the status of the disease. The results show that not only the stride time standard deviation (STSD) is high, but it fluctuates more over six minutes, as the subjects perform the $6 \mathrm{MWT}$, for MS subjects as compared to those of control subjects. The work presented in this chapter was published in [114].

\subsection{Stride Time Standard Deviation}

The STSD is one of the most commonly used markers for MS [112]. Ideally, in normal gait, we expect the standard deviation (SD) of the stride time to be low. If the $6 \mathrm{MWT}$ gait time series $B$ is segmented into $m$ cycles $\left\{C_{i}\right\}_{i=1}^{i=m}$ with every cycle 
being $\left|C_{i}\right|$ samples long, then the STSD, $\sigma_{s t}$, can be calculated using

$$
\sigma_{s t}=\sqrt{\frac{\sum_{i=1}^{m}\left(\left|C_{i}\right|-\bar{B}\right)^{2}}{m-1}}, \quad \text { samples. }
$$

where $\bar{B}$, the average stride time, is given as

$$
\bar{B}=\frac{|B|}{m}, \quad \text { samples } .
$$

The STSD for the given data-set for controls and MS subjects is shown in Fig. 6.1. It can be easily noted that the STSD for MS subjects with lower disability levels $(0 \leq$ EDSS $\leq 4)$ is smaller compared to those with higher disability levels $(4<$ EDSS $\leq 6.5$ ), when computed using the entire $6 \mathrm{MWT}$ gait time series. In fact, $\sigma_{s t}$ for most of mild subjects is found comparable to those of control subjects. Previous studies $[99,112,114]$ have shown that MS subjects slow down while completing the 6MWT, which indicates more variations in the STSD values for MS subjects over time. 


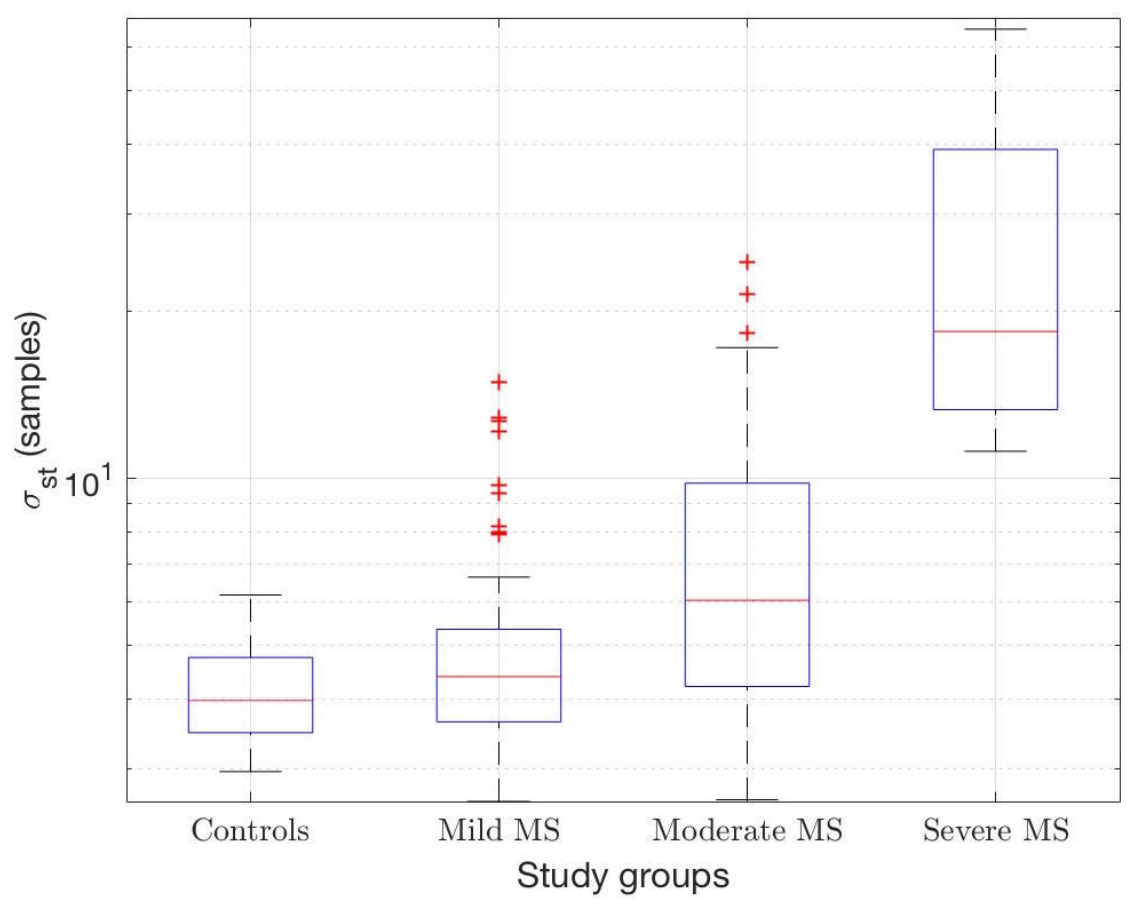

Fig. 6.1: STSD for study groups

\subsection{Gradient of Stride Time Variability over Six Minutes}

Using absolute values for $\sigma_{s t}$ may obscure or average out the changes in stride time, if any, happening over an interval of time. Moreover, occasionally reduced speed, higher/lower STSD, or abnormal ranges for other gait parameters could be encountered due to numerous unknown reasons, including (but not limited to) the fact that the person is a slow walker, his/her mood or general health on the day of the clinical visit, the foot-wear, the walking surface, the motivation behind the walk, etc. Thus, instead of using one absolute value of $\sigma_{s t}$ to assess a subject's walking impairment, we compute $\sigma_{s t_{j}}$ for each minute of the 6MWT, $1 \leq j \leq 6$, and show that the gradient of $\sigma_{s t_{j}}$ over six-minutes are higher, in general, for MS subjects, including those in the low disability category. We can re-write (6.1) and (6.2) for 
minute $j$ as follows:

$$
\sigma_{s t_{j}}=\sqrt{\frac{\sum_{i=1}^{m_{j}}\left(\left|C_{i, j}\right|-\bar{B}_{j}\right)^{2}}{m_{j}-1}}, \quad \text { samples. }
$$

where $m_{j}$ is the number of cycles obtained from minute $j$ and $\bar{B}_{j}$ is the corresponding average stride time, given as

$$
\bar{B}_{j}=\frac{\left|B_{j}\right|}{m_{j}}, \quad \text { samples }
$$

Plots showing $\sigma_{s t_{j}}$ for $1 \leq j \leq 6$ are given in Figs. 6.2 to 6.7. It can be noted that, in general, $\sigma_{s t_{j}}$ fluctuates more for MS subjects than those of control subjects. The fluctuations in STSD are noticeable even for low disability subjects $(0 \leq$ EDSS $\leq 4)$. In fact, towards the end of the walk, the separation between the disability groups increases, with the highest gradient of the STSD found for severe MS subjects. 

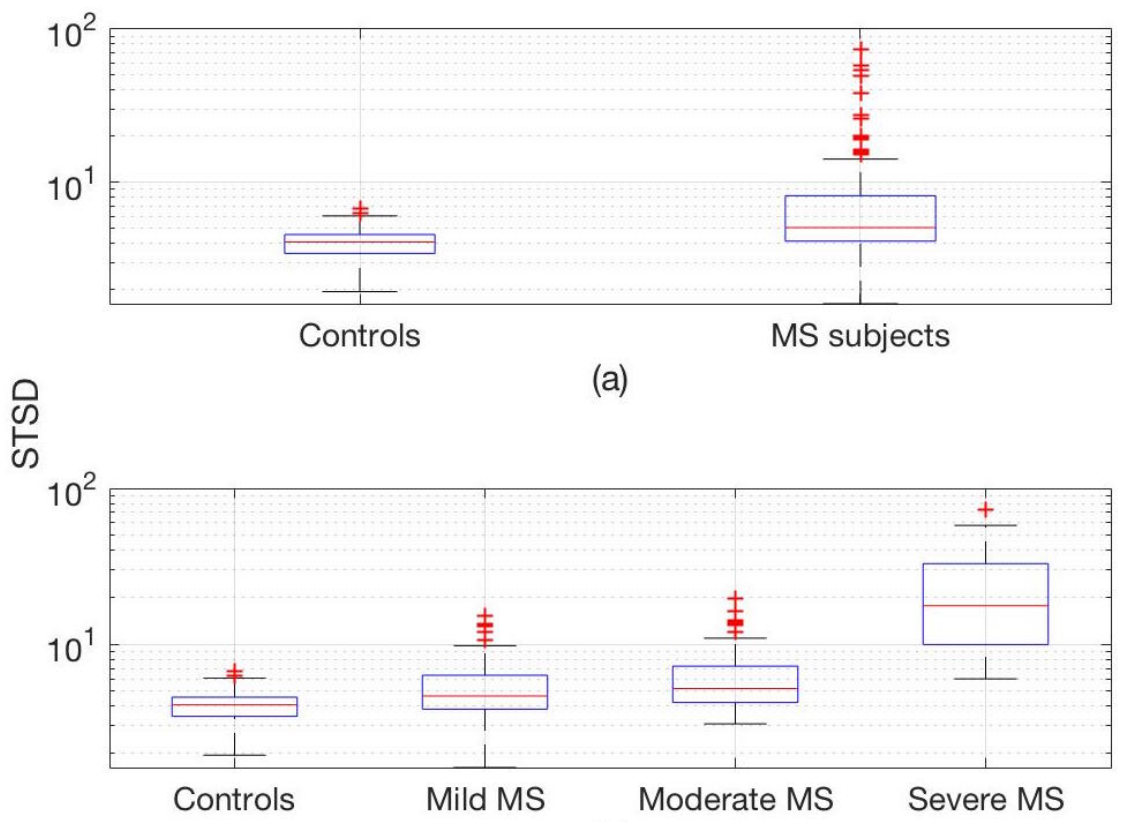

(b)

Fig. 6.2: (a) STSD for minute 1 for MS and control subjects. (b) STSD is shown individually for various MS disability groups

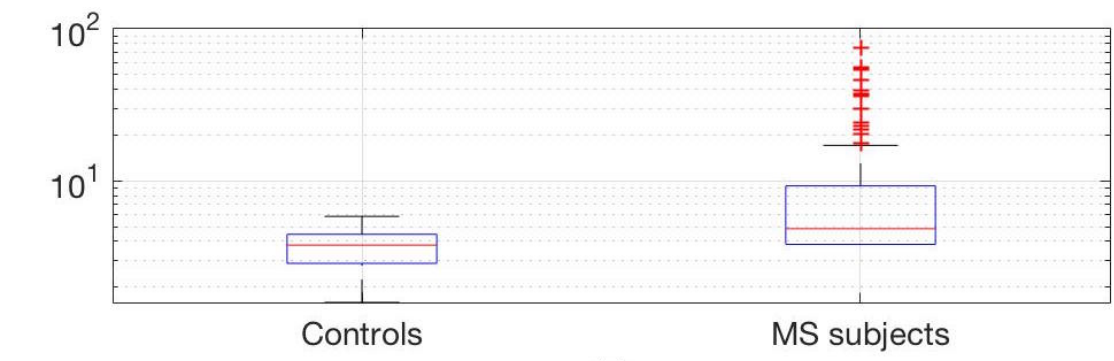

ค

(a)

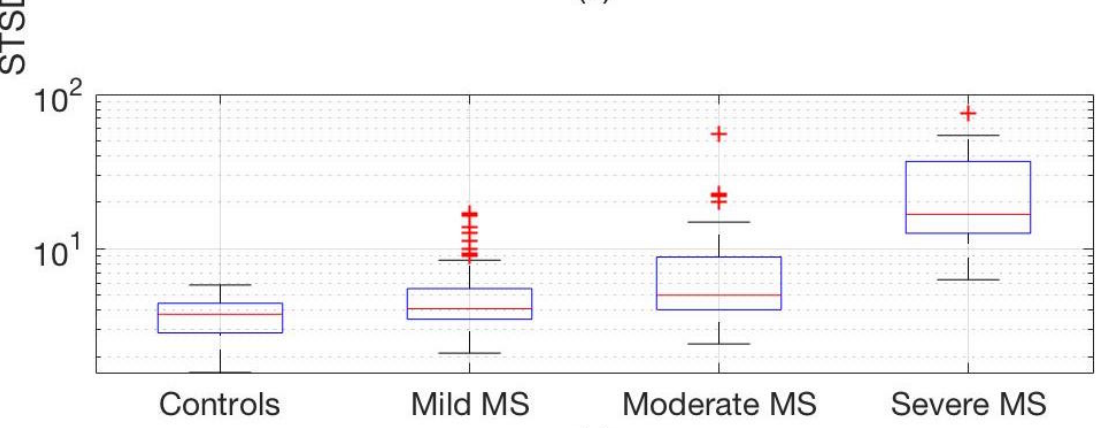

(b)

Fig. 6.3: STSD for minute 2 


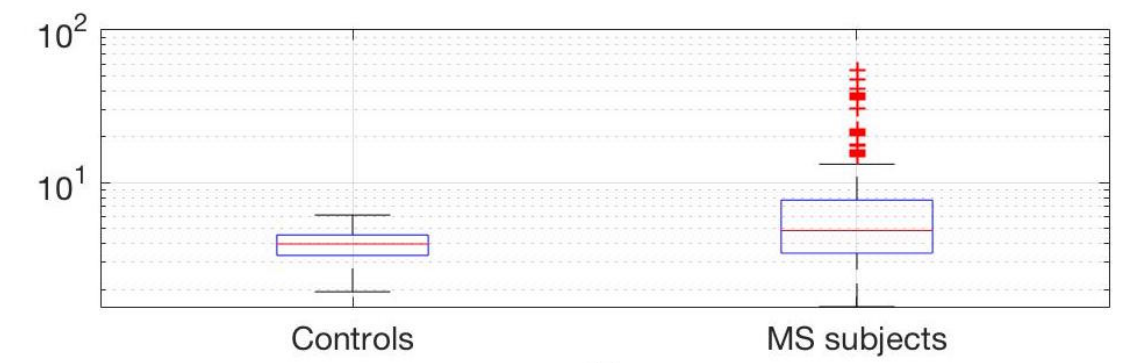

๒

(a)

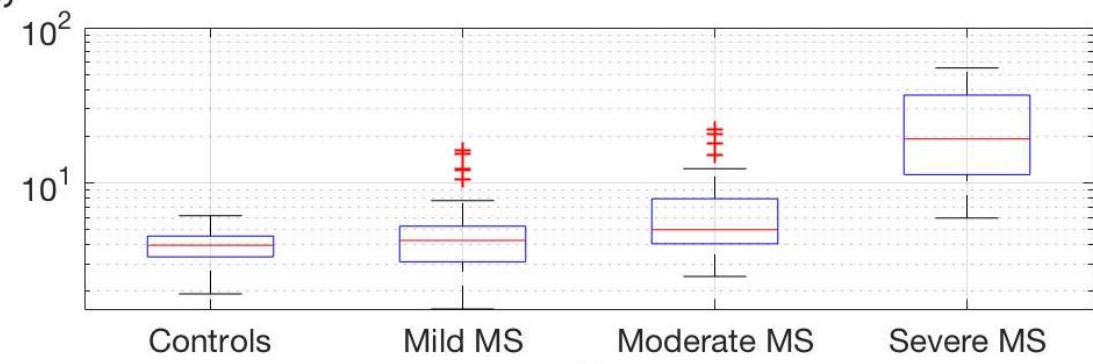

(b)

Fig. 6.4: STSD for minute 3

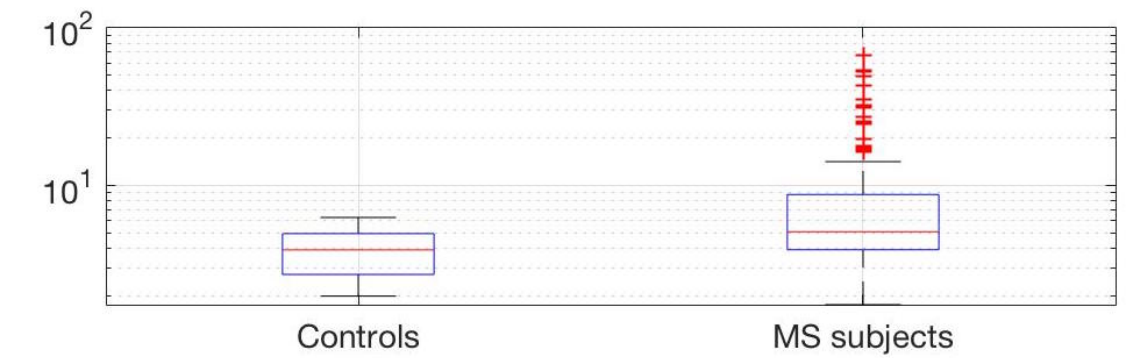

(a)

क

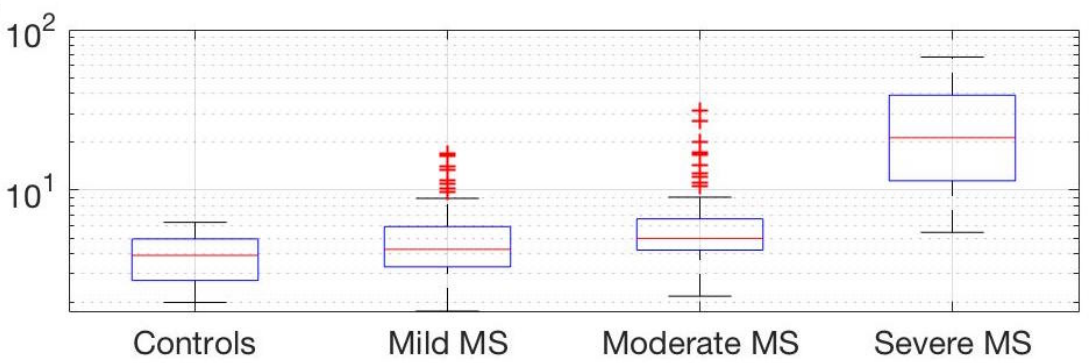

(b)

Fig. 6.5: STSD for minute 4 


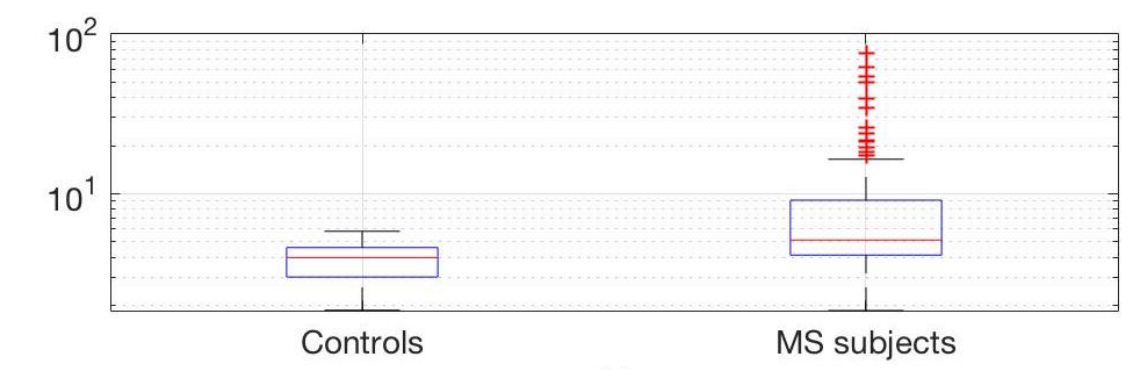

๒

(a)

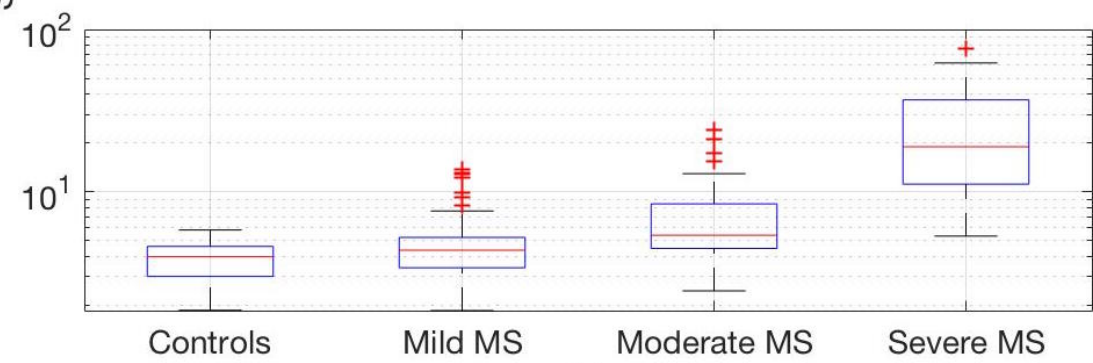

(b)

Fig. 6.6: STSD for minute 5

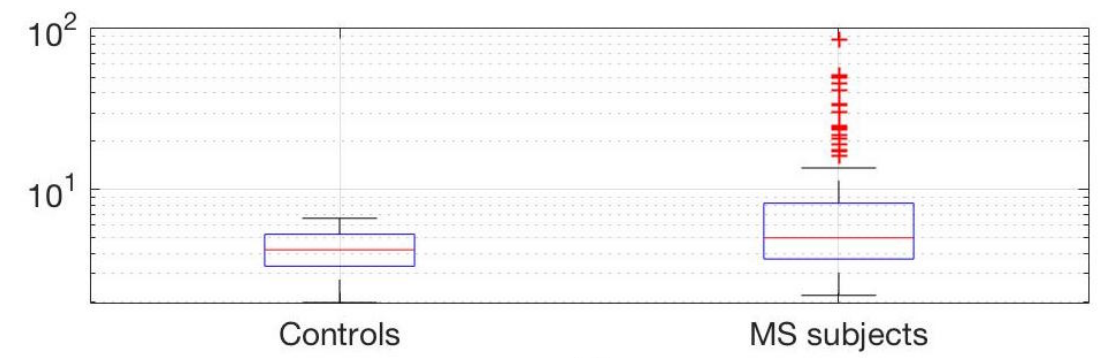

क

(a)

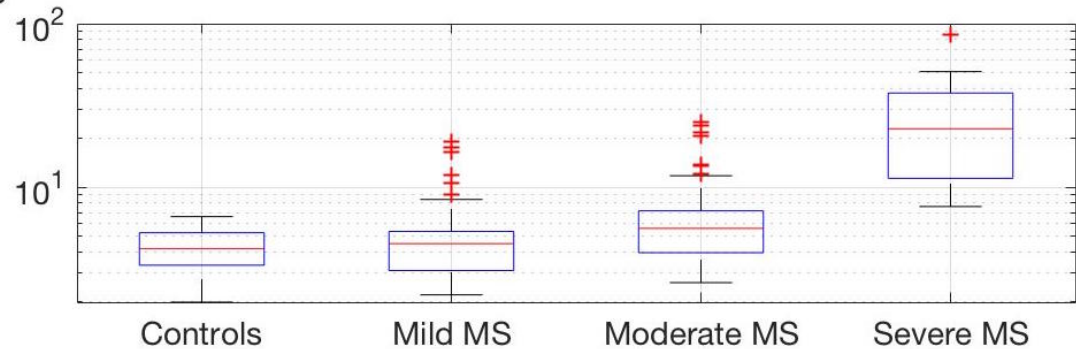

(b)

Fig. 6.7: STSD for minute 6 
The gradient of $\sigma_{s t_{j}}$ is computed as

$$
\Delta_{6 M W T}=\frac{\sum_{j=2}^{6}\left(\left|\sigma_{s t_{j}}-\sigma_{s t_{j-1}}\right|\right)}{6}, \quad \text { samples }
$$

The gradient $\Delta_{6 M W T}$ is plotted in Fig. 6.8, which gives a better insight in the behavior of MS and control subjects' gait. In addition to the gait impairment, gait quality of MS subjects is worsened by MS-induced fatigue. Due to the frequent and strong feelings of fatigue, MS subjects slow down while doing a physical task. Thus the fluctuations in the STSD during the $6 \mathrm{MWT}$ captured by $\Delta_{6 M W T}$ are quite noticeable even for mild MS subjects, making it a better parameter to gauge the impact of MS on a subject's gait compared to the absolute value of the STSD. In the next section, we perform a statistical analysis to determine the physiological significance of stride time gradient.

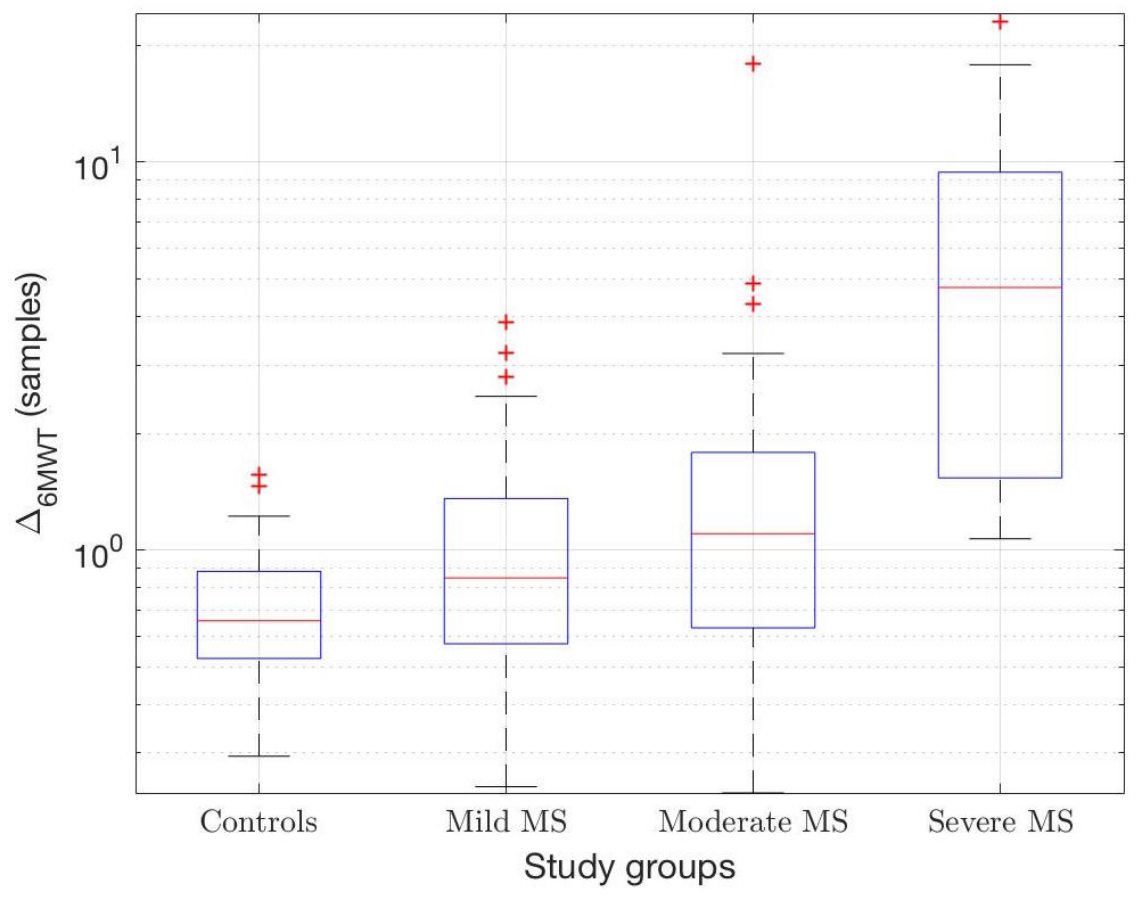

Fig. 6.8: Gradient of STSD 


\subsection{Clinical Significance of Stride Time Gradient}

\subsubsection{Statistical Analysis}

It has been previously demonstrated that MS subjects cover shorter distances and walk at a reduced speed during the 6MWT compared to control subjects [8]. The distances covered by the subjects during the 6MWT, $d$, are plotted in Fig. 6.9. Some MS subjects with EDSS 0 - 4 covered distances similar to those of control subjects. Thus not all MS subjects walked at a slow pace or had a higher gradient of the STSD. To find the clinical reasons behind this, we determine the relationships between $\Delta_{6 M W T}$ and the domains of MS dysfunctions, mentioned in Chapter 3. We show that $\Delta_{6 M W T}$ is smaller (stable STSD for each of the six minutes) for subjects who reported smaller values for disability-related clinical scores.

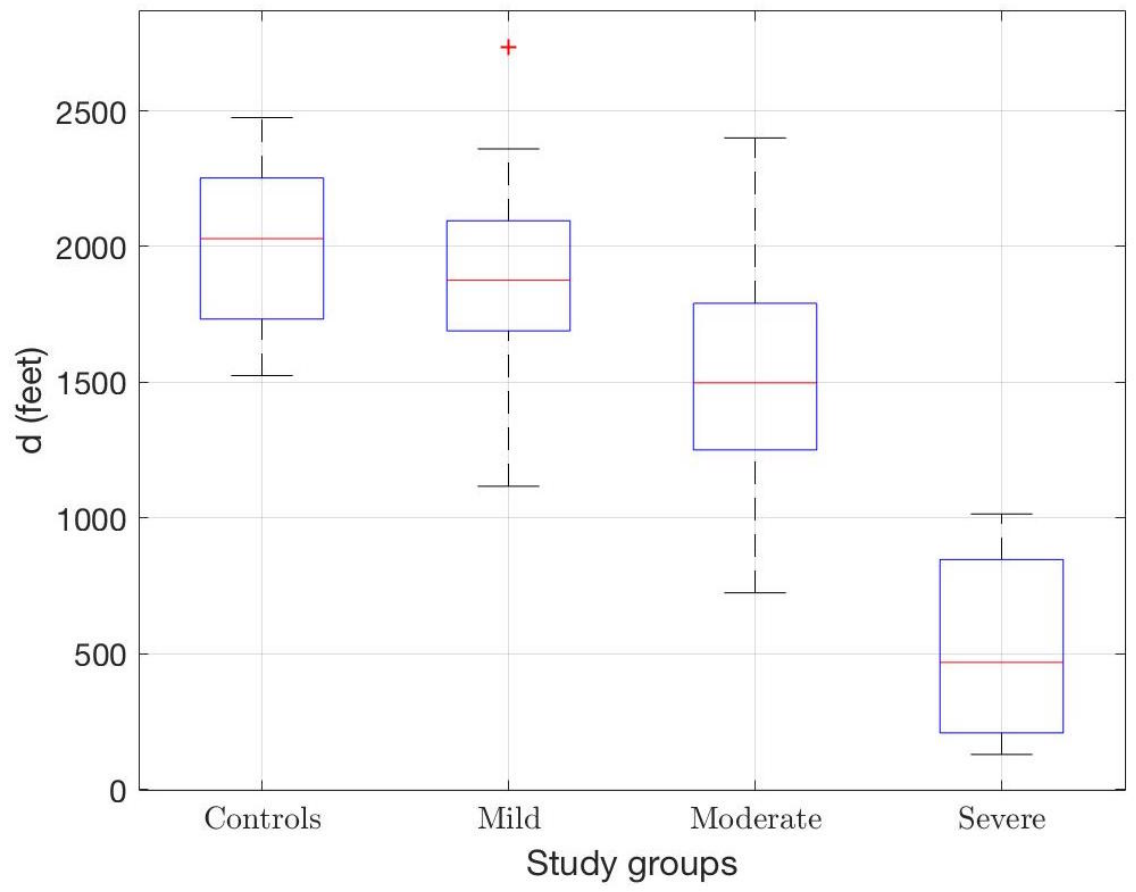

Fig. 6.9: Distance covered during the $6 \mathrm{MWT}$ by study groups

The Spearman correlation coefficients are used to identify clinical sub-scores sig- 
nificantly correlated with $\Delta_{6 M W T}$, shown in Table 6.1. The corrected significance level was found to be 0.0002 . The corresponding LRMs $\mathcal{M}_{X}^{Y}$ are then fitted with

- $X \in\{$ Significantly correlated clinical sub-scores $\}$

- $Y \in\left\{6 \mathrm{MWT}\right.$ speed, $\left.\Delta_{6 M W T}, \sigma_{s t}\right\}$

As mentioned in Chapter 5, 6MWT speed is added as one of the response variables to show the value added by the gradient to traditionally used speed-based assessments. The adjusted $R^{2}$ for the models are reported in Table 6.2. The next section discusses the results of statistical analysis for each clinical categories.

Table 6.1: Spearman correlation coefficient between clinical data and the gradient of STSD, $\Delta_{6 M W T}$

\begin{tabular}{l|c|l|c}
\hline \hline Clinical score & $r_{s}{ }^{1}$ & Clinical score & $r_{s}$ \\
\hline \hline MFIS Q9 & 0.27 & MFIS Q21 & 0.30 \\
\hline Spasticity symptoms & 0.30 & Bladder or bowel & 0.30 \\
\hline Minute 2 distance & -0.31 & Minute 1 distance & -0.32 \\
\hline Minute 3 distance & -0.32 & Minute 4 distance & -0.32 \\
\hline MFIS Q17 & 0.32 & MFIS Q10 & 0.33 \\
\hline Fatigue & 0.33 & Minute 6 distance & -0.33 \\
\hline MFIS Q6 & 0.34 & MSWS Q5 & 0.34 \\
\hline MSWS Q8 & 0.34 & 6MWT speed & -0.34 \\
\hline Minute 5 distance & -0.34 & MSWS Q4 & 0.35 \\
\hline FSS bladder & 0.36 & MFIS Q20 & 0.36 \\
\hline MSWS Q11 & 0.36 & MSWS Q12 & 0.36 \\
\hline MSWS Q2 & 0.37 & FSS pyramidal & 0.38 \\
\hline MSWS total & 0.38 & MSWS Q1 & 0.39 \\
\hline MSWS Q10 & 0.39 & MSWS Q6 & 0.39 \\
\hline FSS cerebellar & 0.40 & MSWS Q7 & 0.41 \\
\hline MSWS Q3 & 0.43 & MSWS Q9 & 0.45 \\
\hline EDSS & 0.45 & PDDS & 0.45 \\
\hline Mobility & 0.46 & \multicolumn{2}{|c}{} \\
\hline \hline
\end{tabular}

${ }^{1}$ The rows are arranged in increasing order of $r_{s}$. 
Table 6.2: The adjusted $R^{2}$ from step-wise LRMs

\begin{tabular}{l|c|c|c}
\hline \hline Clinical category $^{1}$ & 6MWT speed & $\sigma_{s t}$ & $\Delta_{6 M W T}$ \\
\hline \hline Performance scale: fatigue & 26.1 & 13.3 & 8.7 \\
\hline MFIS (MFIS Q6, 9, 10, 17, 20, 21) & 55.3 & 33.3 & 14.4 \\
\hline $\begin{array}{l}\text { FSS (pyramidal, cerebellar, blad- } \\
\text { der) }\end{array}$ & 62.5 & 43.7 & 29.4 \\
\hline Physical fatigue & 63.6 & 29.4 & 16.6 \\
\hline EDSS & 67.1 & 45.8 & 29.9 \\
\hline Cognitive/concentration & 68.9 & 52.5 & 51.5 \\
\hline PDDS & 75.0 & 45.5 & 28.2 \\
\hline $\begin{array}{l}\text { Performance scales: mobility and } \\
\text { spasticity }\end{array}$ & 75.2 & 52.3 & 35.2 \\
\hline MSWS (MSWS Q1-12, total) & 75.4 & 56.9 & 36.6 \\
\hline Balance & 76.7 & 54.4 & 38.9 \\
\hline Weakness & 77.8 & 56.9 & 41.8 \\
\hline \hline
\end{tabular}

${ }^{1}$ From Table 3.1. For each category, clinical sub-scores significantly correlated with the inertial measures are used for regression. The rows are arranged in increasing order of the adjusted $R^{2}$ values for the 6MWT speed.

\subsubsection{Results and Discussions}

In this section, the significantly correlated sub-scores are plotted against $\sigma_{s t}$ and $\Delta_{6 M W T}$. For each plot, the $x$-axis represents clinical scores reported as ordered categories, unless otherwise noted.

1. From statistical analysis, it was found that only three FSS components, namely, pyramidal, cerebellar, and bladder, showed significant relationships to the STSD gradient, as shown in Fig. 6.10 (a-c). Bladder function is a surrogate for assessing spinal cord disease, and thus, both the STSD and its gradient are high for persons with MS whose bladder functions were impaired.

2. EDSS has a strong relationship with the STSD gradient, given in Fig. 6.10 (d). 

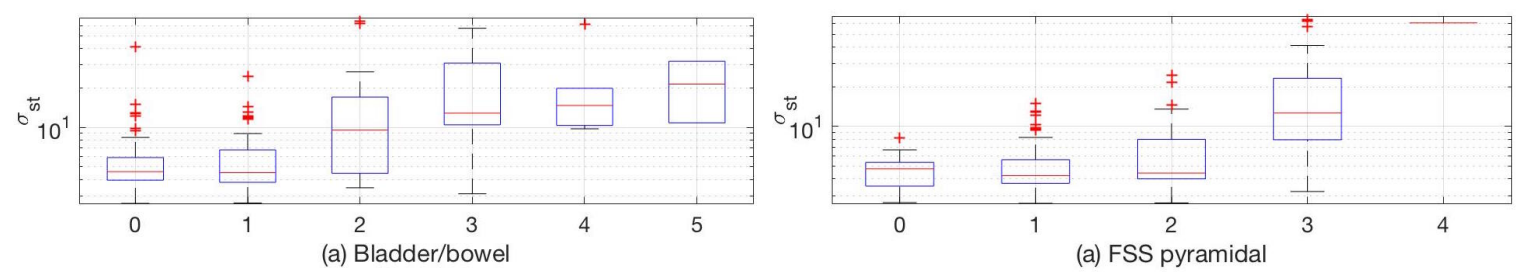

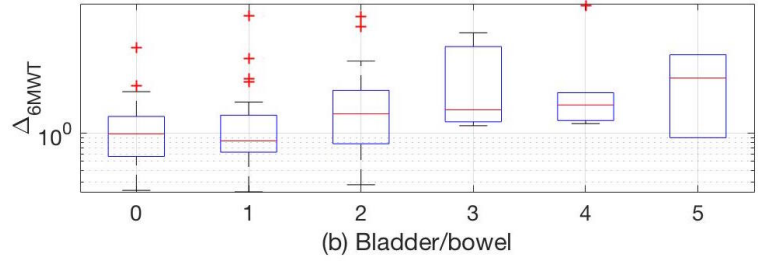

(a) FSS bladder/bowel
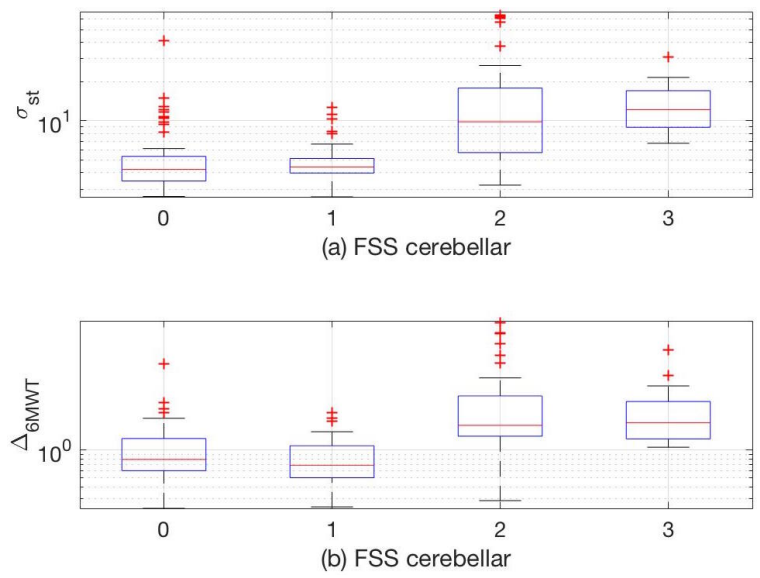

(c) FSS cerebellar

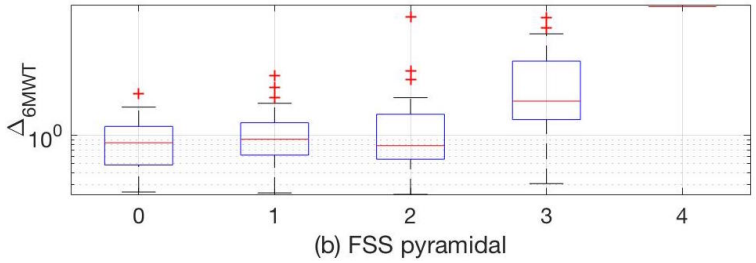

(b) FSS pyramidal
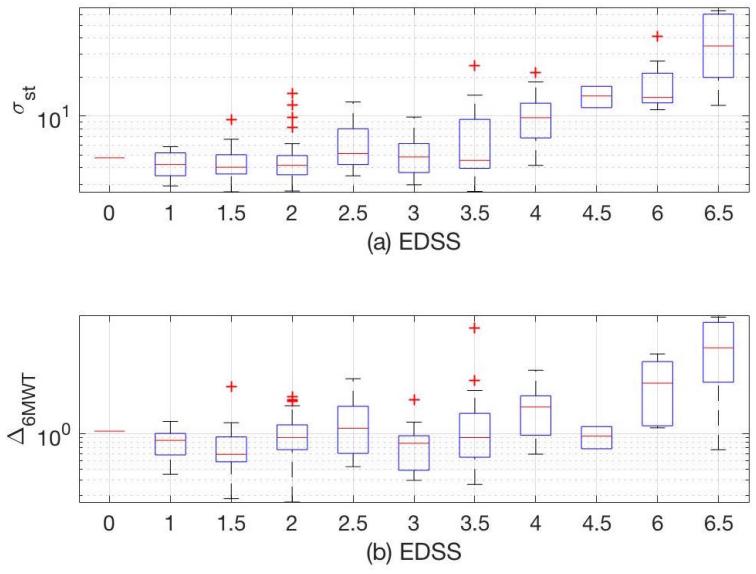

(d) EDSS

Fig. 6.10: Relationships of FSS components and EDSS to $\sigma_{s t}$ and $\Delta_{6 M W T}$

3. MFIS sub-scores as well as total MFIS, shown in Figs. 6.11 and 6.12, respectively, demonstrate weak but significant dependency on the gradient of the STSD. Note that the $x$-axes in Fig. 6.12 represent the STSD and its gradient, respectively, quantized into three intervals for better visualization. 

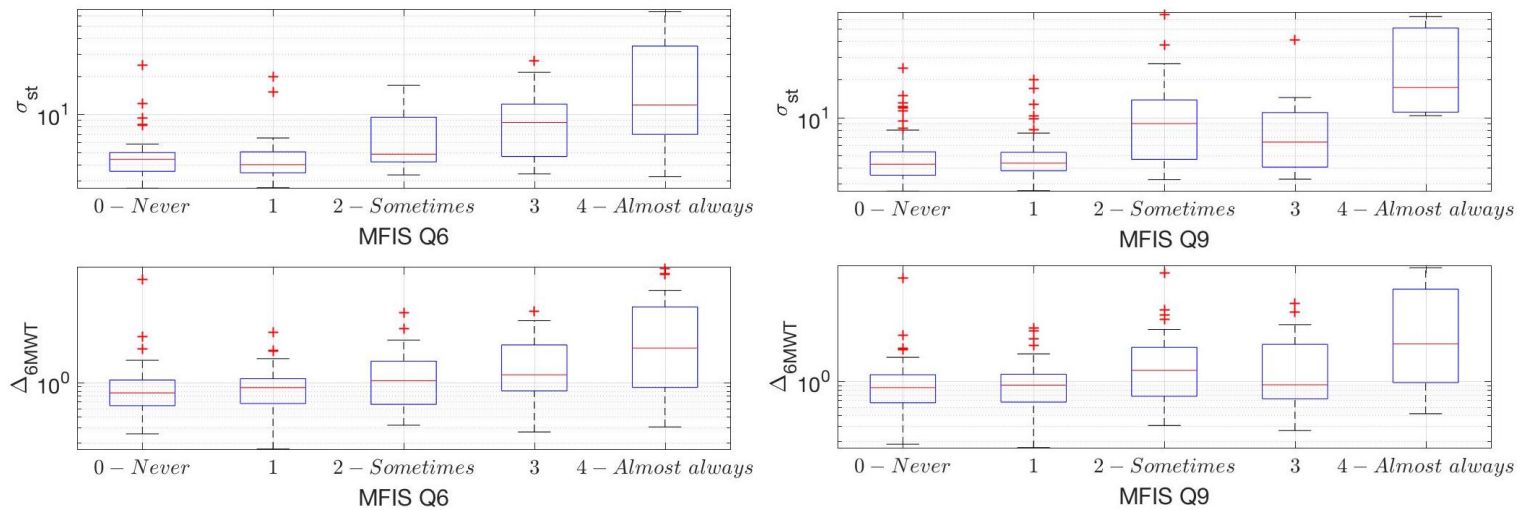

(a) "Pace myself in my physical activities"

(b) "Limited ability doing things away from home"
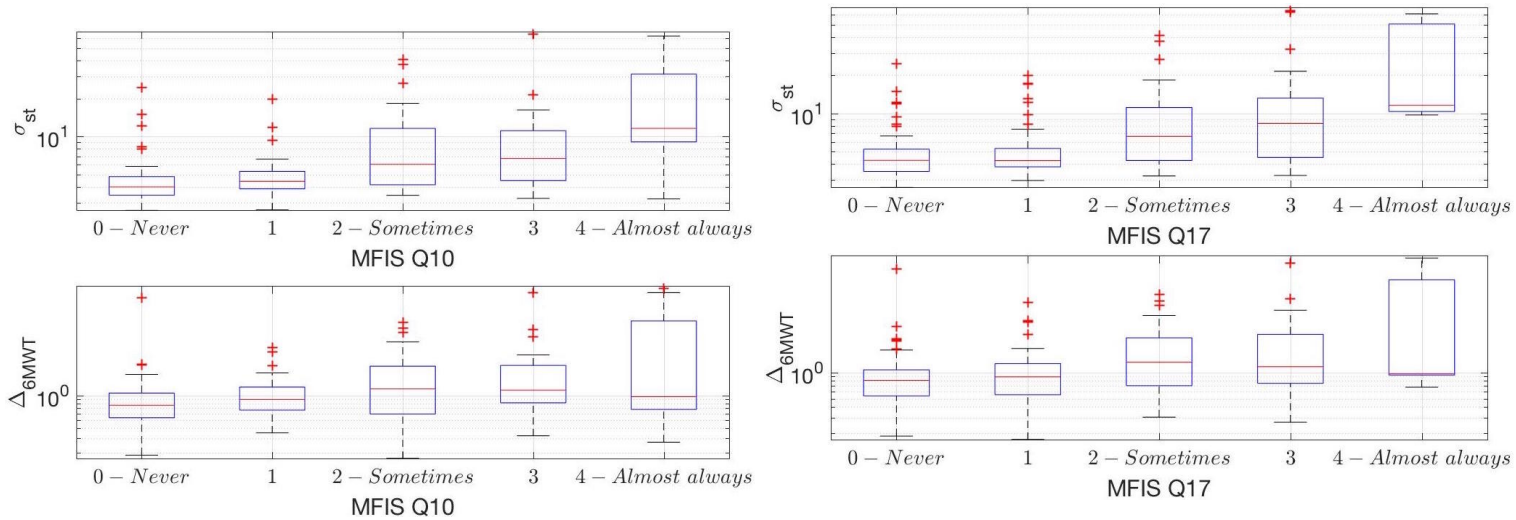

(c) "Trouble maintaining physical effort for long"

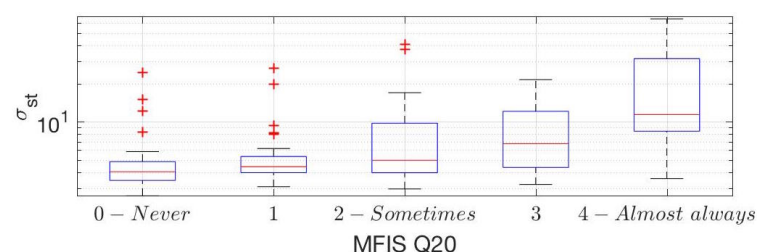

(d) "Less able to complete physical effort tasks"
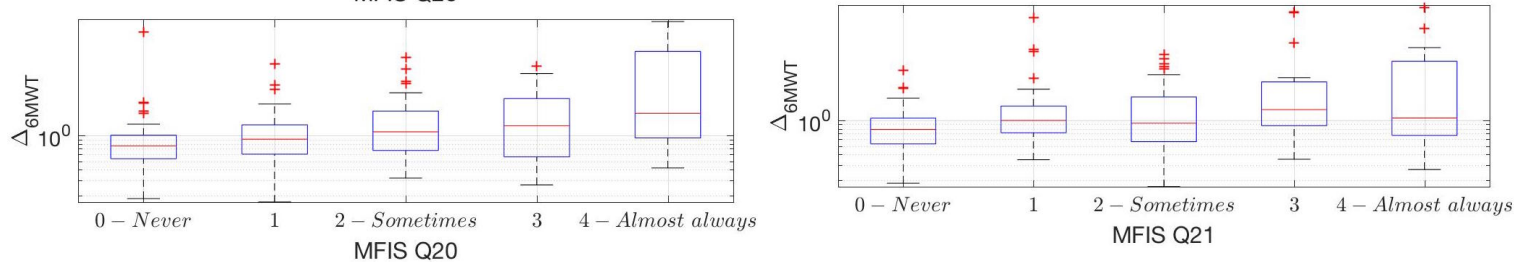

(e) "Limited my physical activities"

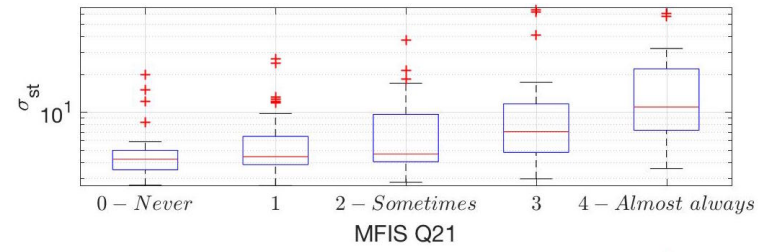

(f) "Needed to rest more often/longer"

Fig. 6.11: Relationships of MFIS components to $\sigma_{s t}$ and $\Delta_{6 M W T}$

4. All MSWS scores had a relationship with the STSD as well as its gradient, although some were weaker than others. Examples for MSWS Q4 and MSWS Q5 are shown 

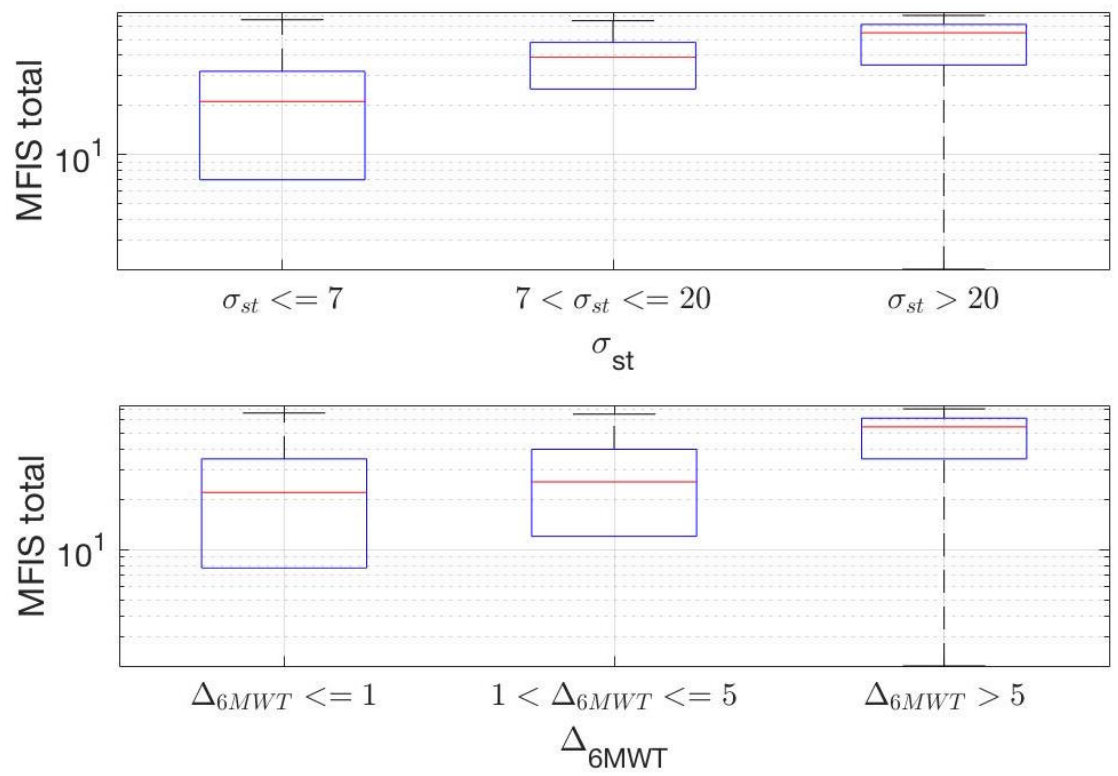

Fig. 6.12: Relationship of total MFIS to $\sigma_{s t}$ and $\Delta_{6 M W T}$

in Fig. 6.13, which showed strong and weak relationship with the stride time variability, respectively.
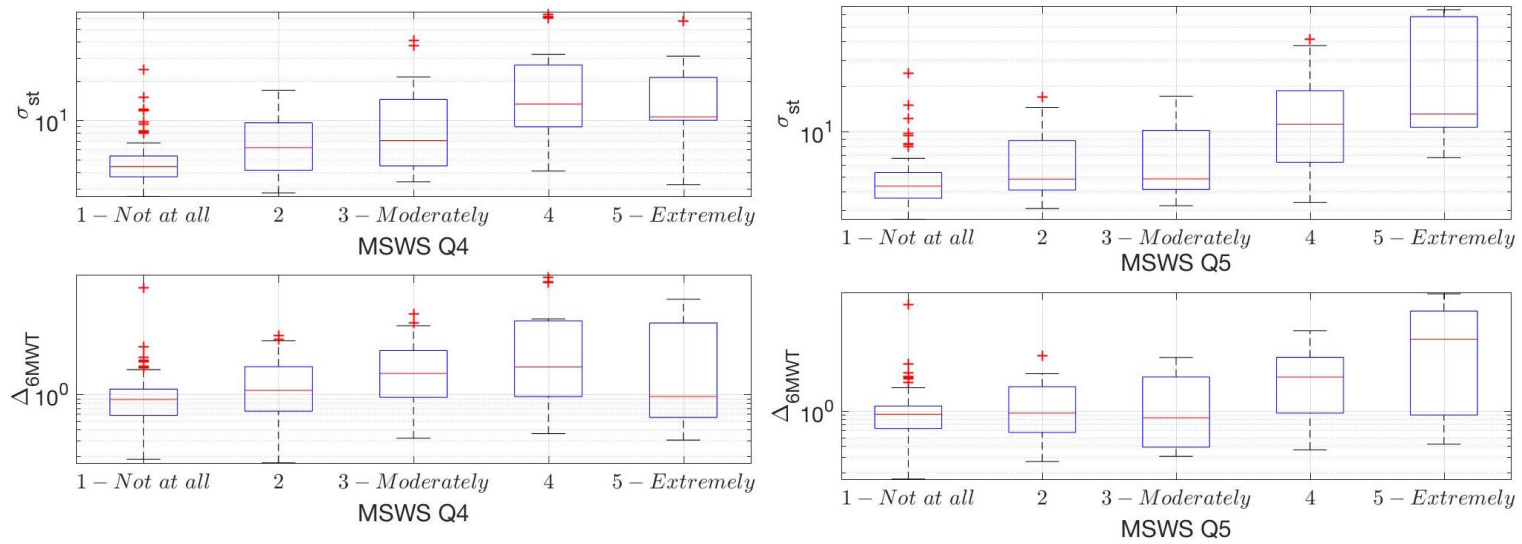

(a) "Standing when doing things
difficult"

(b) "Limited balance when standing or walking"

Fig. 6.13: Relationships of MSWS components to $\sigma_{s t}$ and $\Delta_{6 M W T}$

5. PDDS also showed robust relationships to $\Delta_{6 M W T}$, as shown in Fig. 6.14. 

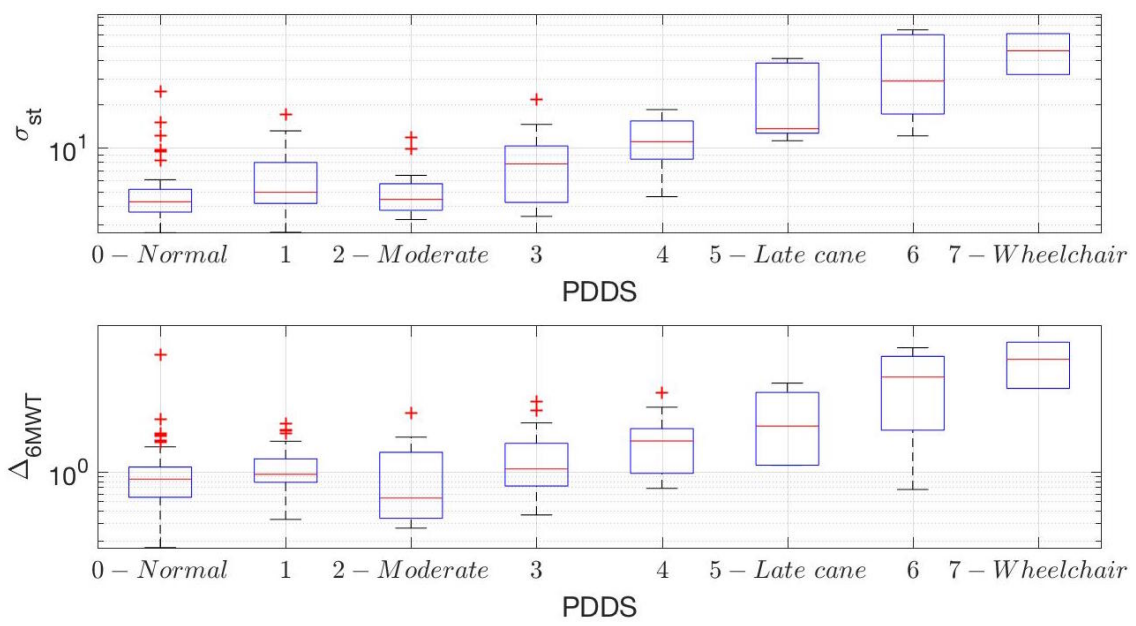

Fig. 6.14: Relationship of PDDS to $\sigma_{s t}$ and $\Delta_{6 M W T}$

6. Fig. 6.15 shows that mobility has a strong relationship with the variations in the STSD.
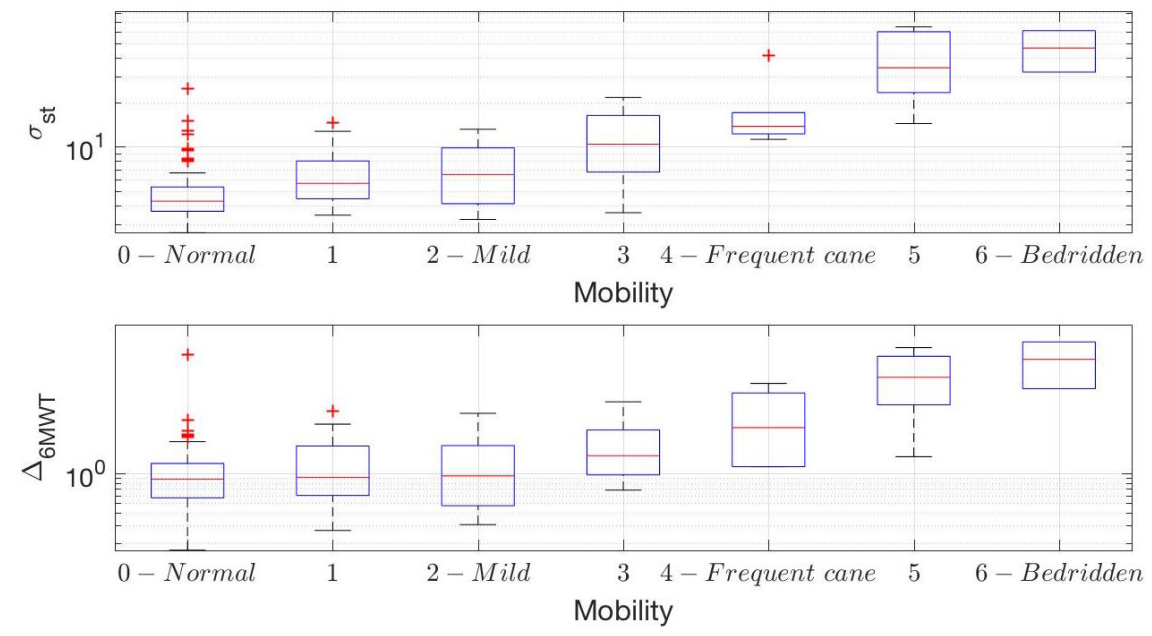

Fig. 6.15: Relationship of mobility to $\sigma_{s t}$ and $\Delta_{6 M W T}$

Relationship of stride time variability to four aspects of MS disability. As can be seen from Table 6.2, the adjusted $R^{2}$ for each of the four MS disability aspects, namely, weakness, physical fatigue, balance, and cognitive/concentration, and $6 \mathrm{MWT}$ speed are over 60 . The strong relationships of the clinical data to speed are intuitive and also confirmed by literature, which show that the 6MWT speed 
has the significant correlation with cognitive and physical impairment in MS, better than the available inertial features, including the gradient of the STSD [17, 99]. The adjusted $R^{2}$ for cognitive/concentration corresponding to both $\sigma_{s t}$ and $\Delta_{6 M W T}$ is a little over 50. The adjusted $R^{2}$ for physical fatigue is smallest $(\approx 16.6)$ for $\Delta_{6 M W T}$. The $\%$ of variance explained by these four disability groups are given in Table 6.3, and their corresponding values are plotted in Fig. 6.16.

Table 6.3: The \% adjusted $R^{2}$ for four aspects of MS disability

\begin{tabular}{l|c|c|c|c}
\hline \hline Gait feature & Weakness & Physical fatigue & Balance & Cognitive/concentration \\
\hline \hline 6MWT speed (fps) & 27.1 & 22.2 & 26.7 & 24.0 \\
\hline$\sigma_{s t}$ (samples) & 29.5 & 15.2 & 28.2 & 27.2 \\
\hline$\Delta_{6 M W T}$ (samples) & 28.1 & 11.2 & 26.1 & 34.6 \\
\hline \hline
\end{tabular}

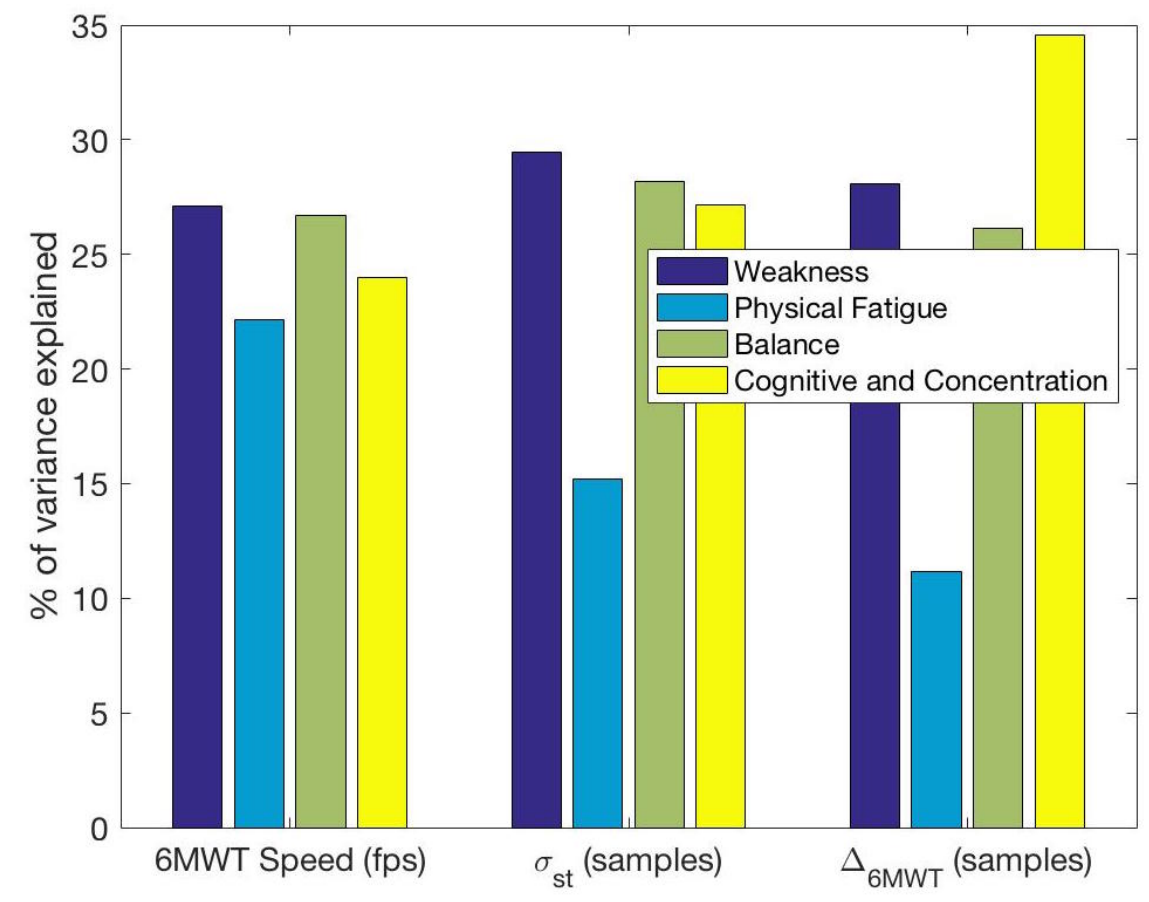

Fig. 6.16: \% of variance explained by the four aspects of MS disability

The 6MWT speed is an overall measure of physical and cognitive deterioration captured by the four categories. The STSD is a uniform indicator of all disability categories but physical fatigue. The gradient is, particularly, the best indicator of 
cognitive/concentration (34.6\%), and least of physical fatigue (11.2\%). This is also evident from individual sub-scores shown above, such as MSWS4. The correlation of the gradient of STSD with the individual disability scores and to the four impairment categories shows that it adds value to speed-based assessments.

\subsection{Summary}

In a normal gait, we expect the strides to be regular, with successive stride times similar. In the controlled setup of the $6 \mathrm{MWT}$, subjects are asked to walk as fast as they can. However, as MS subjects keep walking, they feel tired as a result of fatigue and motor problems, and thus slow down suggesting more variations in their stride time.

This chapter studied the relationships between subjective assessments and change in the STSD over six minutes. The results illustrate that using the gradient, along with the absolute values of STSD, highlights the differences between controls and MS subjects in the low disability categories, even though the overall STSD is low and the distances covered in 6MWT are the same. This is due to the fact that fatigue and impact of MS on physical functions make people with MS slow down while walking over longer duration, yielding variable STSD for different minutes. The effect on gait stride time is higher for subjects for whom fatigue has high impact compared to those who reported fatigue having minimal impact on their functional capacities. The people with MS who reported that fatigue had a minimal impact on them maintained a steady pace while performing the $6 \mathrm{MWT}$, and thus had a low gradient of the STSD.

While absolute assessments are important in order to understand population characteristics, examination of variations in an individual's baseline over time is useful from a clinical point-of-view. Minute-based analysis of the STSD is an example of 
personalized signal processing, by letting physicians observe changes in gait variables over time instead of evaluating the actual/absolute values to remove inter-patient variability. 


\section{Chapter 7}

\section{KERNEL DENSITY ESTIMATION}

This chapter studies the second temporal feature, namely, the kernel density estimate (KDE), which verifies the hypothesis that pathologic gait in MS is restricted in comparison to a healthy gait. The statistics of KDE indeed show that the gait data of MS subjects are less variable than those of control subjects. One feature of KDE, its peak value, is shown to have statistically strong and significant relationships with clinical sub-scores capturing balance and weakness disability in MS. The work presented in this chapter was published in [99, 115, 116].

\subsection{Definition}

Kernel density estimation is a non-parametric statistical method used to estimate the probability density function, known as a kernel density estimate, of a random variable [27, ch. 6]. Kernel density estimation approximates a smooth density function, assuming that it exists, from a finite random sample of the data. The kernel density estimates (KDEs) can be used to discover important features of the underlying 
process.

Let $X$ be the underlying random variable with unknown density function, $f_{X}(x)$. We want to estimate $f_{X}(x)$ using $N$ random observations $\left(x_{1}, x_{2}, \ldots, x_{N}\right)$ of $X$. To approximate $f_{X}(x)$, the kernel method counts the neighbors within a distance $h$ around a point of interest $x$ with weights that diminish with distance from $x . h_{K}>0$ is the so-called smoothing parameter or bandwidth. Specifically, the kernel estimator can be defined as

$$
\hat{f}_{h}(x)=\frac{1}{N} \sum_{i=1}^{N} K_{h}\left(x-x_{i}\right)=\frac{1}{N h_{K}} \sum_{i=1}^{N} K\left(\frac{x-x_{i}}{h_{K}}\right) .
$$

Here $K_{h}(x)=\frac{1}{h_{K}} K\left(x / h_{K}\right)$ is a non-negative kernel function that assigns weights to the neighbors of $x$. Normal kernels are a popular choice, with

$$
K(x)=(2 \pi)^{-\frac{1}{2}} \exp ^{-x^{2} / 2}
$$

\subsubsection{Bandwidth Selection}

A small bandwidth may yield under-smoothed estimates containing artifacts, while a large bandwidth may produce over-smoothed estimates resulting in loss of crucial features of the data. Minimizing the mean integrated squared error, $E\left[\int\left(\hat{f}_{h}(x)-\right.\right.$ $\left.f(x))^{2} d x\right]$, is one of the most commonly used criteria to select an optimal bandwidth. For normal KDE, the optimal value of the bandwidth is

$$
h_{K} \approx 1.06 \hat{\sigma} N^{-1 / 5}
$$


where $\hat{\sigma}$ is the sample SD.

\subsection{Kernel Density Estimates of Gait Data}

To estimate the probability density functions of gait amplitude values, the entire 6MWT gait time series $B$ is used. A normal kernel function with the optimal bandwidth determined using (7.3) is chosen, and the density is estimated at 100 equally spaced amplitude values in the range of $B$. If $b \in B$ represent those 100 amplitude values, then $\hat{f}_{h}(b)$ represents the gait KDE. Fig. 7.1 shows the first 6 seconds of a gait signal $B$ with its corresponding KDE. Note that the density is largely uni-modal, concentrated between amplitudes 3200 and 3600 .
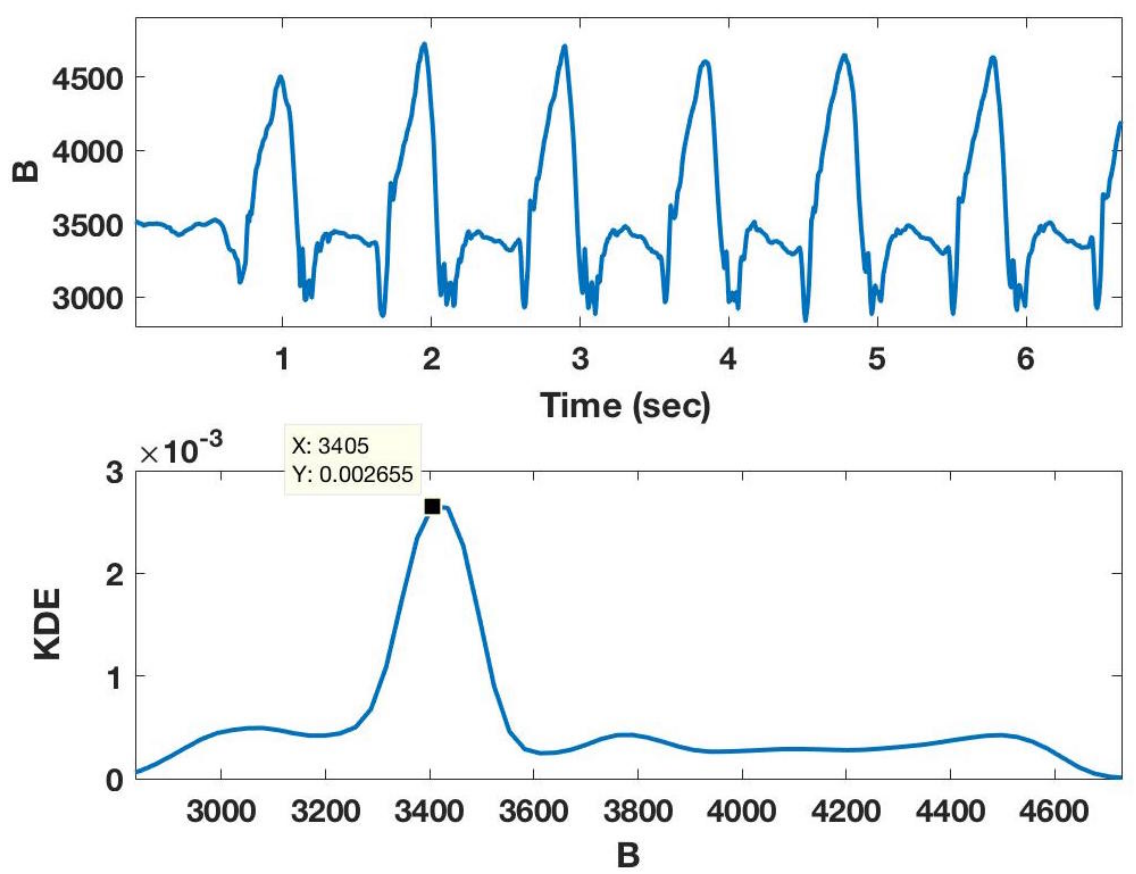

Fig. 7.1: (Top) First 6 seconds of the 6MWT gait data, $B(t)$, for an MS subject, (Bottom) the KDE for the entire 6MWT gait time series. The KDE peak and the location at which peak occurs is shown

The typical densities for MS and control subjects are shown in Fig. 7.2. The KDE can have two peaks - a higher left and a lower right. The KDEs for control subjects 
are found to be bi-modal whereas the MS subjects' gait KDEs are mostly uni-modal. In addition, the KDE peaks for MS subjects are significantly higher, compared to those of control subjects, as evident from Fig. 7.3. This means that, for MS subjects, the difference in gait amplitude among different gait phases is small.

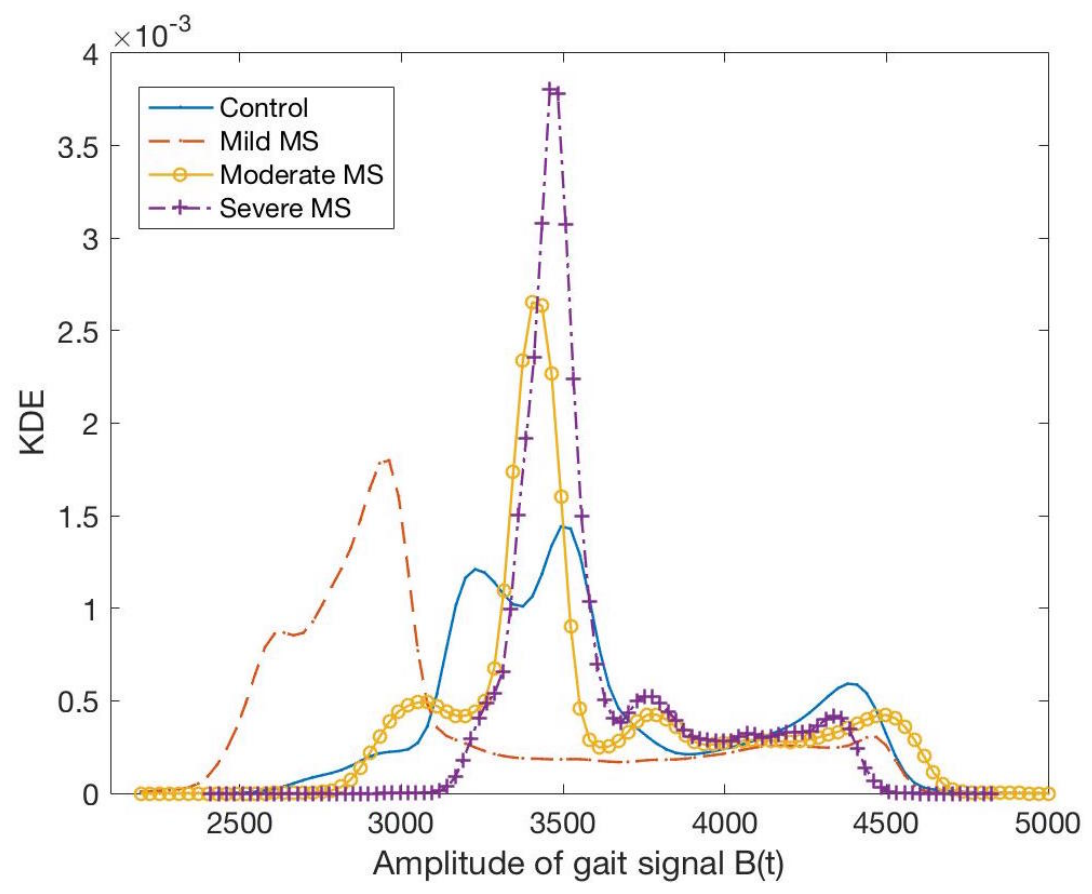

Fig. 7.2: Typical KDEs of gait time series for various study groups 


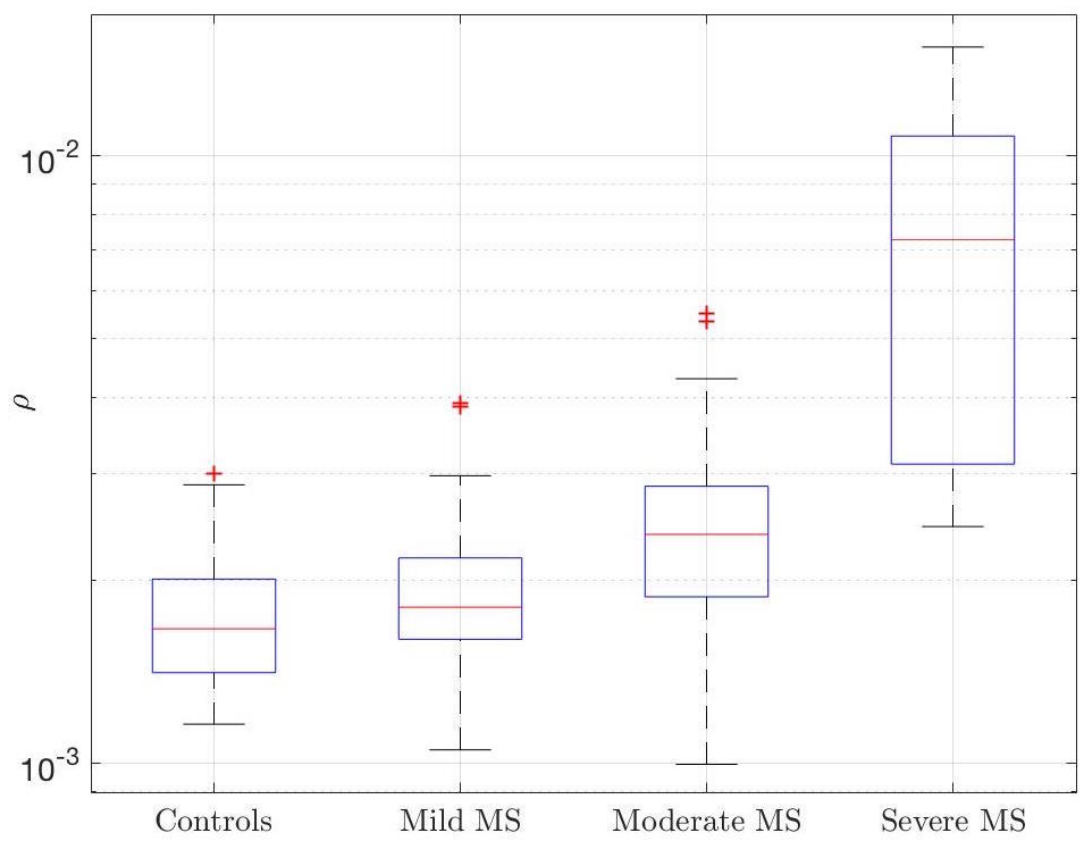

Fig. 7.3: KDE peaks for various study groups

The gait differences are not always present, and thus, some MS and control subjects were found to have similar densities. Fig. 7.4 shows typical and atypical gait densities of MS and control subjects. 


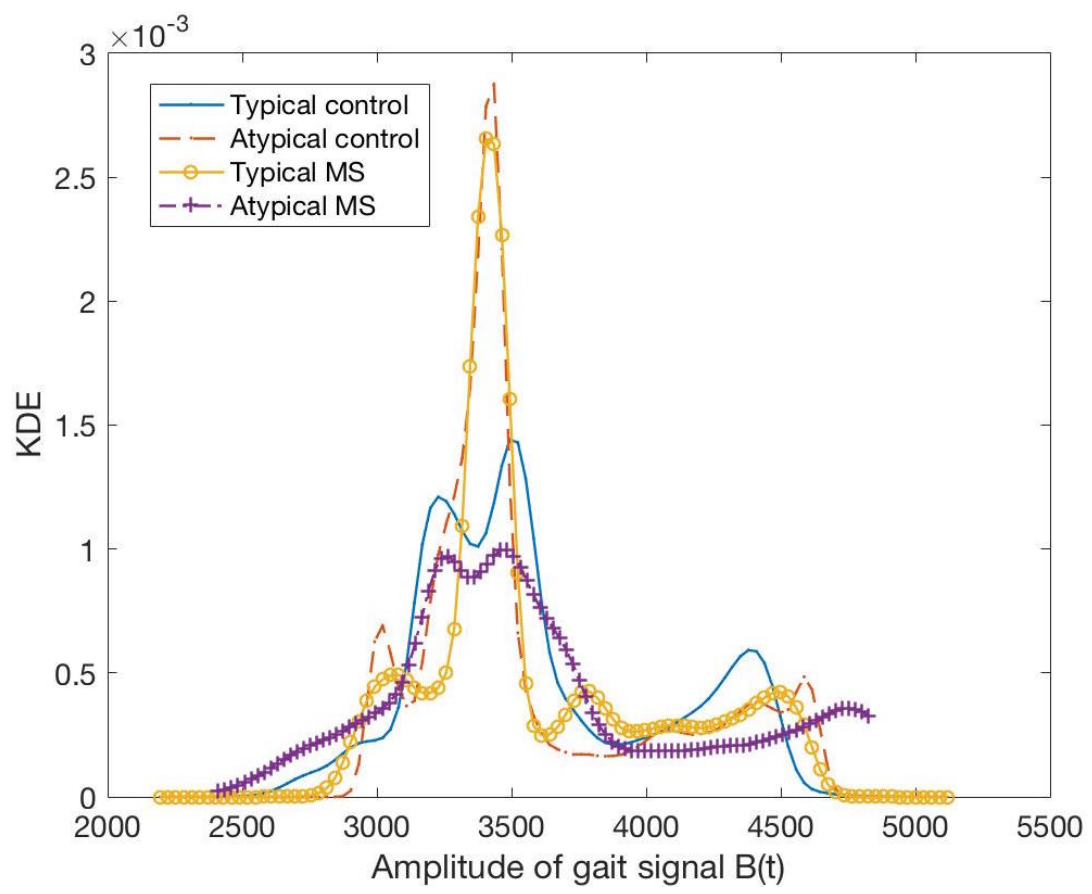

Fig. 7.4: Outliers in control and MS groups: example showing typical and atypical gait densities

It is also worthwhile to note that the gait density estimates were found to be robust and statistically similar to each other $\left(r_{p}=0.82, p<0.0001\right)$, regardless of whether the entire or segments of the 6MWT gait data, corresponding to each minute, were used to estimate the densities, as shown in Fig. 7.5. Thus, the gait KDE is an intrinsic feature. They did, however, change if longitudinal gait data were used.

\subsection{Interpretation of the KDE Peak}

The KDE peak is used as the inertial feature of interest. The statistics of KDE peak are shown in Table 7.1. We observe that although the gait KDE peaks for some MS subjects are comparable to those of controls, the median values for MS subjects are higher than those of controls. 


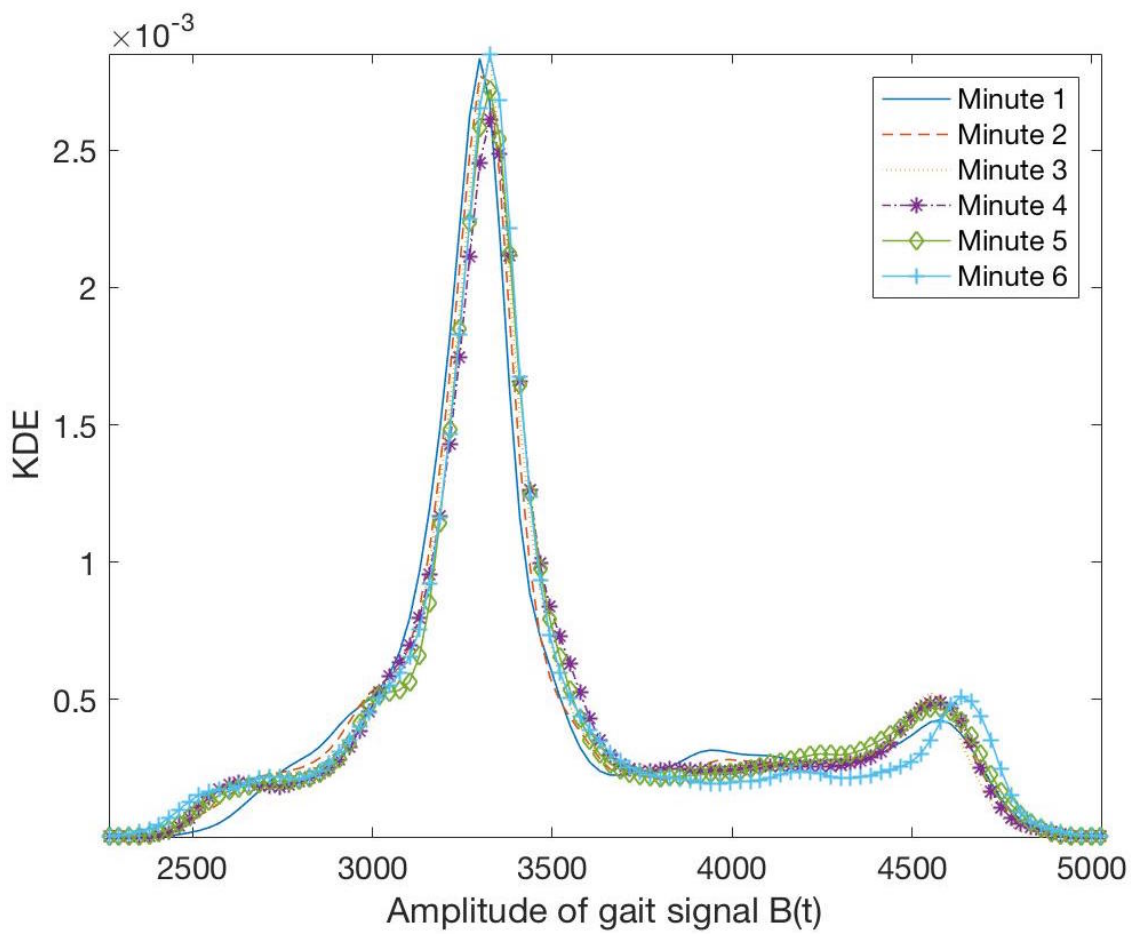

Fig. 7.5: The KDE for each minute of the $6 \mathrm{MWT}$ gait data for an MS subject

Table 7.1: Statistics of the KDE peak

\begin{tabular}{c|c|c|c}
\hline \hline Group & Minimum value & Median value (SD) & Maximum value \\
\hline \hline Controls & 0.0012 & $0.0017(0.0004)$ & 0.0030 \\
\hline Mild MS & 0.0011 & $0.0018(0.0006)$ & 0.0039 \\
\hline Moderate MS & 0.0010 & $0.0024(0.0009)$ & 0.0055 \\
\hline Severe MS & 0.0025 & $0.0073(0.0045)$ & 0.0151 \\
\hline \hline
\end{tabular}

As seen in Chapter 6, the MS subjects who reported to have low disability $(0 \leq$ EDSS $\leq 4$ ) covered 6MWT distance $d$ (plotted again in Fig. 7.6(a)) comparable to those of controls. We divide KDE peaks, denoted as $\rho$ in this work, into three intervals, namely, $0-0.003,0.003-0.0055$, and $0.0055-0.0151$, using maximum values given in Table 7.1. The distances covered by the subjects whose KDE peaks lie in respective intervals are plotted in Fig. 7.6(b). 


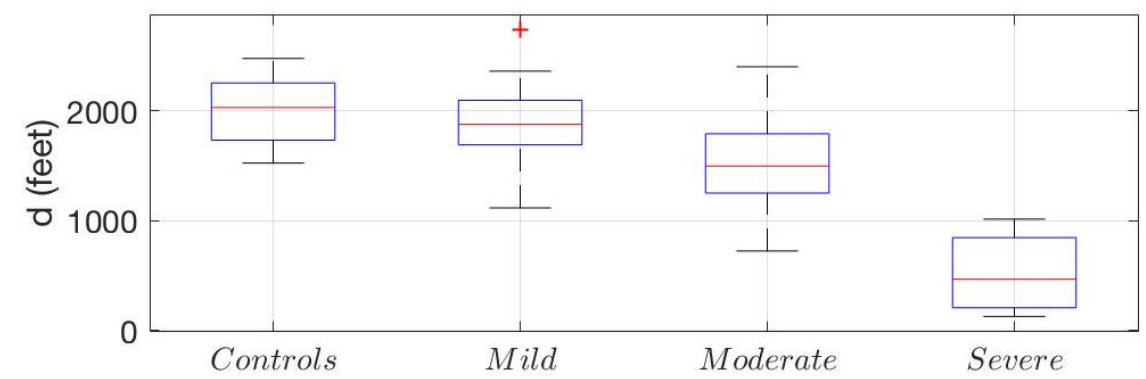

(a) Study groups

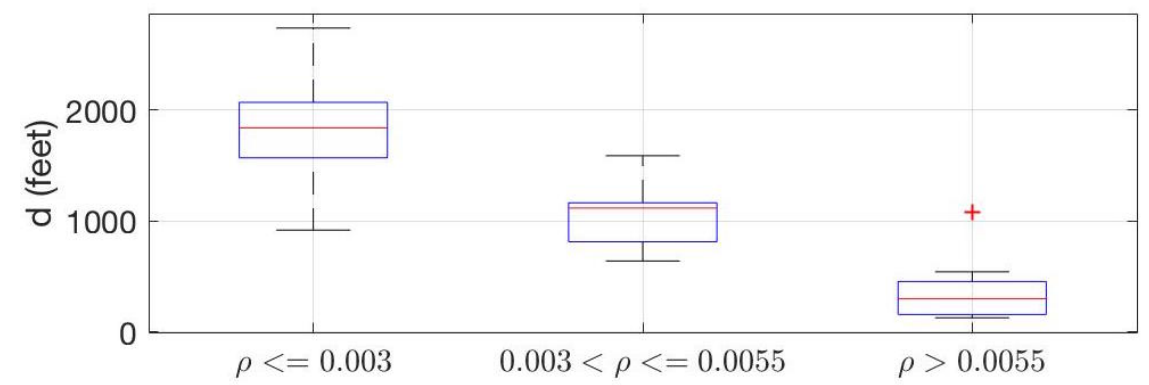

(b) KDE peaks

Fig. 7.6: (a) 6MWT distance $d$ covered by the four study groups, (b) Relationship between intervals of the KDE peaks, $\rho$, and $d\left(r_{s}=-0.788, p=\right.$ $\left.10^{-41}\right)$

Three observations are made.

1. First, KDE peak correlates significantly and negatively with $d,\left(r_{s}=-0.788, p=\right.$ $\left.10^{-41}\right)$.

2. Second, the 6MWT distances and the gait KDE peaks of MS subjects with EDSS up to 4 were comparable to those of control subjects.

3. Third, it suggests that $\rho=0.003$ (for the given data) can be taken as a benchmark for identification of gait impairment.

MS has a variable impact on gait of different subjects and its symptoms vary among individuals. Thus we conclude that if the gait is not affected significantly, the KDE peaks for MS subjects can be as low as those of controls. 


\subsection{Clinical Significance of the KDE Peaks}

\subsubsection{Statistical Analysis}

In order to establish the KDE peak as a useful clinical gait feature, statistical analysis is performed to find its relationships with the clinical data. The relevant clinical sub-scores were identified using (Spearman) correlational analysis and chosen for regression analysis. The corrected significance level was found to be 0.0002 . The step-wise linear regression models (LRMs) $\mathcal{M}_{X}^{Y}$ were then fitted with

- $X \in\{$ Significantly correlated clinical sub-scores $\}$

- $Y \in\{6 \mathrm{MWT}$ speed (feet/s or fps), KDE peak $\}$

The adjusted $R^{2}$ values from LRMs are given in Table 7.2.

Table 7.2: The adjusted $R^{2}$ from step-wise LRMs

\begin{tabular}{l|c|c}
\hline \hline Clinical category $^{1}$ & 6MWT speed & KDE peak \\
\hline \hline SF36 (Q4b, 4c, 4e, 4f, 4g, 4h, 4i, 4j, total) & 56.0 & 54.4 \\
\hline Daily activities & 57.7 & 81.2 \\
\hline FSS (pyramidal, cerebellar, bladder, cerebral) & 62.5 & 39.3 \\
\hline Physical fatigue & 63.6 & 30.8 \\
\hline MFIS (Q4, 6-10, 13, 14, 17, 18, 20, 21, total) & 65.2 & 36.8 \\
\hline EDSS & 67.1 & 45.1 \\
\hline Cognitive/concentration & 68.9 & 38.4 \\
\hline PDDS & 75.0 & 51.6 \\
\hline MSWS (Q1-12, total) & 75.4 & 53.9 \\
\hline Balance & 76.7 & 60.0 \\
\hline Weakness & 77.8 & 53.9 \\
\hline Mobility, sensory, pain, tremor & 78.4 & 57.2 \\
\hline \hline
\end{tabular}

${ }^{1}$ From Table 3.1. For each category, only significantly correlated sub-scores are used. The rows are arranged in increasing order of the adjusted $R^{2}$ values for the $6 \mathrm{MWT}$ speed. 
The KDE peaks show strong relationships with various types of clinical data, although some of these relationships are weaker than others. The relationships of each clinical category to KDE peak are discussed in the next section.

\subsubsection{Results and Discussions}

In this section, the significantly correlated sub-scores are plotted against KDE peak. For each plot, the $x$-axis represents the clinical scores reported using ordered categories. If the number of ordered categories are more 12, the three intervals of $\rho$, shown above, are used as the $x$-axis.

1. As seen previously, the KDE peaks of MS subjects, assigned an EDSS score of up to 4, overlap with those controls. The regressions results confirm that EDSS has a weak but statistically significant relationship with the KDE peak, as shown in Fig. 7.7.

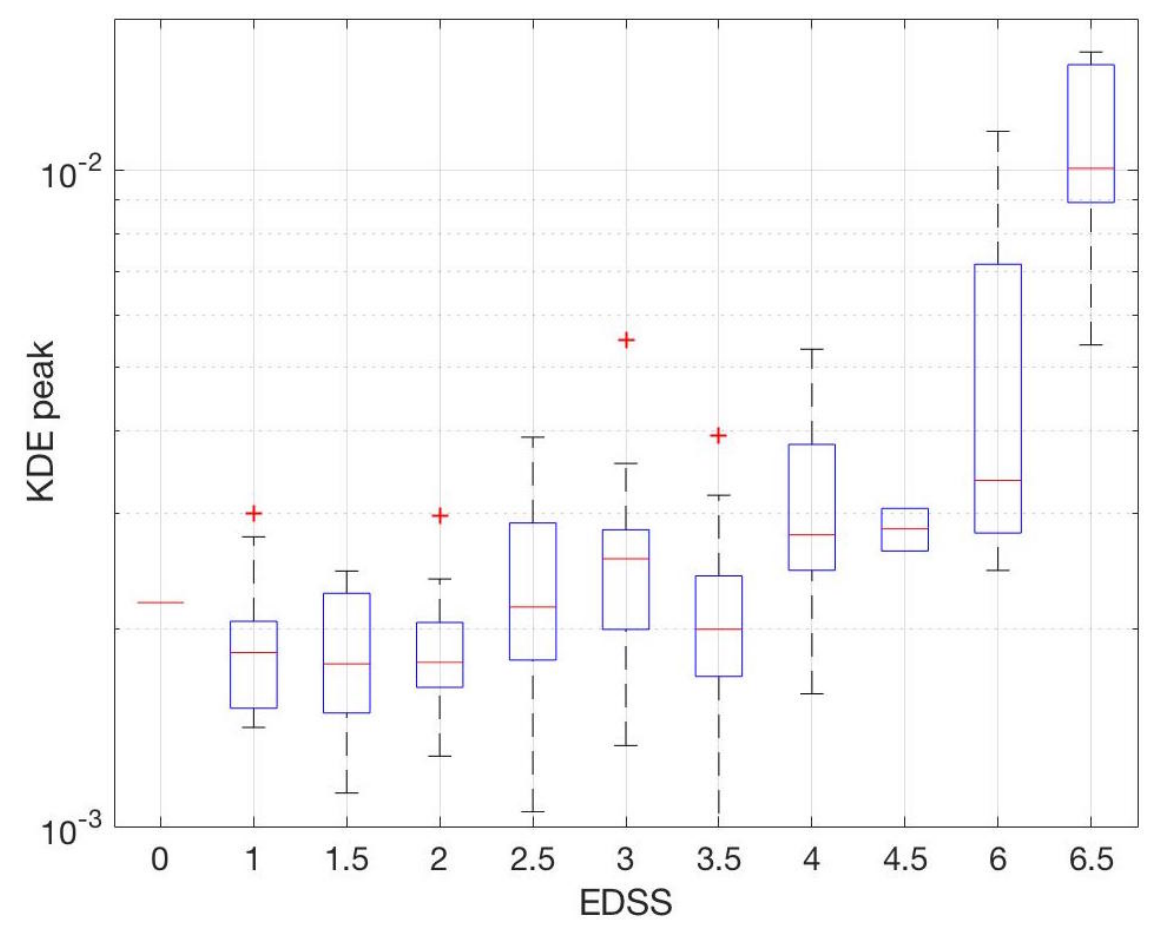

Fig. 7.7: Relationship between EDSS and the KDE peak 
2. Statistical analysis shows that four FSS components, namely, pyramidal, cerebellar, bladder, and cerebral, have statistically significant relationships to the KDE peak, as shown in Fig. 7.8. The weak relationship of the KDE peak with the cerebral function impairment is its divergent validity.

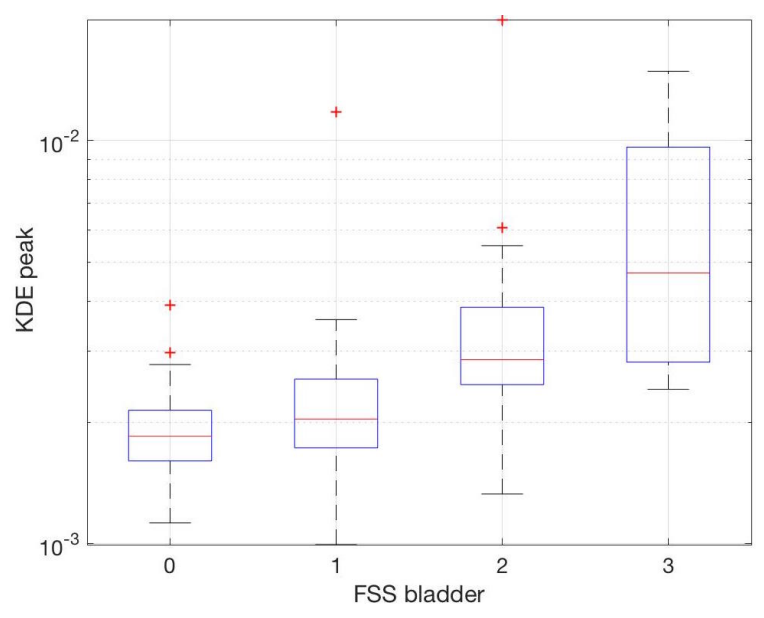

(a) FSS bladder

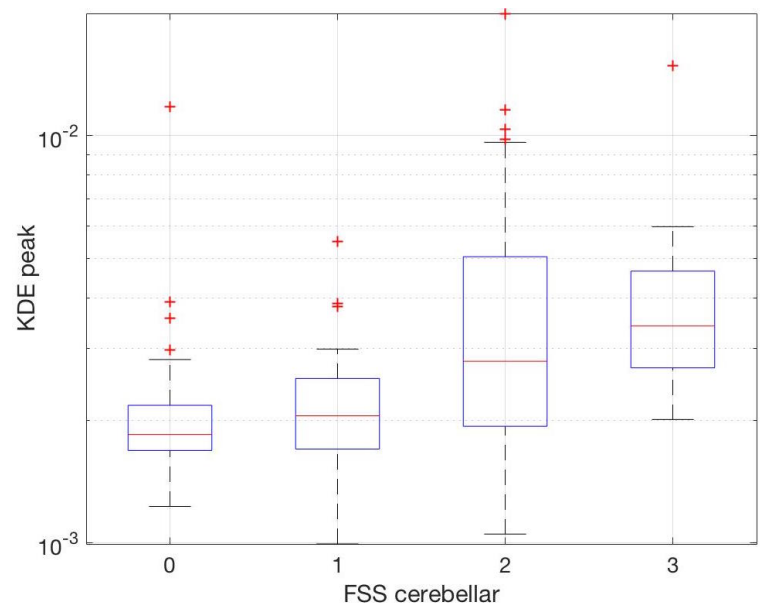

(c) FSS cerebellar

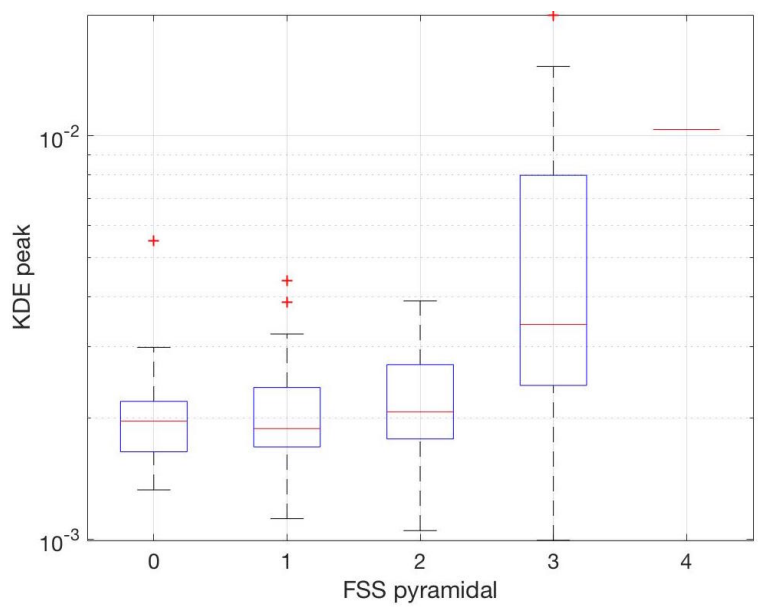

(b) FSS pyramidal

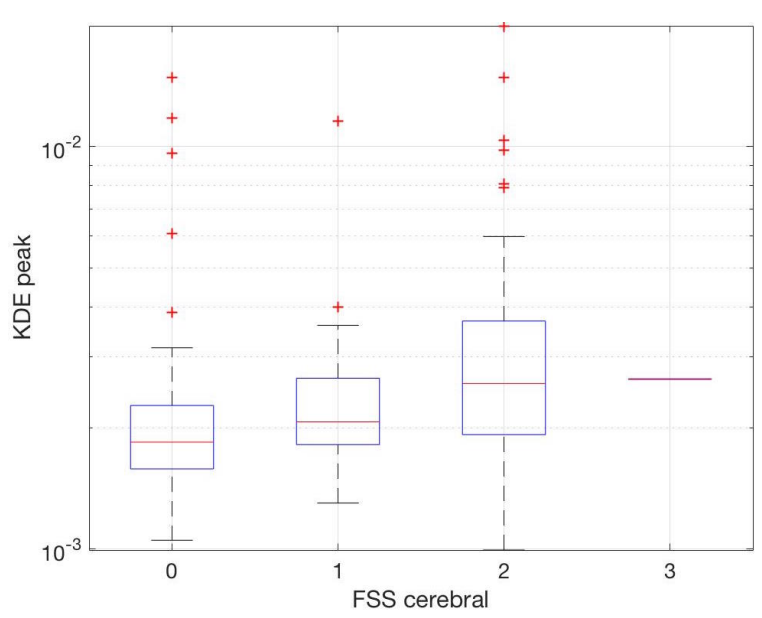

(d) FSS cerebral

Fig. 7.8: Relationships between FSS components and the KDE peak 
3. PDDS also shows robust relationships to the KDE peak, shown in Fig. 7.9.

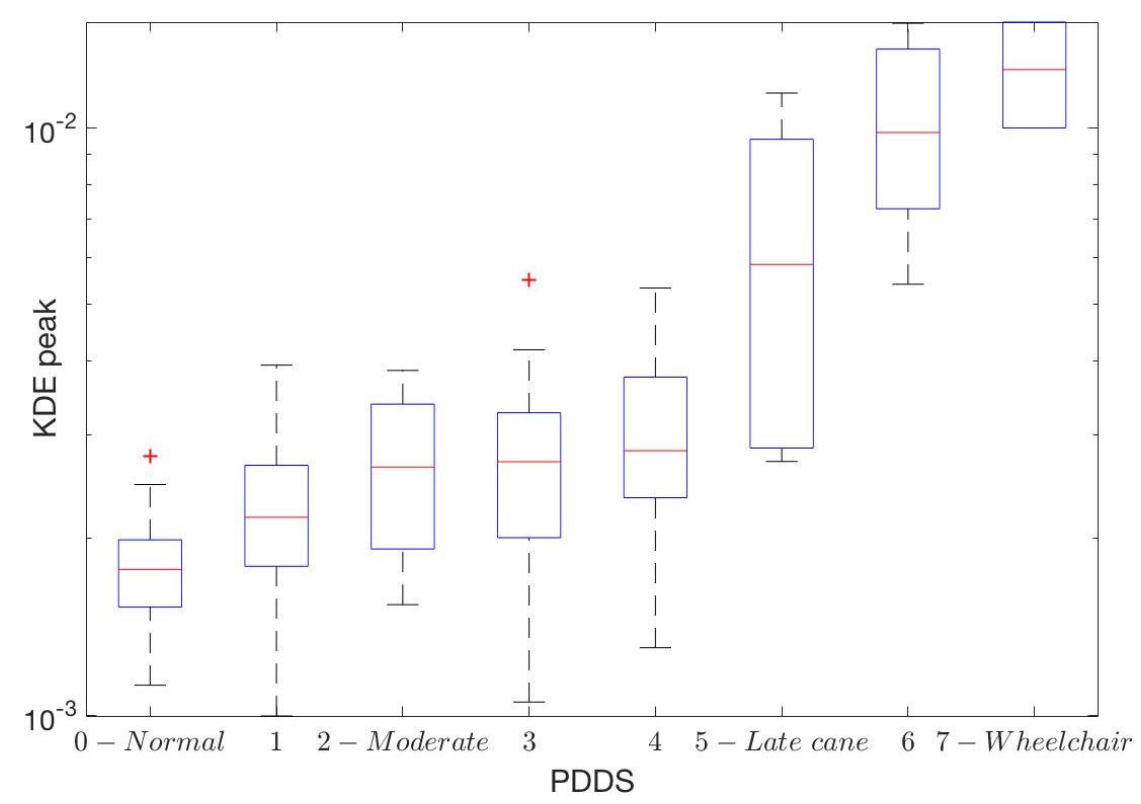

Fig. 7.9: Relationship between PDDS and the KDE peak

4. All MSWS scores have strong relationships with the KDE peak. Two MSWS components related to standing and balance, (Q4, 5), are shown in Fig. 7.10. Thus, higher KDE peaks indicate balance problems.

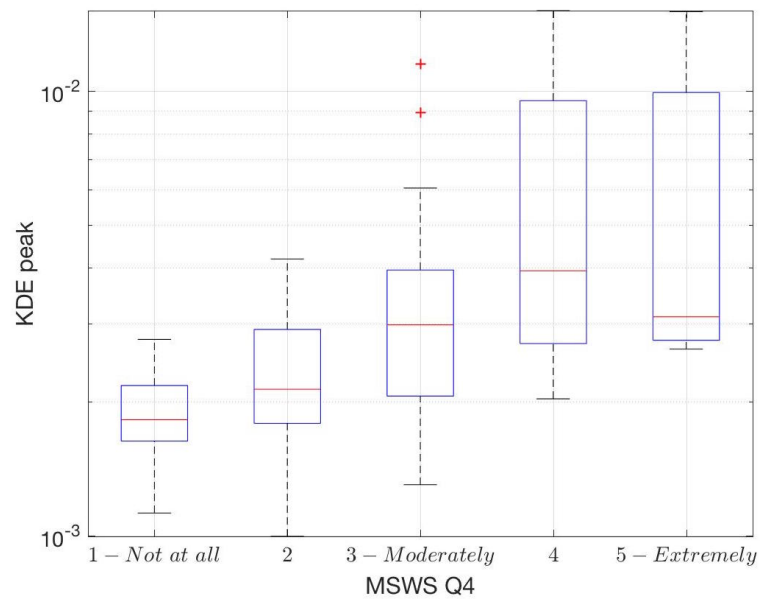

(a) "Standing when doing things difficult"

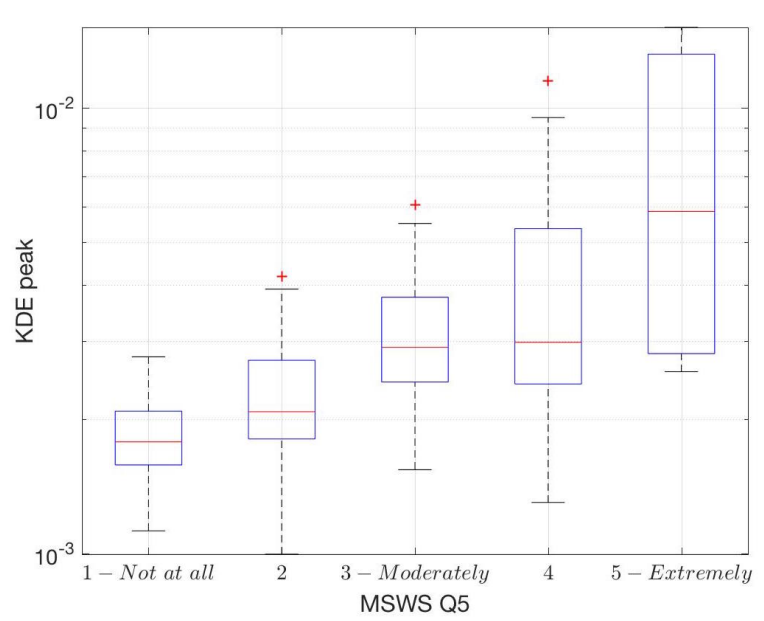

(b) "Limited balance when standing or walking"

Fig. 7.10: Relationships between MSWS components and the KDE peak 
5. As shown in Fig. 7.11, each of mobility, sensory, and pain showed robust relationships to the KDE peak. Tremor/loss of coordination demonstrated weak but statistically significant relationships to the KDE peak.

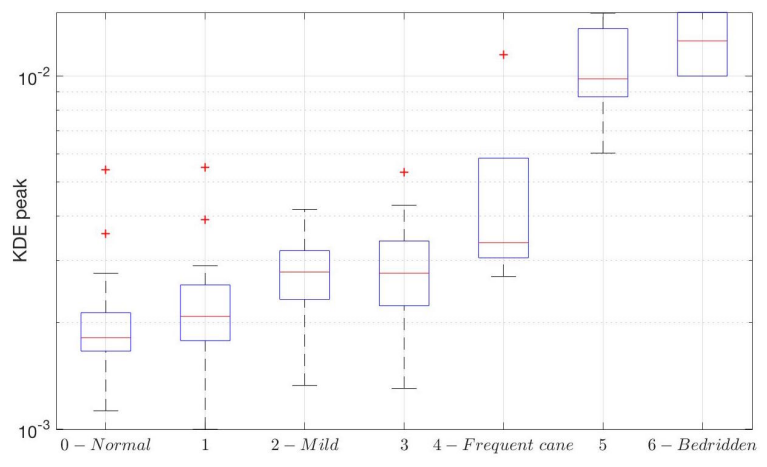

(a) Mobility

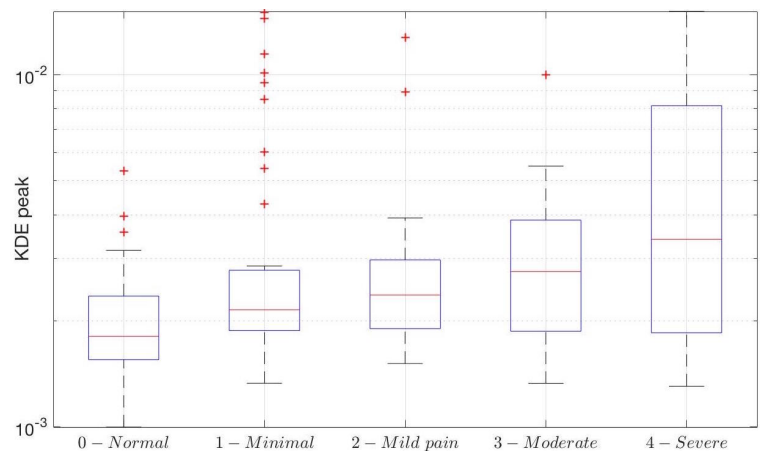

(c) Pain

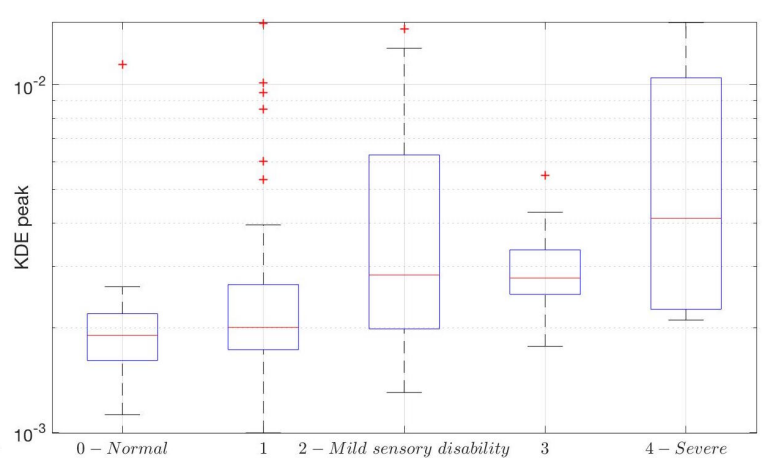

(b) Sensory

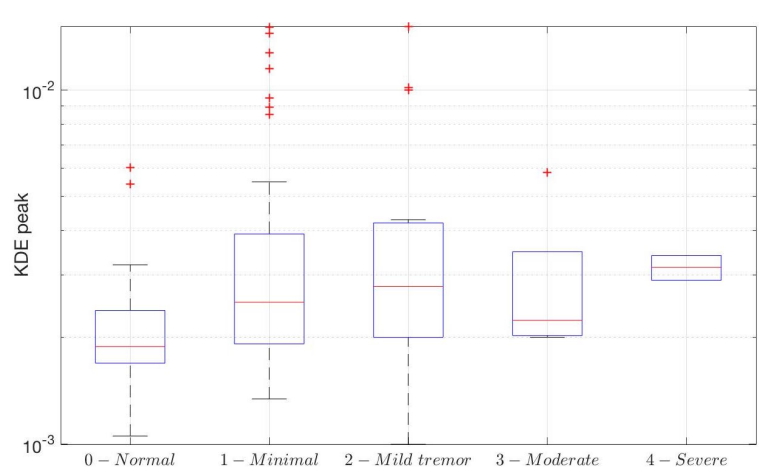

(d) Tremor

Fig. 7.11: Relationships between performance scales and the KDE peak

6. Fig. 7.12 shows that instrumental activities of daily living (IADL) exhibit strong relationships to KDE peak. Clearly, the gait of subjects who reported problems in carrying out day-to-day activities had higher KDE peaks. 


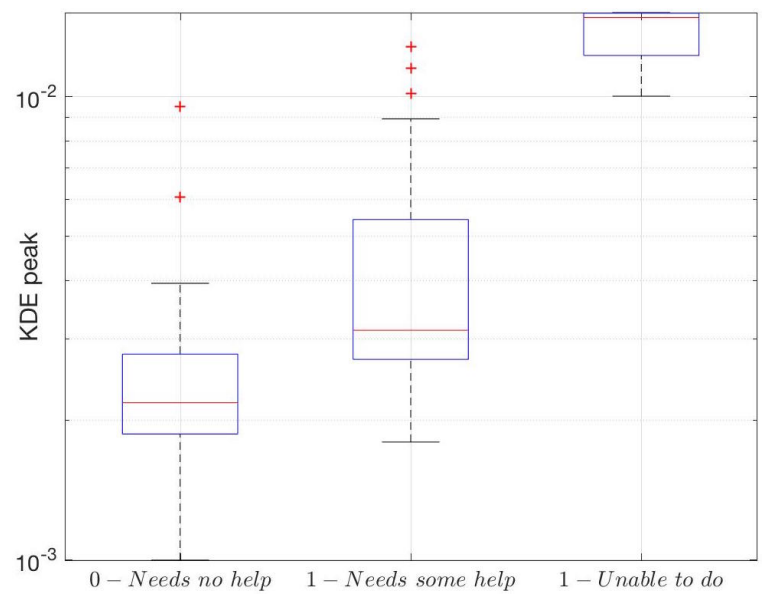

(a) Difficulty doing housework

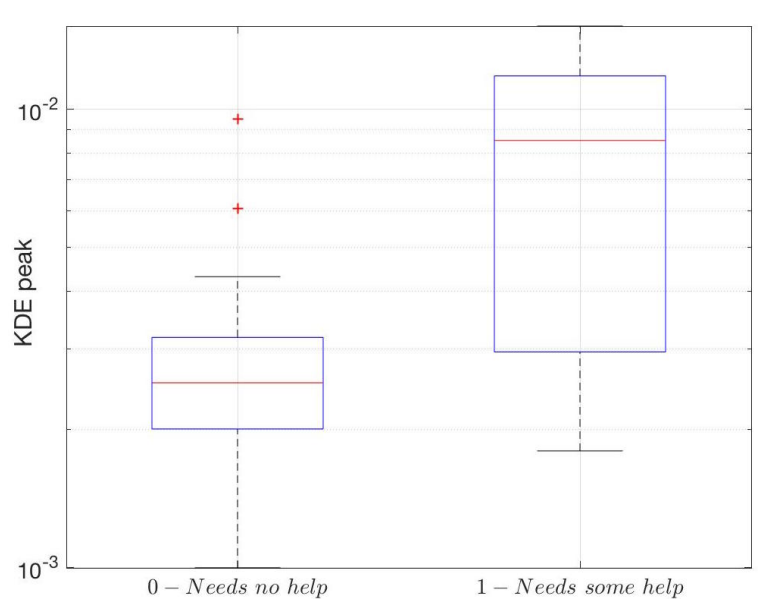

(c) Difficulty preparing meals

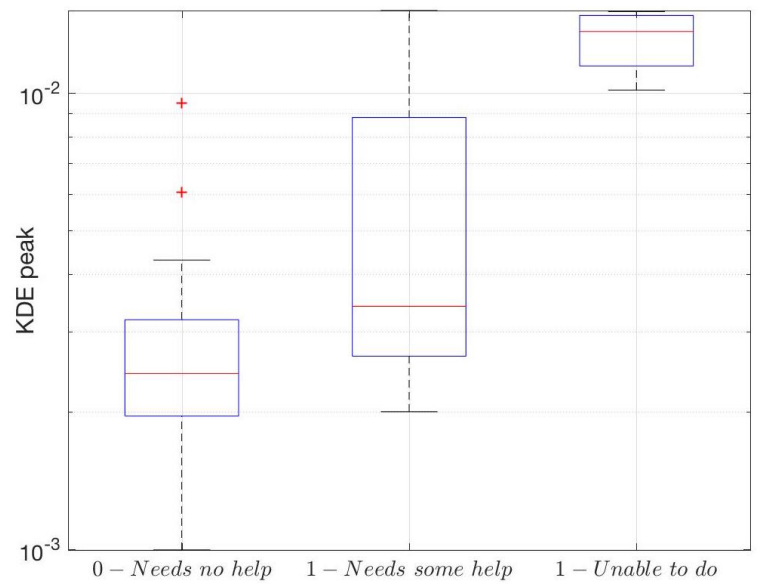

(e) Difficulty getting beyond walking distance

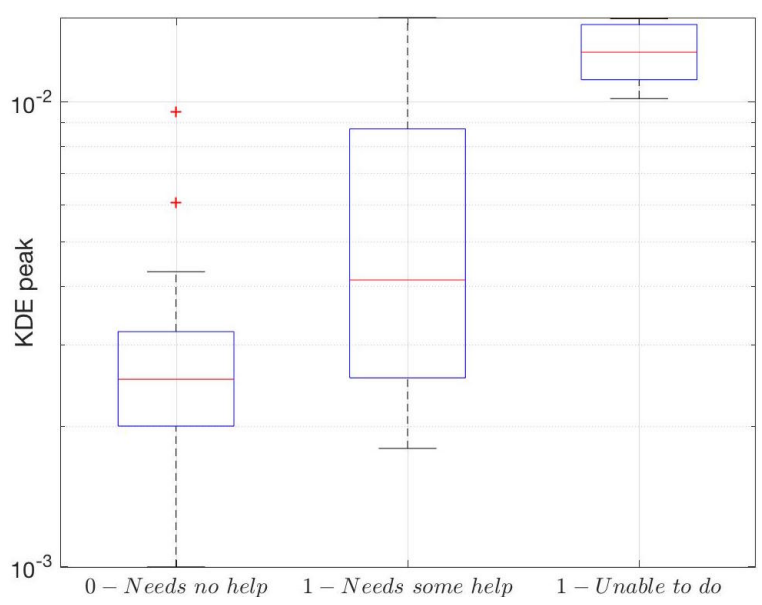

(b) Difficulty doing laundry

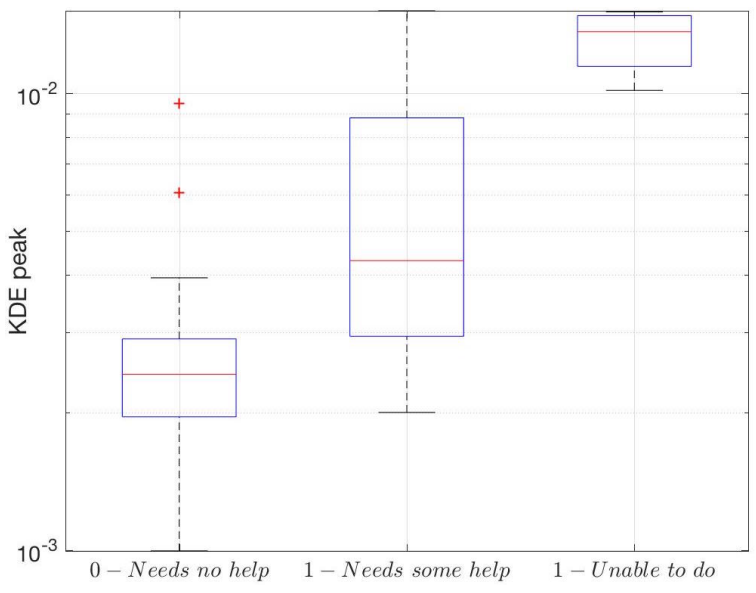

(d) Difficulty doing grocery shopping

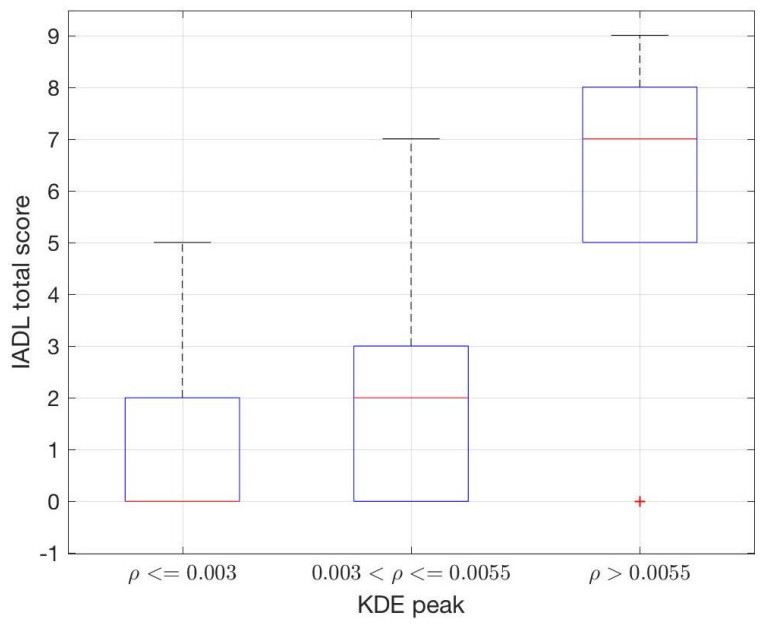

(f) Total IADL score

Fig. 7.12: Relationships between IADLs and the KDE peak 
7. SF36 Q4 assesses limitations in doing activities on a typical day due to health [10]. It is striking that SF36 components from Q4 as well as total SF36 show significant correlations with the KDE peak, as shown in Figs. 7.13, 7.14. The KDE peaks were found to be greater than 0.003 for subjects who reported that motor-related fatigue had a higher impact on their lives.

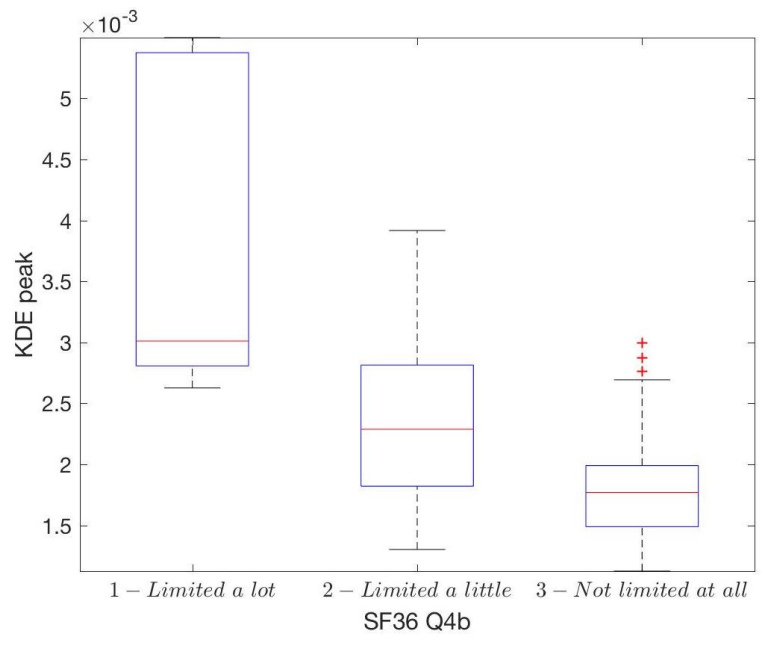

(a) "Moderate activities"

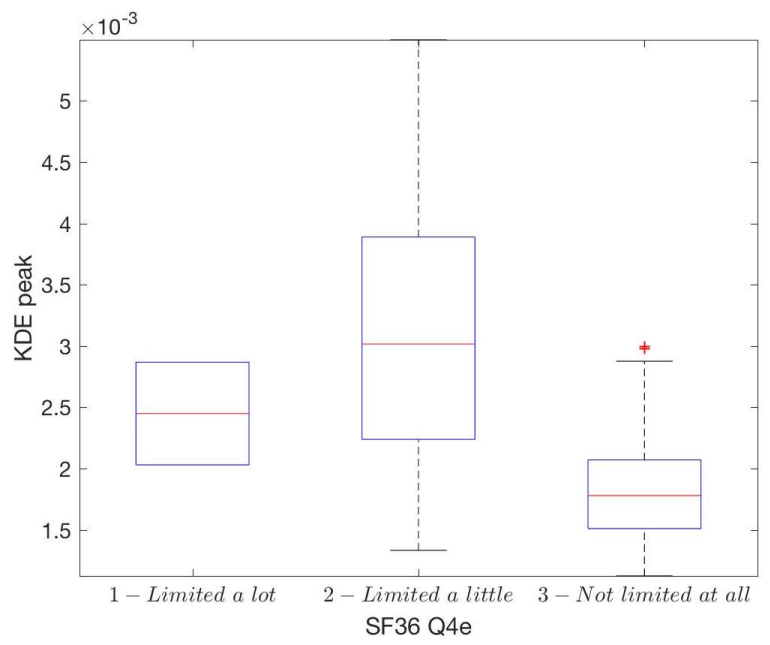

(c) "Climbing one flight of stairs"

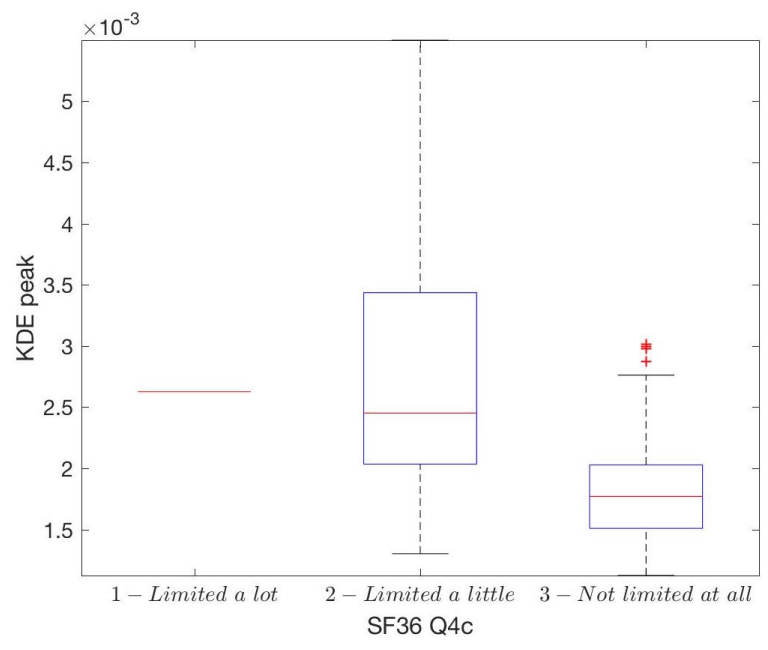

(b) "Carrying groceries"

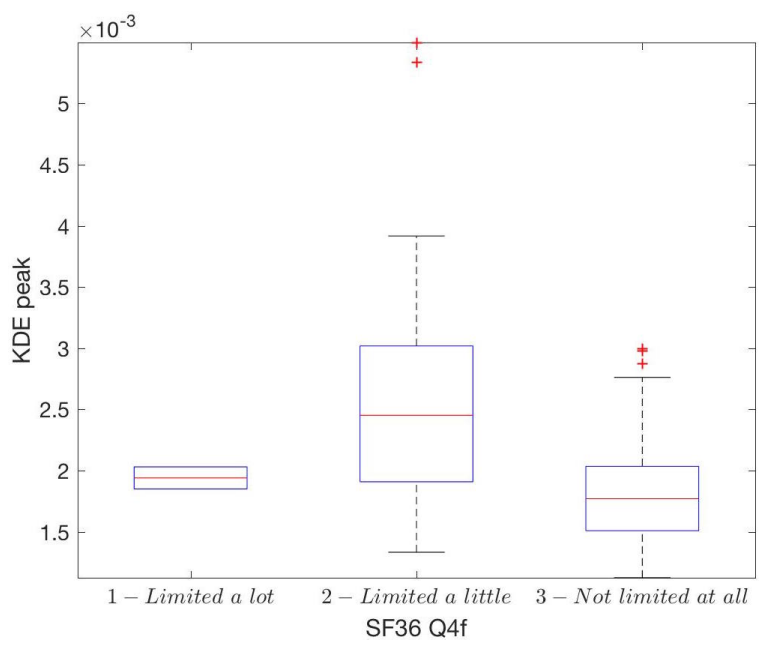

(d) "Bending, kneeling, or stooping"

Fig. 7.13: Relationships between SF36 components (Q4 b, c, e, f) and the KDE peaks 


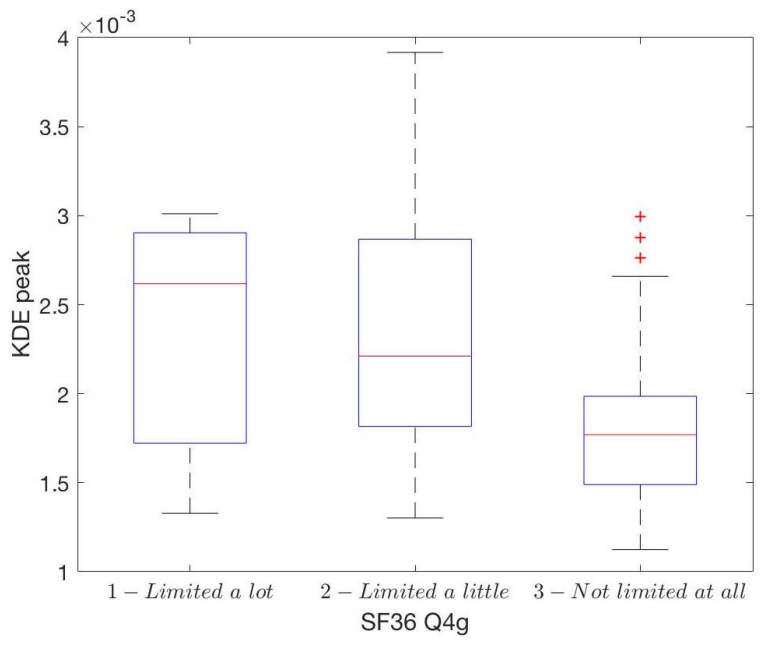

(a) "Walking more than a mile"

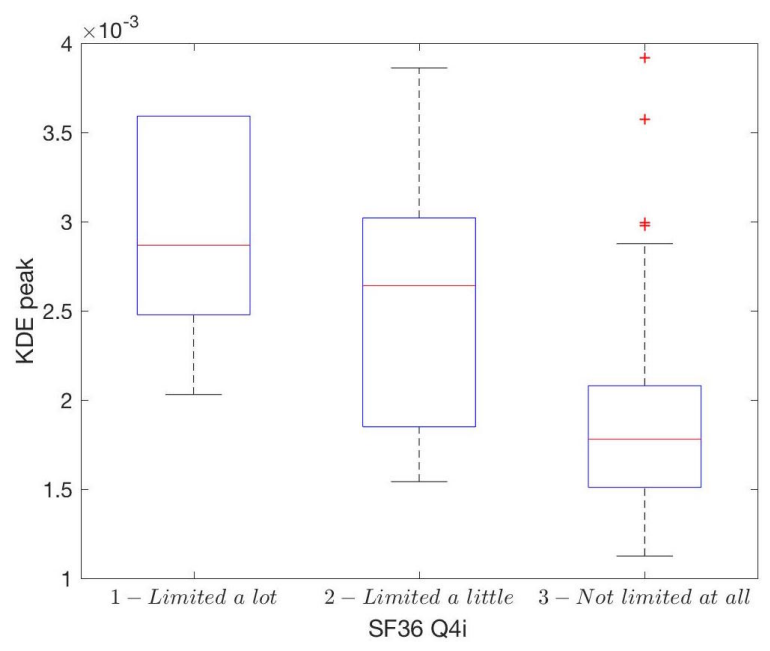

(c) "Walking one block"

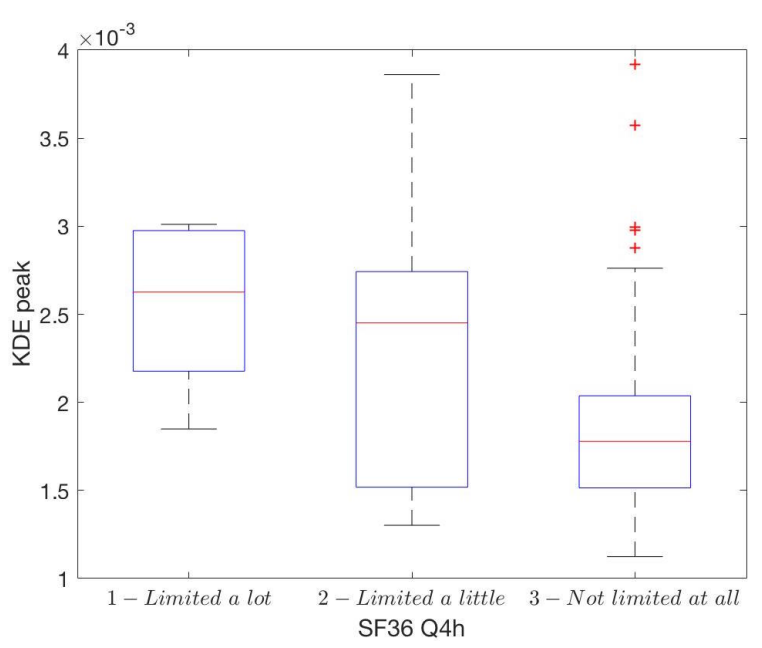

(b) "Walking several blocks"

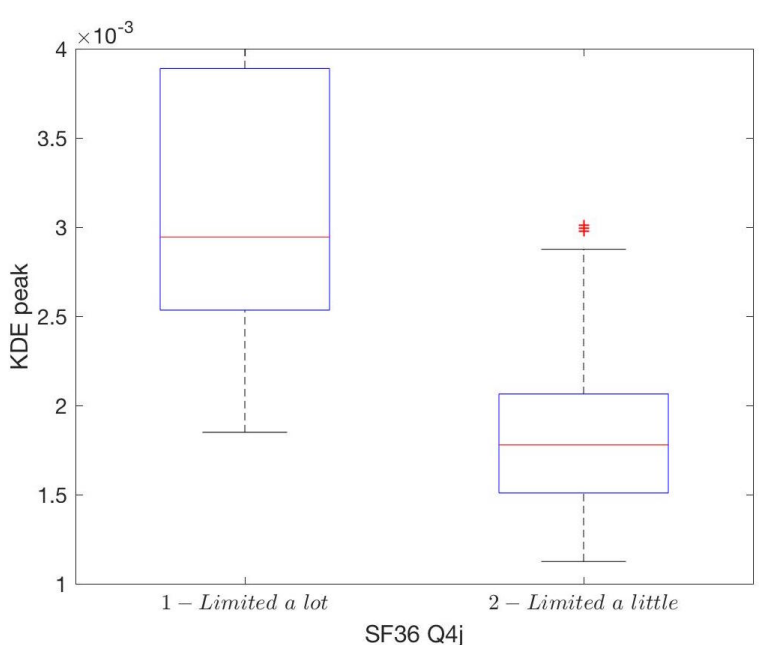

(d) "Bathing or dressing"

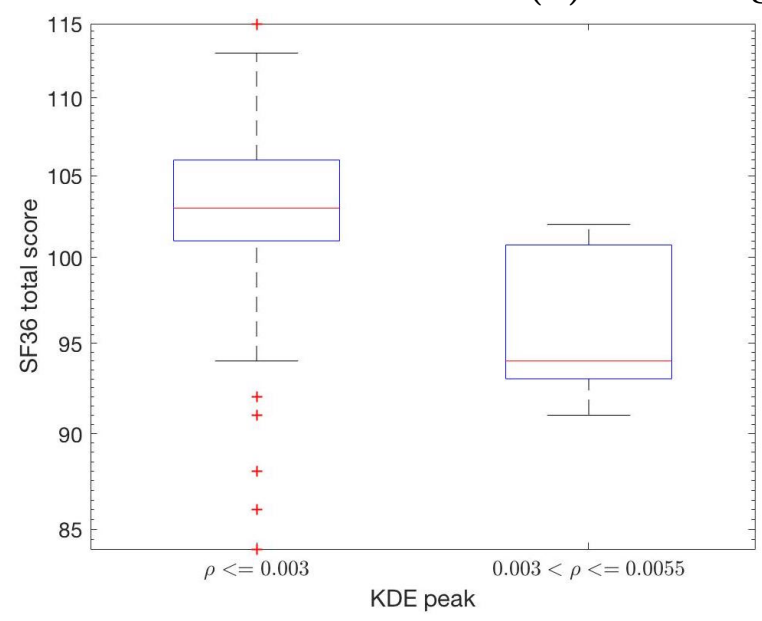

(e) SF36 total score

Fig. 7.14: Relationships between SF36 components (Q4 g-j, total score) and the KDE peak 
8. Numerous MFIS components as well as total MFIS show significant correlation to the KDE peaks, as shown in Fig. 7.16. The KDE peaks were found to be greater than 0.003 for subjects who reported that motor-related fatigue had a higher impact on their daily lives.

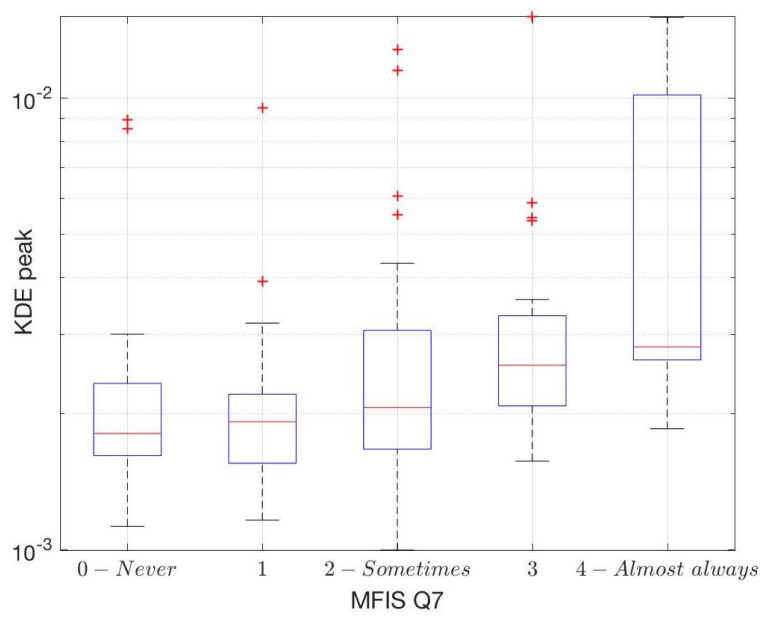

(a) "Less motivated for physical effort"

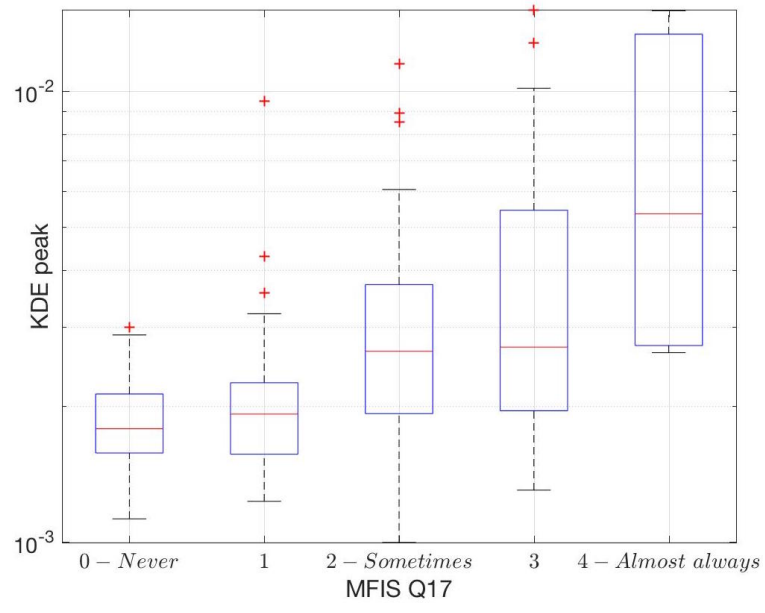

(c) "Less able to do physical tasks"

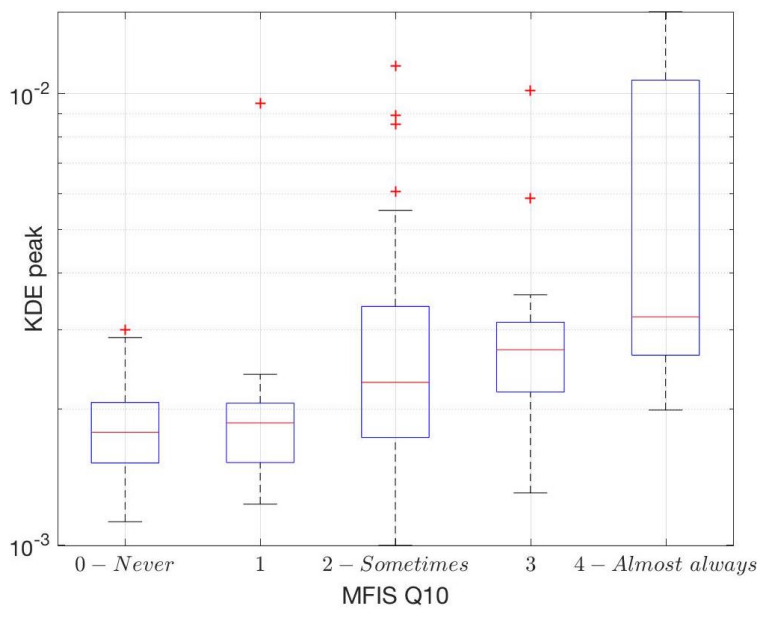

(b) "Trouble maintaining physical effort"

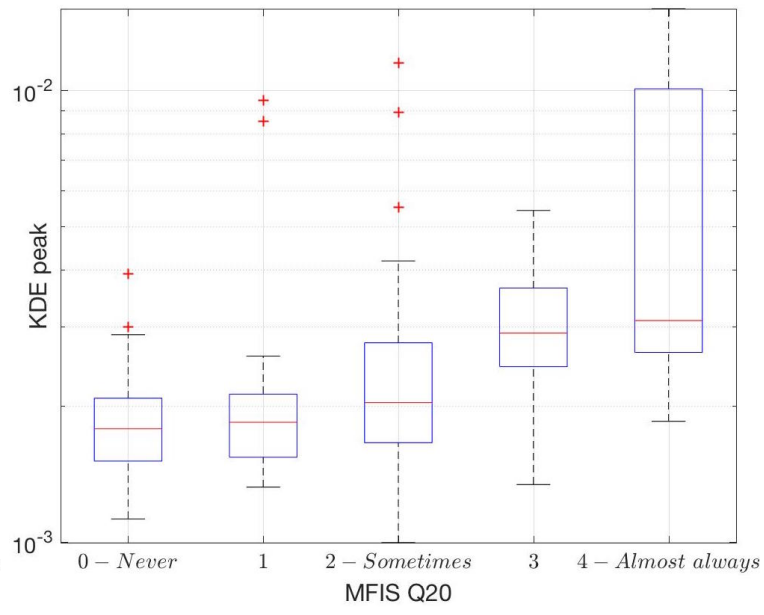

(d) "Limited my physical activities"

Fig. 7.15: Relationships between MFIS components (Q7, 10, 17, 20) and the KDE peak 


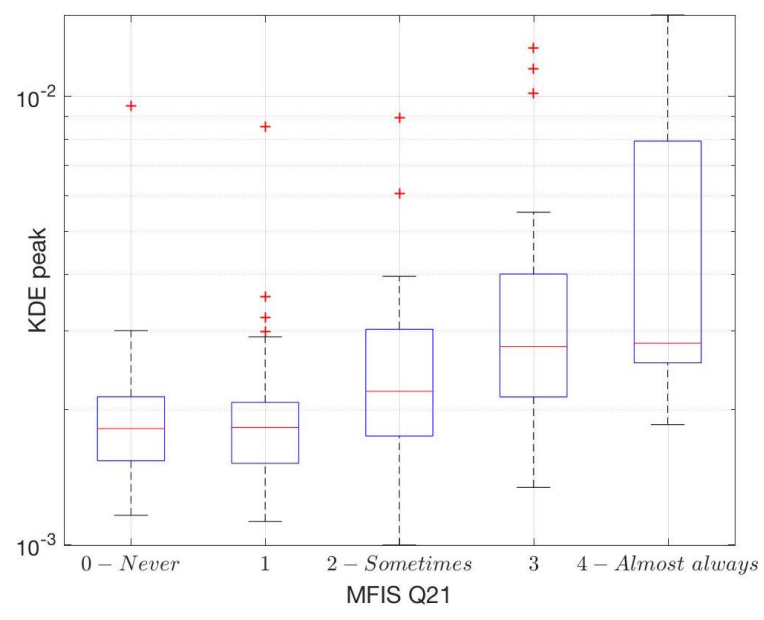

(a) "Needed to rest more"

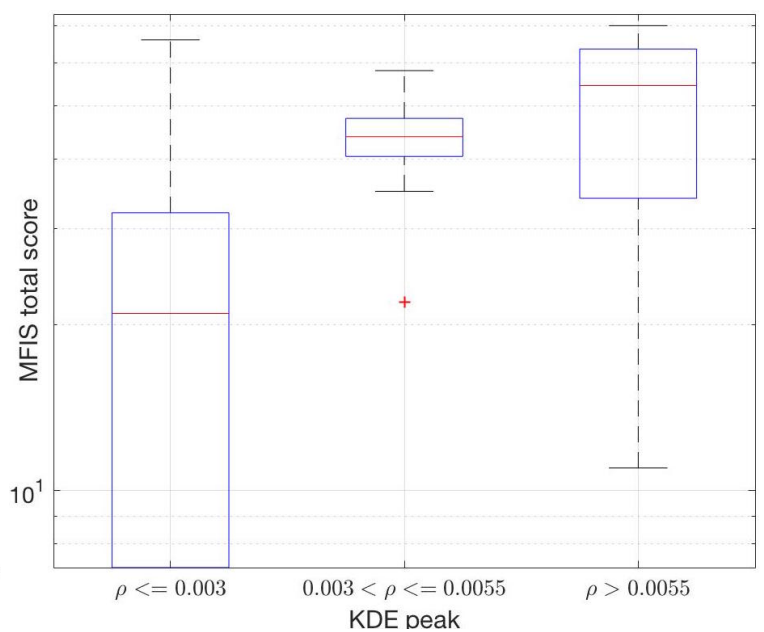

(b) Total MFIS score

Fig. 7.16: Relationships of KDE peak to MFIS Q7 and its total score

Relationship of KDE peak to four aspects of MS disability. As shown in Chapter 6, the 6MWT speed has strong relationships with various disability domains of MS as compared to previously reported inertial measures. Table 7.2 shows that the KDE peaks also show robust relationships to these aspects. These relationships are non-uniform and weaker than those for $6 \mathrm{MWT}$ speed. The adjusted $R^{2}$ for the KDE peaks is close to 60 for weakness and balance each, while it is almost half for both physical fatigue and cognitive/concentration.

The percentage contributions of these disability aspects to the KDE peaks and 6MWT speed are given in Table 7.3 and plotted in Fig. 7.17. Specifically, a high value of the KDE peak is an indicator of weakness and balance problems, making it the strongest overall measure of walking impairment, better than some of the other inertial measures [99]. This is also evident from individual sub-scores shown above, such as MSWS Q5. Thus, the peak value of the KDE could be used alongside the gait speed to supplement weakness and balance deterioration information. 
Table 7.3: The \% adjusted $R^{2}$ for four aspects of MS disability

\begin{tabular}{c|c|c|c|c}
\hline \hline Gait feature & Weakness & Physical fatigue & Balance & Cognitive/concentration \\
\hline \hline KDE peak & 29.4 & 16.8 & 32.8 & 21.0 \\
\hline 6MWT speed (fps) & 27.1 & 22.2 & 26.7 & 24.0 \\
\hline \hline
\end{tabular}

The contribution of physical fatigue to the KDE peak is the smallest, $4 \%$ smaller than that of cognition/concentration impairment. The KDE peak values not only distinguish between various disability groups but also track with self-reported functional impairment and balance difficulty. Weakness and balance problems put people with MS at high risk for falling. Thus, the KDE peaks could possibly be used to identify subjects who are at high risk for falls.

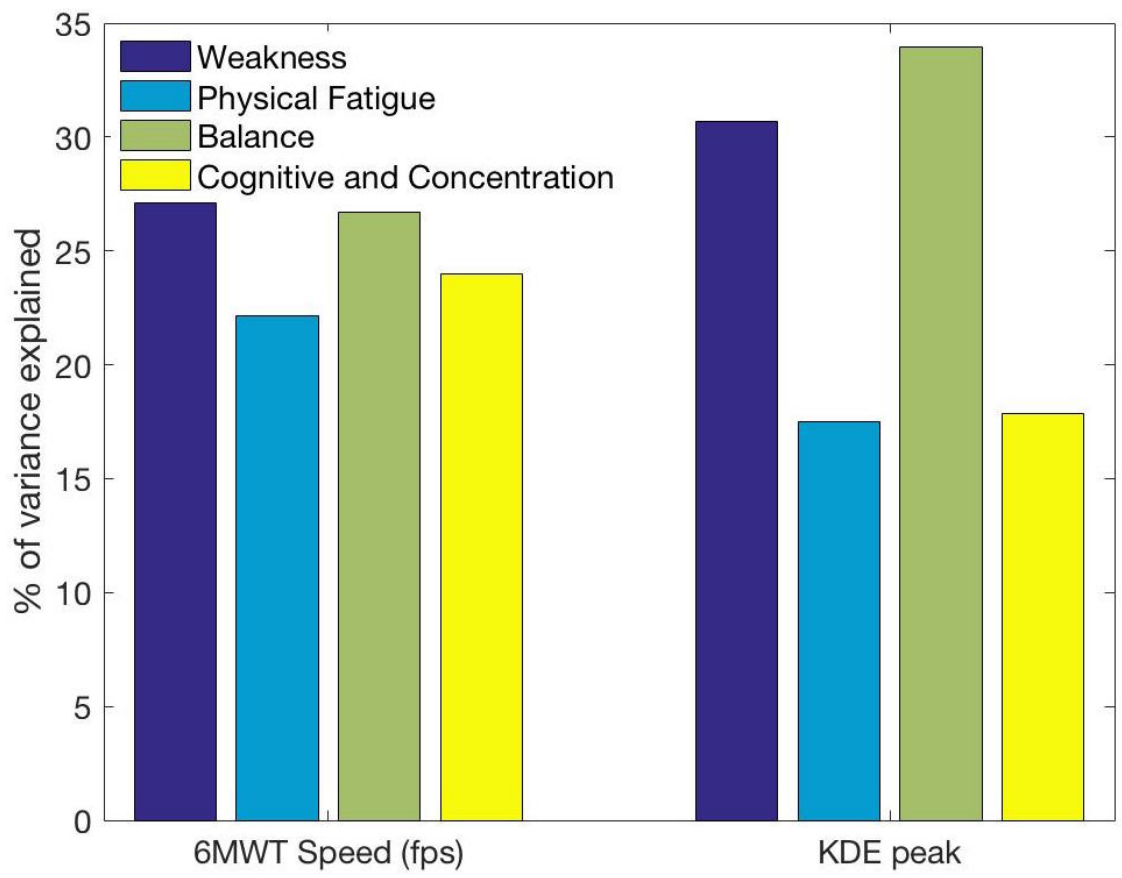

Fig. 7.17: \% of variance explained by the four aspects of MS disability 


\subsection{Summary}

The peak value of the gait KDE is an excellent measure of weakness and balance difficulty, the two most prominent causes of gait impairment. The gait density estimates did not change when computed separately for each of the six-minutes $\left(r_{s}=0.82, p<0.0001\right)$, indicating that the gait density is an intrinsic feature. They did, however, change if longitudinal gait data were used, with changes being correlated to the changes in clinical symptoms.

The gait KDE peaks for MS subjects in the low disability levels might be comparable to those of control subjects. There is also less variability in the gait signal amplitude for MS subjects, which means the KDEs are concentrated around smaller range of values, leading to high peaks. Thus peak value above a threshold is, indeed, an evidence of MS-related walk deterioration, as supported by the clinical data. Using the relationships of the clinical data to the KDE peak, we conclude that a threshold of $\mathrm{KDE}$ peak $=\mathbf{0 . 0 0 3}$ (for the given data) could be used as a clinical anchor. In cases where the KDE peaks are found to be above the threshold, relevant in-depth subjective assessments could be performed, thus reducing the overall burden of those questionnaires. The threshold can easily be determined for any data-set in a similar way. Some of the benefits of the KDE peak are:

1. The KDE peak explains over $50 \%$ of the variance in clinical outcomes measuring weakness and balance problems, making them the strongest overall measures of walking impairment, better than some of the other inertial measures [99].

2. Computation of other commonly used gait variables such as stride time SD [114], dynamic time warping (DTW) scores [17], gait phase, stride time [15], etc., depend on accurate identification of gait cycles, which is a computationally ex- 
pensive and noise-sensitive process. The KDE peak is most convenient from a processing standpoint, as it is distribution based and does not require segmentation of gait data into individual gait cycles.

3. The density estimates were found to be robust and statistically similar to each other, regardless of whether the entire or a subset of the 6MWT gait data were used to determine the KDEs. This would be beneficial in scenarios where resources (storage/power) are limited, e.g., when computing the KDE on wearable BSN nodes for longitudinal gait monitoring. The density estimates of the gait data can be computed periodically so that an equivalent but small dataset be stored on nodes. 


\section{Chapter 8}

\section{GAIT CYCLE EVENT-SPECIFIC SPECTRAL FEATURES}

This chapter studies the spectral features obtained from a gait cycle and its phases. The relationships of these features with the clinical data verify the hypothesis that phase-specific gait features indicate different types of MS-induced disabilities. The work presented in this chapter was published in [117].

\subsection{Motivation}

Features obtained using the gait time series data corresponding to events in a typical gait cycle may provide specific information regarding the variable impact of the disease on various gait components. Such features can also be used as markers of balance, stability, fall risk, etc. Identifying important parts of a gait cycle is advantageous from resource point of view since processing the entire gait cycle is more computationally intensive. Thus we extract spectral features using parts of gait cycles corresponding to three important phases, namely, mid-swing to HS (deceleration phase), HS to TO (foot-flat), and TO to mid-swing (acceleration phase), shown in 
Fig. 4.2.

We obtain the following results using phase-specific spectral features:

- Most of the information regarding health is contained in the TO to mid-swing and mid-swing to HS phases.

- The TO to mid-swing phase is the most indicative of functional and cognitive health status in MS. In fact, it is the only phase that measures the impacts of motor-fatigue on functional, physical, and cognitive components.

- The mid-swing to HS phase is specific to motor problems.

- Foot-flat showed statistically strong and significant relationships to instrumental activities of daily living.

The adjusted $R^{2}$ obtained using step-wise linear regression models, relating spectral features to clinical data, corresponding to the three phases are reported. In order to show the clinical utility of an event-specific analysis, the regression models, showing the relationships between clinical data and the spectral features obtained from an entire gait cycle, are also generated.

\subsection{Extraction of Gait Phases}

We segment each gait cycle into three phases, namely, mid-swing to HS, HS to TO, and TO to mid-swing. Recall from Fig. 4.3, the peaks of the signal $B$ correspond to the mid-swing, whereas the troughs before and after mid-swing represent TO and HS, respectively. Since the stride time varies from cycle to cycle, each gait cycle, $C(t)$, is re-sampled to have 300 samples $(\approx 2.34 \mathrm{~s})$, as shown in Fig. 8.1, and segmented into three windows, each comprising 100 samples. The resampling of a gait cycle into 
300 samples works well for our data since it allows the three gait phases of interest to naturally fall into one of the three windows. The first window represents mid-swing to HS phase, the second corresponds to foot-flat, and the third represents TO to HS transition of the gait cycle.

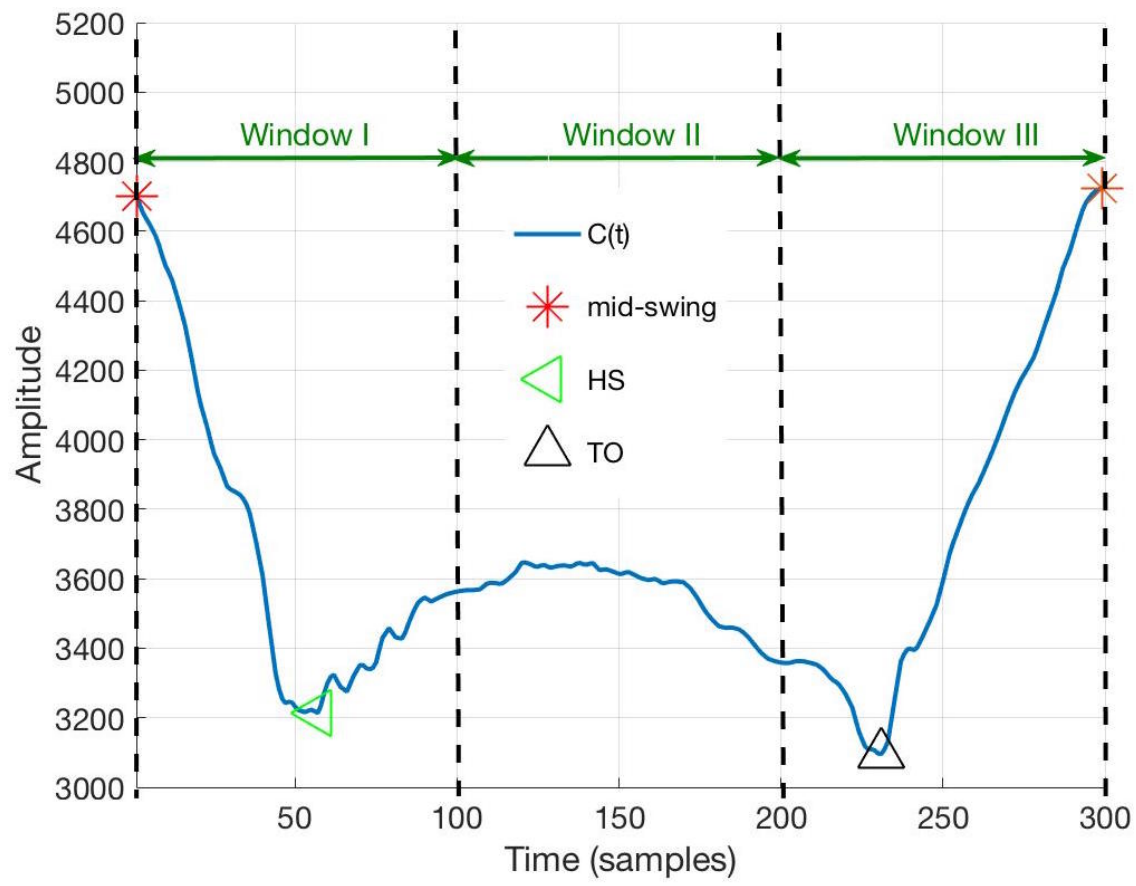

Fig. 8.1: A gait cycle re-sampled to have 300 samples and divided into three windows. The windows (approximately) represent the three gait phases, namely, mid-swing to HS, HS to TO, and TO to mid-swing

It should be noted that above segmentation is not perfect, for example, the 100th sample after mid-swing extends beyond the HS. To segment gait cycles into respective phases based on the actual local locations of HS and TO, the procedure given in [15] can be used. However, in the presence of multiple troughs near HS and TO, it is difficult to identify the exact locations of these events. Our procedure is not simple, but it also guarantees that the first window completely captures the end of HS. Similarly, it makes sure that the third window starts before or at the TO. 


\subsection{Mean Magnitude Spectral Density}

From the Hilbert spectrum given in (2.11), a two-dimensional feature, known as the marginal spectrum or magnitude spectral density $h(f)$ [26], is obtained. If $f_{\max }$ is the maximum frequency component present in the underlying signal, then

$$
h(f)=\int_{t} H(f, t) d t, f \leq f_{\max } .
$$

The marginal spectrum is a measure of the total magnitude contribution from a frequency component present in the data. Note that, unlike the Fourier transform, a non-zero value of $h(f)$ simply means there is a high likelihood for $f$ to have appeared locally in the whole time span of the underlying signal [26]. If the 6MWT gait time series is comprised of $m$ gait cycles, the marginal spectra represents a set $\left\{h_{i}(f)\right\}_{i=1}^{m}$, consisting of one marginal spectrum per gait cycle. We transform the marginal spectrum into what we call the mean magnitude spectral density (MMSD), $\bar{h}(f)$. The MMSD is obtained by averaging the marginal spectra over $m$ giving the mean magnitude contribution from individual frequency components. Mathematically, MMSD can be written as

$$
\bar{h}(f)=\frac{\sum_{i=1}^{m} h_{i}(f)}{m}, f \leq f_{\max } .
$$

Frequencies that appear consistently in most of the gait cycles will have a higher

value of $\bar{h}(f)$. We compute MMSD and extract twelve spectral features for each of the three windows described above. These features, along with their descriptions, are given in Table 8.1. 
Table 8.1: Summary of the spectral features

\begin{tabular}{l|l}
\hline \hline Spectral feature & Description \\
\hline \hline$f_{m}$ & $\begin{array}{l}\text { The frequency that contributes the most to the spectrum, i.e., } \\
\text { the frequency at which the peak of the MMSD occurs }\end{array}$ \\
\hline Peak MMSD $\left(\bar{h}_{m}\right)^{1}$ & The maximum magnitude contribution from any frequency \\
\hline Band power $\left(P_{\text {band }}\right)^{1}$ & Average power \\
\hline Mean frequency $\left(f_{\text {mean }}\right)^{1}$ & $\begin{array}{l}\text { The sum of product of the MMSD and the frequency divided by } \\
\text { the sum of the MMSD [118] }\end{array}$ \\
\hline Median frequency $\left(f_{\text {median }}\right)^{1}$ & $\begin{array}{l}\text { The frequency at which the MMSD is divided into two regions } \\
\text { of equal magnitude }[118]\end{array}$ \\
\hline Occupied bandwidth $(\mathrm{OBW})$ & $99 \%$ occupied bandwidth \\
\hline Power bandwidth $\left(P_{B W}\right)^{1}$ & Half power bandwidth of the MMSD \\
\hline Skewness & Skewness of the spectrum \\
\hline Kurtosis & Kurtosis of the spectrum \\
\hline Total area under MMSD $\left(P_{M M S D}\right)^{1}$ & $\begin{array}{l}\text { The square-root of the total power contained in the MMSD } \\
0 \leq f \leq f_{\text {max }}\end{array}$ \\
\hline Area under main lobe of the spec- & $\begin{array}{l}\text { The magnitude contribution from low frequencies } \\
\text { trum }\left(P_{\text {main }}\right)^{1}\end{array}$ \\
\hline Area under MMSD tail $\left(P_{\text {tail }}\right)$ & $\begin{array}{l}\text { The magnitude contribution from high frequencies } \\
3 \leq f \leq 5 \mathrm{~Hz}\end{array}$ \\
\hline \hline
\end{tabular}

${ }^{1}$ Selected features

\subsection{Relationships of Clinical Data to Spectral Fea-}

\section{tures}

This section describes the physiological significance of spectral features and gait phases.

\subsubsection{Statistical Analysis}

Feature selection. We compute the Spearman correlation coefficients in order to identify the spectral features that have statistically significant correlations with the clinical sub-scores. The seven chosen spectral features are marked in Table 8.1 above. In addition, some of the clinical categories showed relationship to none of the spectral features, and are not included in the rest of the analysis. 
Regression models. Once spectral features and clinical categories are chosen, we generate linear regression models (LRMs) $\mathcal{M}_{X}^{Y}$, predicting response $Y$ from independent variables $X$, using model parameters described in Chapter 5. A total of 77 models were fitted for the whole gait cycle and its three windows with

- $X \in\{$ Clinical data discussed in Chapter 3$\}$

- $Y \in\{$ Spectral features from Table 8.1\}

The adjusted $R^{2}$ values from LRMs are given in Table 8.2. The results are discussed in the next section.

\subsubsection{Results and Discussions}

Spectral features. It is apparent from the table that spectral features show strong relationships with various types of clinical categories, although some of these relationships are weaker than others. Some of the spectral features measure similar types of impairment. The most populated and strongest relationships of clinical data are with the band power. The important thing to note is that it captures the functional status mainly in the first window. Bandwidth power is also an interesting feature as it is related to problems in doing daily activities in the second window and to cognitive/concentration impairment in the third window. Area under main lobe of the spectrum shows stronger relationships with balance and cognitive/concentration in the third window compared to other parts, and in fact, the entire gait cycle.

The mean and the median frequencies are not as informative as some of the other spectral features in the three windows but are statistically significant when using the entire gait cycle. The mean frequency is a stronger predictor of MFIS and fatigue-related disability while the median is more closely related to MSWS and motor disability. Overall, the mean frequency explains the variance in problems related 
Table 8.2: The \% of variance in the spectral features predicted by the clinical data

\begin{tabular}{|c|c|c|c|c|c|c|c|}
\hline Clinical data $^{1}$ & 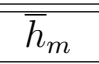 & 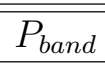 & $\overline{f_{\text {mean }}}$ & $\overline{f_{\text {median }}}$ & $\overline{P P_{B W}}$ & 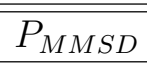 & 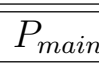 \\
\hline FSS I $^{2}$ & -4 & 29.6 & - & - & - & - & - \\
\hline $\mathrm{FSS} \mathrm{III}^{2}$ & - & - & - & - & - & - & 23.4 \\
\hline $\mathrm{FSS}^{* 3}$ & 36.8 & 40.2 & - & 30.5 & - & 33.9 & - \\
\hline EDSS I & - & 28.1 & - & - & - & - & - \\
\hline EDSS* & 44.0 & 47.3 & 26.6 & 36.7 & - & 41.7 & 28.1 \\
\hline Daily activities I & 30.7 & 51.8 & - & - & - & 41.8 & 41.2 \\
\hline Daily activities II $^{2}$ & - & 2167 & - & - & 25.2 & - & - \\
\hline Daily activities* & 45.2 & 55.3 & 39.3 & 48.0 & - & 47.7 & 42.2 \\
\hline MFIS III & - & - & - & - & - & - & 21.4 \\
\hline MFIS* & 32.0 & 36.1 & 31.4 & 22.1 & - & 33.2 & 20.4 \\
\hline MSWS I & - & 35.8 & - & - & - & 23.6 & 21.5 \\
\hline MSWS III & - & - & - & - & - & - & 26.7 \\
\hline MSWS* & 44.9 & 54.4 & 30.3 & 39.6 & - & 50.5 & 36.7 \\
\hline PDDS I & - & 33.6 & - & - & - & 21.3 & - \\
\hline PDDS* & 43.4 & 53.7 & 25.8 & 38.3 & - & 47.8 & 33.4 \\
\hline $\mathrm{PS}_{1} \mathrm{I}$ & - & 31.7 & - & - & - & 20.4 & - \\
\hline $\mathrm{PS}_{1} *$ & 45.5 & 57.9 & 31.8 & 44.8 & - & 49.1 & 35.7 \\
\hline Weakness I & - & 35.9 & - & - & - & 23.1 & 20.7 \\
\hline Weakness III & - & - & - & - & - & - & 22.8 \\
\hline Weakness* & 44.0 & 56.3 & 22.2 & 40.1 & - & 47.3 & 33.5 \\
\hline Physical fatigue I & - & 23.2 & - & - & - & - & - \\
\hline Physical fatigue III & - & - & - & - & - & - & 23.0 \\
\hline Physical fatigue* & 34.4 & 35.2 & - & 25.2 & - & 35.8 & 23.2 \\
\hline Balance I & - & 32.8 & - & - & - & 20.7 & - \\
\hline Balance III & - & 20.7 & - & - & - & 23.5 & 32.1 \\
\hline Balance* & 40.4 & 54.2 & 21.2 & 35.5 & - & 43.3 & 31.0 \\
\hline Cognitive and Concentration I & - & 33.4 & - & - & - & - & - \\
\hline Cognitive and Concentration III & - & - & - & - & 25.0 & 20.7 & 38.5 \\
\hline Cognitive and Concentration* & 44.2 & 50.0 & 35.0 & 24.8 & - & 39.7 & 31.9 \\
\hline
\end{tabular}

to cognitive/concentration, whereas the median frequency explains the variance in clinical data measuring weakness and balance disability. The proportion of total variation of balance and cognitive/concentration explained by the total area under MMSD is similar in the first and third window. In addition, this feature also shows 
a strong relationship with daily activities in the first window.

Importance of gait phases. Statistical features individually contribute to explain variance in different disability measures. To motivate the significance of eventspecific analysis, we generate LRMs $\mathcal{M}_{X}^{Y}$, predicting response $Y$ using independent variables $X$, with

- $X \in\{$ Spectral features from Table 8.1 $\}$

- $Y \in\{$ Clinical data discussed in Chapter 3$\}$

The adjusted $R^{2}$ values from LRMs are given in Table 8.3.

From previous studies and work presented in this dissertation, we already know that gait cycles capture physical and neurologic disability in MS, as is also validated from $R^{2}$ values given in the last column of Table 8.3. But the interesting finding is that most of the cognition and fatigue related information is present only in the third window. Thus, the TO to mid-swing phase of a gait cycle gives as much information, regarding the MS-induced fatigue and cognitive deterioration, as does the the state of an entire gait cycle

The goal of extracting phase-specific spectral features is to identify precise impact of MS symptoms on gait cycle components. This information could be used to develop disease-modifying therapies and exercises. The clinical relevance of spectral features to the three gait phases is given below:

1. Window I (Mid-swing to HS). This phase is a predictor of motor problems, captured using all MSWS components and its total score, tasks of daily living, and performance scale on mobility.

2. Window II (Foot-flat). It might not be intuitive, but findings suggest that the disturbed state of the gait between HS and TO (when the foot touches 
the ground and completely settles on it, before being carried forward into the next stride), indicates limitations in performing instrumental activities of daily living, namely, "getting beyond walking distance", "grocery shopping", and "doing laundry", in the order of the strongest to weakest prediction, as apparent from the Table 8.3.

3. Window III (TO to mid-swing). This is the acceleration phase, the one in which forward movement is being made in order to carry the body weight forward. This phase is a measure of overall disability status, including physical, functional, and most importantly, cognitive components. In fact, it is the only phase that explains the variance in clinical data quantifying impact of fatigue on persons with MS. The clinical categories include MFIS (Q 4, 6, 9-10, 13, 20 and total score), SF36 (Q4 d and g), all MSWS components and its total score, FSS components (cerebellar, pyramidal, and bladder), and EDSS.

\subsection{Summary}

Gait cycles capture physical and neurologic disability in MS, as is also validated from previous studies and results presented in this dissertation. Based on eventspecific study, we found that most of the information regarding disability in persons with MS is contained in the TO to mid-swing and mid-swing to HS phases. The mid-swing to HS phase is specific to motor function. The TO to mid-swing phase is indicative of overall health status, including functional, physical, and cognitive components. Most importantly, it is the only phase that measures the impacts of motor-fatigue on persons with MS. So analyzing TO to mid-swing phase of a gait cycle to measure MS-induced fatigue and cognitive impairment is as good as analyzing

the state of an entire gait cycle. It is interesting to know that the the foot-flat 
phase, the transition between HS and TO, showed statistically strong and significant relationships to patient-reported outcomes regarding the limitations in performing activities of daily living.

We conclude that the event-based analysis could help researchers and clinicians identify precise impact of MS symptoms on gait cycle components, and therefore, on neurological, functional, and physical functions. We believe that this information is specific and could be used to better understand the personal needs of an individual and develop customized disease-modifying therapies and exercises. 
Table 8.3: The adjusted $R^{2}$ showing the proportion of variance in clinical sub-scores as explained by the spectral features

\begin{tabular}{|c|c|c|c|c|}
\hline Clinical data $^{1}$ & Window I & Window II & Window III & ${\text { Complete gait } \text { cycle }^{2}}$ \\
\hline SF36 Q4d & 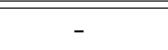 & 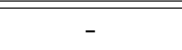 & $\overline{23.3}$ & 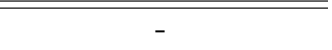 \\
\hline SF36 Q4g & - & - & 22.0 & - \\
\hline MFIS Q13 & - & - & 22.1 & - \\
\hline MFIS total & - & - & 20.5 & 20.3 \\
\hline FSS cerebellar & - & - & 21.5 & 20.8 \\
\hline Performance scale spasticity & - & - & - & 20.9 \\
\hline MFIS Q21 & - & - & - & 22.2 \\
\hline Performance scale fatigue & - & - & - & 22.3 \\
\hline MFIS Q4 & - & - & 23.6 & 24.3 \\
\hline MFIS Q10 & - & - & 25.6 & 26.0 \\
\hline MFIS Q17 & - & - & - & 26.1 \\
\hline Hand function & - & - & - & 26.4 \\
\hline FSS pyramidal & - & - & 28.1 & 26.6 \\
\hline MFIS Q20 & - & - & 26.8 & 29.1 \\
\hline LLE Ashworth & - & - & - & 30.5 \\
\hline MFIS Q6 & - & - & 29.9 & 30.8 \\
\hline SF36 Q4j & - & - & - & 32.8 \\
\hline MFIS Q9 & - & - & 27.3 & 33.7 \\
\hline MSWS Q2 & 20.9 & - & - & 37.1 \\
\hline FSS bladder & - & - & 22.8 & 37.1 \\
\hline RLE Ashworth & - & - & - & 38.6 \\
\hline MSWS Q4 & 23.1 & - & 21.9 & 39.6 \\
\hline MSWS Q12 & 24.1 & - & 42.4 & 39.7 \\
\hline MSWS Q6 & 23.2 & - & 39.1 & 40.0 \\
\hline Preparing meals & 41.1 & - & - & 42.0 \\
\hline Getting beyond walking distance & 44.0 & 33.3 & - & 43.2 \\
\hline Doing housework & 37.9 & - & - & 43.8 \\
\hline MSWS Q11 & 28.0 & - & 38.2 & 45.8 \\
\hline MSWS Q5 & 25.7 & - & 39.4 & 46.0 \\
\hline MSWS Q7 & 25.1 & - & 41.2 & 46.5 \\
\hline Grocery shopping & 49.7 & 33.0 & - & 46.6 \\
\hline MSWS Q10 & 29.2 & - & 31.2 & 47.5 \\
\hline MSWS Q1 & 31.3 & - & 32.7 & 49.0 \\
\hline Doing laundry & 48.4 & 22.2 & - & 49.6 \\
\hline MSWS Q3 & 29.7 & - & 40.9 & 50.7 \\
\hline MSWS total & 31.5 & - & 44.0 & 51.2 \\
\hline MSWS Q8 & 35.9 & - & 42.7 & 52.5 \\
\hline EDSS & 31.6 & - & 40.3 & 54.7 \\
\hline IADL score & 53.7 & - & - & 55.3 \\
\hline PDDS & 36.8 & - & 28.6 & 59.5 \\
\hline MSWS Q9 & 39.1 & - & 40.9 & 60.5 \\
\hline Performance scale mobility & 37.2 & - & 38.0 & 63.4 \\
\hline
\end{tabular}

${ }^{1}$ From Table 3.1

${ }^{2}$ Rows are sorted in order of increasing adjusted $R^{2}$ values for complete gait cycle 


\section{Chapter 9}

\section{SUMMARY AND CONCLUSION}

Walking, an act of repeating cyclic gestures or gait cycles, is known to be a complex cognitive task. To carry out this task, the nervous system and musculoskeletal system must function and coordinate properly to generate a sequence of events to complete the process of walking. Thus, the quality of walking is degraded if neurology is disturbed or if the communication between the nervous system and the rest of the body is interrupted. Gait dynamics carry substantial information about human heath as do other vital signals such as heartbeat and pulse. Gait analysis has become a handy tool in medicine and general fitness and health maintenance.

Our research goals were characterizing MS-affected gait using inertial data and mining their physiological significance. Existing literature targets finding gait variables that improve separability performance to distinguish between controls and MS subjects with or without reference to MS disability levels, assigned using a subjective, ordinal scale. Often, the relationships between inertial gait variables and clinical data are not explored. We developed gait markers taking into account a few of the current unmet needs. Our inertial markers verify three hypotheses:

1. There is an inverse relationship between stride time stability and worsening of 
symptoms (results in Chapter 6).

2. The peaks of kernel density estimates verify that pathologic gait in MS is restricted and less variable in comparison to a healthy gait (results in Chapter 7).

3. The disturbances in various gait phases predict distinct types of MS-induced disabilities (results in Chapter 8).

Our results also suggest that in the early stages of the disease, when motor functions are not significantly affected, the walk appears normal, i.e., gait variables of persons with MS may be statistically similar to those of control subjects. Thus, mild MS subjects are difficult to distinguish since their features overlap with control subjects.

To make our test measures accessible for the doctor, we used a data mining perspective to discover the relationships of gait variables with clinical data. Regression results show that subjects with similar self- or physician-rated clinical scores might not belong to the same group (or the other way around), either due the fact that subjective ratings are imprecise, insensitive to subtle changes in gait, or because of the impact of type and severity of symptoms on specific functions.

The components of MSWS, MFIS, and FSS can be grouped into four groups, intended to gauge the crucial types of disabilities caused by MS, namely, weakness, physical fatigue, balance, and cognitive/concentration. To investigate the physiological significance of our feature space, we perform a regression analysis to determine the proportion of variation in inertial features as explained by the domains of MS-induced disability, in terms of the adjusted $R^{2}$. The results are summarized in Table 9.1. The feature space shows significant correlations with various types of MS-induced disability, although some of the relationships are stronger than others. Table 9.2 show the $\%$ contributions of MS disability groups to the inertial measures. 
Table 9.1: Summary of the results from Chapters 6 to 8

\begin{tabular}{l|c|c|c|c}
\hline \hline Inertial measures & Weakness & $\begin{array}{c}\text { Physical } \\
\text { fatigue }\end{array}$ & Balance & $\begin{array}{c}\text { Cognitive/ } \\
\text { concentration }\end{array}$ \\
\hline 6MWT speed & $77.8^{1}$ & 63.6 & 76.7 & 68.9 \\
\hline Stride time SD, $\sigma_{s t}$ gradient, & 41.8 & 16.6 & 38.9 & 51.5 \\
\hline $\begin{array}{l}\text { Stride time } \\
\Delta_{6 M W T}\end{array}$ & 53.9 & 30.8 & 60.0 & 38.4 \\
\hline KDE peak, $\rho$ & 44.0 & 34.4 & 40.4 & 44.2 \\
\hline MMSD peak, $\bar{h}_{m}$ & 56.3 & 35.2 & 54.2 & 50.0 \\
\hline MMSD bandpower, $P_{\text {band }}$ & 22.2 & 18.6 & 21.2 & 35.1 \\
\hline Mean frequency, $f_{\text {mean }}$ & 40.1 & 25.2 & 35.5 & 24.8 \\
\hline Median frequency, $f_{\text {median }}$ & 47.3 & 35.9 & 43.3 & 39.7 \\
\hline $\begin{array}{l}\text { Total area under MMSD, } \\
P_{M M S D}\end{array}$ & 33.5 & 23.2 & 31.0 & 31.9 \\
\hline $\begin{array}{l}\text { Total area under main lobe } \\
\text { of MMSD, } P_{\text {main }}\end{array}$ & & & \\
\hline \hline
\end{tabular}

1 The adjusted $R^{2}$ values, the proportion of variation in different inertial measures as explained by the distinct MS disabilities, are shown.

\section{Table 9.2: The \% contributions of various aspects of disability in MS to the inertial measures}

\begin{tabular}{l|c|c|c|c}
\hline \hline Inertial measures & Weakness & $\begin{array}{c}\text { Physical } \\
\text { fatigue }\end{array}$ & Balance & $\begin{array}{c}\text { Cognitive/ } \\
\text { concentration }\end{array}$ \\
\hline 6MWT speed & 27.1 & 22.2 & 26.7 & 24.0 \\
\hline Stride time SD, $\sigma_{s t}$ gradient, & 28.5 & 15.2 & 28.7 & 27.2 \\
\hline $\begin{array}{l}\text { Stride time } \\
\Delta_{6 M W T}\end{array}$ & 29.4 & 11.2 & 26.2 & 34.6 \\
\hline KDE peak, $\rho$ & 27.0 & 21.1 & 24.8 & 27.1 \\
\hline MMSD peak, $\bar{h}_{m}$ & 28.8 & 18.0 & 27.7 & 25.5 \\
\hline MMSD bandpower, $P_{\text {band }}$ & 22.9 & 19.1 & 21.8 & 36.1 \\
\hline Mean frequency, $f_{\text {mean }}$ & 31.9 & 20.1 & 28.2 & 19.7 \\
\hline Median frequency, $f_{\text {median }}$ & 28.4 & 21.6 & 26.1 & 23.9 \\
\hline $\begin{array}{l}\text { Total area under MMSD, } \\
P_{M M S D}\end{array}$ & 28.0 & 19.4 & 25.9 & 26.7 \\
\hline $\begin{array}{l}\text { Total area under main lobe } \\
\text { of MMSD, } P_{\text {main }}\end{array}$ & & & \\
\hline \hline
\end{tabular}

Walking speed is the core of clinical assessments as a best measure of neurologic and motor disability. Our results show that walking speed has the strongest relation- 
ships to all the aspects of MS disability. The association of walking speed with various neurological impairment affecting walking is intuitive, and has also been validated by many past studies. Thus, walking speed is an indicator of overall health and mobility status. Other inertial measures are more specialized but are not intended to serve as substitutes for walking speed, but rather to supplement it.

Without proper management of symptoms, the disease progresses, and impairment worsens, and the four types of impairment no longer remain disjoint. For example, cognitive/concentration problems may lead to balance problems. Hence, although capturing distinct types of disabilities, the inertial measures show correlations to all the disability categories, with some relations being stronger than the others.

The MMSD peak has equally strong and significant relationships with the weakness and cognitive/concentration. Both standard deviation stride time and its gradient have the weakest relationship with physical fatigue, the corresponding adjusted $R^{2}$ value for the gradient is, however, surprisingly small. Interestingly, the gradient of the stride time is the most sensitive to impairment in cognition or concentration than other types of disabilities, with a difference of $\approx 10 \%$ more than the other categories. Thus, a high gradient value is more of a result due to problems in cognition or concentration that those in weakness or balance. Peak of the kernel density estimate is the best indicator of weakness and balance issues, and thus can be used to predict fall risk. The band power and stride time standard deviation give similar information regarding weakness, balance, or cognitive/concentration problems, but the band power also has a relatively strong relationships with the physical fatigue. The mean frequency is particularly is the most sensitive to cognitive/concentration, and thus can be used as an effective objective measure to quantify the impaired cognition or concentration. These correlations validate inertial features as useful clinical anchors. For visualization, values given in the Table 9.1 and Table 9.2 are plotted in Fig. 9.1 
and Fig. 9.2, respectively.

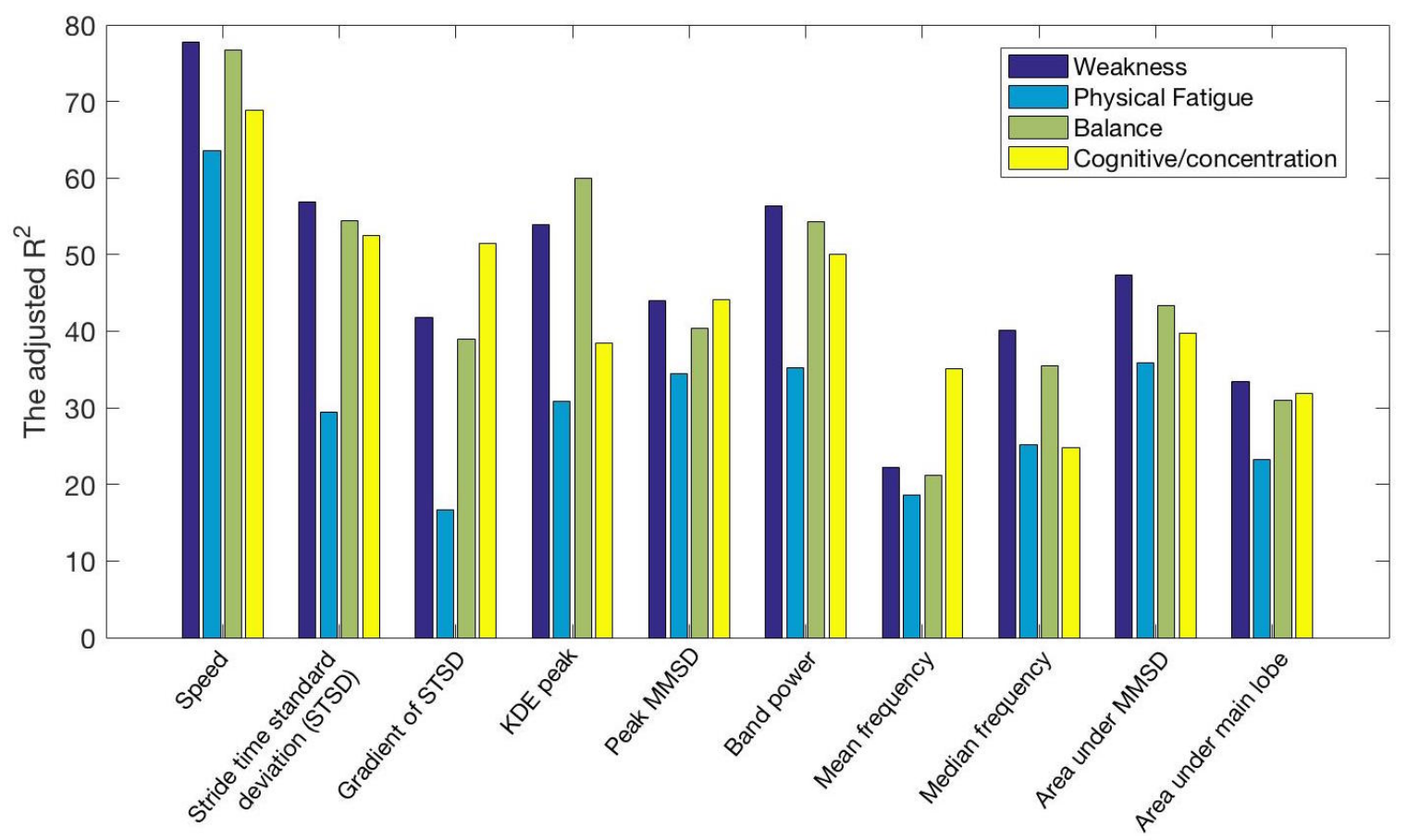

Fig. 9.1: The variance in gait features explained by MS disability aspects

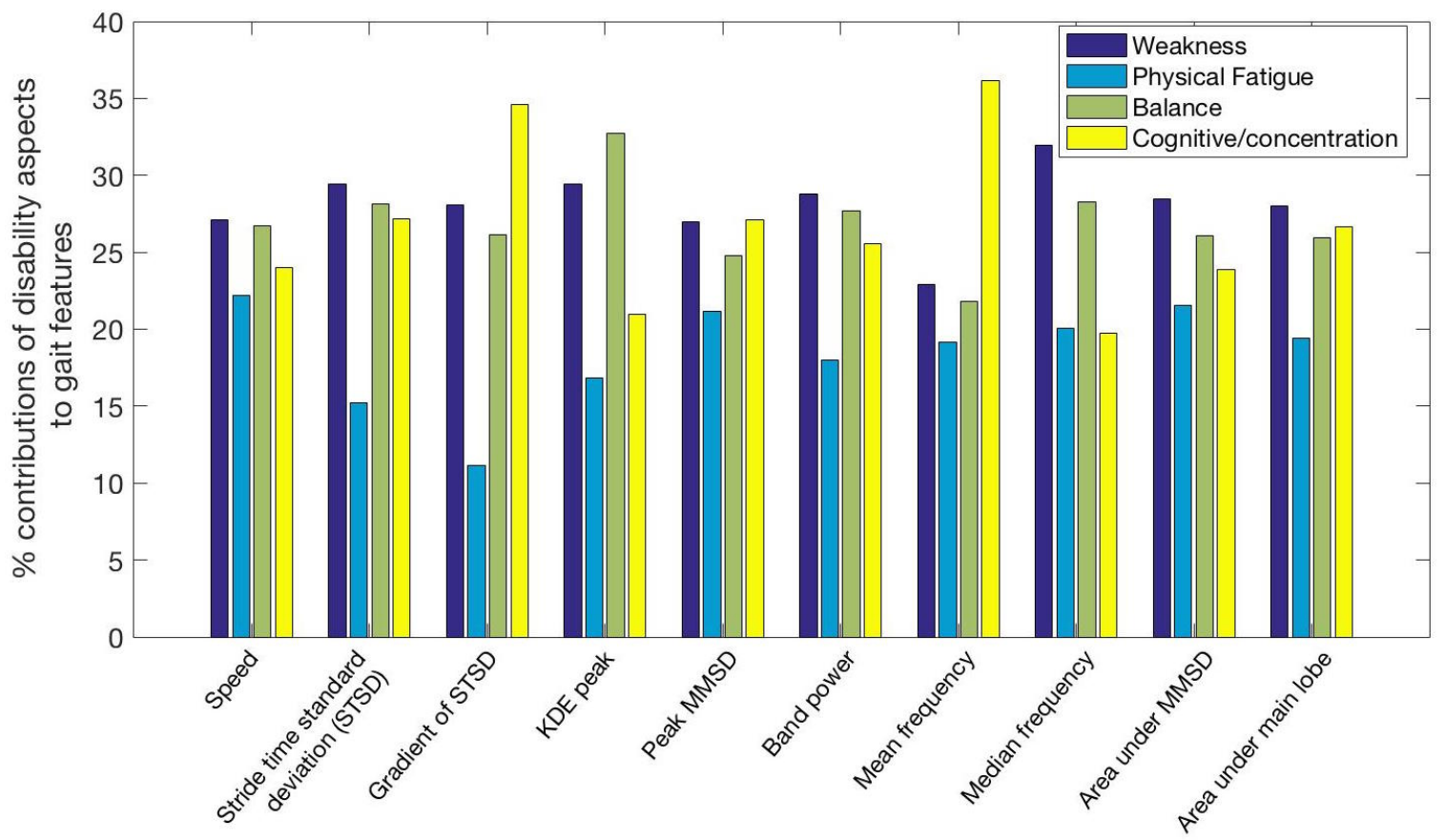

Fig. 9.2: The \% contributions of MS disabilities to the inertial measures 
One of our motivations for looking at gait data is to determine whether there are features which vary across the MS population but do not correlate strongly with any clinically obtained data. Although the KDE peak shows significant relationships with the clinical data specific to weakness and balance deterioration, the gait amplitude at which the KDE peak occurs did not show any relationship with the clinical data. This can be investigated further as it might be an indication of missing information from the clinical assessments.

Numerous questionnaires are developed to assess the physical and cognitive disability in MS, e.g., the gait imparment in MS is captured using MSWS as well as a performance scale on mobility. The inertial features show strong relationship with both metrics. As future work, a study can be conducted to determine which scale is better based on its relationships with various gait parameters.

EDSS based categorizations of subjects into disease severity groups are not sharp, especially for low disability groups $(0 \leq \mathrm{EDSS} \leq 4)$. The classifications of MS subjects using the PROs are, in fact, more telling, and also track with the inertial measures. EDSS is a composite score, with some of the scores not directly related to walking quality, such as vision. In the future, these correlations between the EDSS, PROs, and inertial measures can be carefully studied to refine EDSS. The best combinations of the inertial features should be identified to help assess and predict disability. Moreover, some of the inertial features have similar physiological meaning, e.g., the KDE peak and the median frequency of MMSD contribute equally to balance and weakness. A way to determine the best-suited measure depending on the clinical or research goal is needed. Last but not the least, the adaptability of these measures to other neurological disorders should be tested.

Our work is intended towards more comprehensive exploration of inertial features and discovering their physical significance to add clinical utility to them. Our new, 
objective anchors can be used in addition to traditional speed-based assessments and/or other inertial features available in literature, to improve physician's capacity to monitor the disease and its progression, evaluate the effectiveness of the treatments, follow recovery and rehabilitation, tailor subjective assessments based on individual needs, and guide out-of-clinic, self-management of symptoms by the individuals. Although our goals were motivated by the study a specific neurological disorder, the developed test measures could be adapted for gait assessments and monitoring in other neurological disorders affecting gait or balance, fall risk prediction, and general health and wellness applications. We believe that, through this research, we have augmented the existing information and on-going research to support medical and research community, and done a small part in improving the quality of life. 


\section{Appendix}

\section{Subjective Questionnaires}

Clinical data comprising of patient-reported and physician-reported clinical subscores are obtained using standard forms. Following questionnaires are attached in the appendix:

1. Functional systems scores (FSS) [7]

2. Expanded disability status scale (EDSS) [7]

3. Multiple sclerosis walking scale (MSWS) [48, 95]

4. Modified fatigue impact scale (MFIS) [10]

5. Health status questionnaire (SF36) [10, 96]

6. Patient-determined disease steps (PDDS) [98]

7. Performance scales for mobility, hand, vision, fatigue, cognitive, bladder/bowel, sensory, spasticity, pain, depression, and tremor [92]

8. Modified Ashworth [94] 


\section{Kurtzke Functional Systems Scores (FSS)}

\section{Pyramidal Functions}

0 - Normal

1 - Abnormal signs without disability

2 - Minimal disability

3 - Mild to moderate paraparesis or hemiparesis (detectable weakness but most function sustained for short periods, fatigue a problem); severe monoparesis (almost no function)

4 - Marked paraparesis or hemiparesis (function is difficult), moderate quadriparesis (function is decreased but can be sustained for short periods); or monoplegia

5 - Paraplegia, hemiplegia, or marked quadriparesis

6 - Quadriplegia

9 - (Unknown)

\section{Cerebellar Functions}

0 - Normal

1 - Abnormal signs without disability

2 - Mild ataxia (tremor or clumsy movements easily seen, minor interference with function)

3 - Moderate truncal or limb ataxia (tremor or clumsy movements interfere with function in all shpheres)

4 - Severe ataxia in all limbs (most function is very difficult)

5 - Unable to perform coordinated movements due to ataxia

9 - (Unknown)

Record \#1 in small box when weakness (grade 3 or worse on pyramidal) interferes with testing.

\section{Brainstem Functions}

0 - Normal

1 - Signs only

2 - Moderate nystagmus or other mild disability

3 - Severe nystagmus, marked extraocular weakness, or moderate disability of other cranial nerves

4 - Marked dysarthria or other marked disability

5 - Inability to swallow or speak

9 - (Unknown) 


\section{Sensory Function}

0 - Normal

1 - Vibration or figure-writing decrease only in one or two limbs

2 - Mild decrease in touch or pain or position sense, and/or moderate decrease in vibration in one or two limbs; or vibratory (c/s figure writing) decrease alone in three or four limbs

3 - Moderate decrease in touch or pain or position sense, and/or essentially lost vibration in one or two limbs; or mild decrease in touch or pain and/or moderate decrease in all proprioceptive tests in three or four limbs

4 - Marked decrease in touch or pain or loss of proprioception, alone or combined, in one or two limbs; or moderate decrease in touch or pain and/or severe proprioceptive decrease in more than two limbs

5 - Loss (essentially) of sensation in one or two limbs; or moderate decrease in touch or pain and/or loss of proprioception for most of the body below the head

6 - Sensation essentially lost below the head

9 - (Unknown)

\section{Bowel and Bladder Function}

(Rate on the basis of the worse function, either bowel or bladder)

0 - Normal

1 - Mild urinary hesitance, urgency, or retention

2 - Moderate hesitance, urgency, retention of bowel or bladder, or rare urinary incontinence (intermittent self-catheterization, manual compression to evacuate bladder, or finger evacuation of stool)

3 - Frequent urinary incontinence

4 - In need of almost constant catheterization (and constant use of measures to evacuate stool)

5 - Loss of bladder function

6 - Loss of bowel and bladder function

9 - (Unknown) 


\section{Visual Function}

0 - Normal

1 - Scotoma with visual acuity (corrected) better than 20/30

2 - Worse eye with scotoma with maximal visual acuity (corrected) of 20/30-20/59

3 - Worse eye with large scotoma, or moderate decrease in fields, but with maximal visual acuity (corrected) of 20/60-20/99

4 - Worse eye with marked decrease of fields and maximal visual acuity (corrected) of 20/100-20/200; grade 3 plus maximal acuity of better eye of 20/60 or less

5 - Worse eye with maximal visual acuity (corrected) less than 20/200; grade 4 plus maximal acuity of better eye of $20 / 60$ or less

6 - Grade 5 plus maximal visual acuity of better eye of 20/60 or less

9 - (Unknown)

Record \#1 in small box for presence of temporal pallor

\section{Cerebral (or Mental) Functions}

0 - Normal

1 - Mood alteration only (does not affect EDSS score)

2 - Mild decrease in mentation

3 - Moderate decrease in mentation

4 - Marked decrease in mentation (chronic brain syndrome - moderate)

5 - Dementia or chronic brain syndrome - severe or incompetent

9 - (Unknown)

Sources: Kurtzke JF. Rating neurologic impairment in multiple sclerosis: an expanded disability status scale (EDSS). Neurology. 1983 Nov;33(11):1444-52.

Haber A, LaRocca NG. eds. Minimal Record of Disability for multiple sclerosis. New York: National Multiple Sclerosis Society; 1985. 


\section{Kurtzke Expanded Disability Status Scale (EDSS)}

0.0 - Normal neurological exam (all grade 0 in all Functional System (FS) scores*).

1.0 - No disability, minimal signs in one $\mathrm{FS}^{*}$ (i.e., grade 1 ).

1.5 - No disability, minimal signs in more than one FS* (more than 1 FS grade 1 ).

2.0 - Minimal disability in one FS (one FS grade 2, others 0 or 1 ).

2.5 - Minimal disability in two FS (two FS grade 2, others 0 or 1 ).

3.0 - Moderate disability in one FS (one FS grade 3 , others 0 or 1 ) or mild disability in three or four FS (three or four FS grade 2, others 0 or 1 ) though fully ambulatory.

3.5 - Fully ambulatory but with moderate disability in one FS (one grade 3 ) and one or two FS grade 2; or two FS grade 3 (others 0 or 1 ) or five grade 2 (others 0 or 1 ).

4.0 - Fully ambulatory without aid, self-sufficient, up and about some 12 hours a day despite relatively severe disability consisting of one FS grade 4 (others 0 or 1), or combination of lesser grades exceeding limits of previous steps; able to walk without aid or rest some 500 meters.

4.5 - Fully ambulatory without aid, up and about much of the day, able to work a full day, may otherwise have some limitation of full activity or require minimal assistance; characterized by relatively severe disability usually consisting of one FS grade 4 (others or 1) or combinations of lesser grades exceeding limits of previous steps; able to walk without aid or rest some 300 meters.

5.0 - Ambulatory without aid or rest for about 200 meters; disability severe enough to impair full daily activities (e.g., to work a full day without special provisions); (Usual FS equivalents are one grade 5 alone, others 0 or 1 ; or combinations of lesser grades usually exceeding specifications for step 4.0).

5.5 - Ambulatory without aid for about 100 meters; disability severe enough to preclude full daily activities; (Usual FS equivalents are one grade 5 alone, others 0 or 1; or combination of lesser grades usually exceeding those for step 4.0).

6.0 - Intermittent or unilateral constant assistance (cane, crutch, brace) required to walk about 100 meters with or without resting; (Usual FS equivalents are combinations with more than two FS grade $3+$ ). 
6.5 - Constant bilateral assistance (canes, crutches, braces) required to walk about 20 meters without resting; (Usual FS equivalents are combinations with more than two FS grade $3+)$.

7.0 - Unable to walk beyond approximately 5 meters even with aid, essentially restricted to wheelchair; wheels self in standard wheelchair and transfers alone; up and about in wheelchair some 12 hours a day; (Usual FS equivalents are combinations with more than one FS grade 4+; very rarely pyramidal grade 5 alone).

7.5 - Unable to take more than a few steps; restricted to wheelchair; may need aid in transfer; wheels self but cannot carry on in standard wheelchair a full day; May require motorized wheelchair; (Usual FS equivalents are combinations with more than one FS grade $4+$ ).

8.0 - Essentially restricted to bed or chair or perambulated in wheelchair, but may be out of bed itself much of the day; retains many self-care functions; generally has effective use of arms; (Usual FS equivalents are combinations, generally grade 4+ in several systems).

8.5 - Essentially restricted to bed much of day; has some effective use of arm(s); retains some self-care functions; (Usual FS equivalents are combinations, generally 4+ in several systems).

9.0 - Helpless bed patient; can communicate and eat; (Usual FS equivalents are combinations, mostly grade $4+$ ).

9.5 - Totally helpless bed patient; unable to communicate effectively or eat/swallow; (Usual FS equivalents are combinations, almost all grade 4+).

10.0 - Death due to MS.

*Excludes cerebral function grade 1.

Note 1: EDSS steps 1.0 to 4.5 refer to patients who are fully ambulatory and the precise step number is defined by the Functional System score(s). EDSS steps 5.0 to 9.5 are defined by the impairment to ambulation and usual equivalents in Functional Systems scores are provided.

Note 2: EDSS should not change by 1.0 step unless there is a change in the same direction of at least one step in at least one FS.

Sources: Kurtzke JF. Rating neurologic impairment in multiple sclerosis: an expanded disability status scale (EDSS). Neurology. 1983 Nov;33(11):1444-52.

Haber A, LaRocca NG. eds. Minimal Record of Disability for multiple sclerosis. New York: National Multiple Sclerosis Society; 1985. 
Twelve Item MS Walking Scale (Mswis-12) Record form

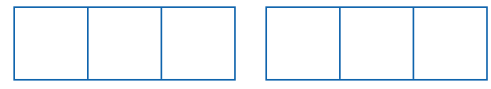

Subject ID Number

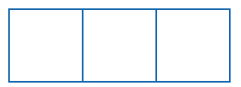

Subject Initials
Date
Questionnaire
Completed

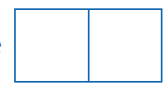

Day

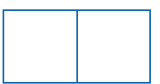

Month

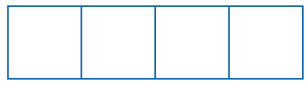

Year

If you cannot walk at all, please tick this box

\begin{tabular}{|c|c|c|c|c|c|}
\hline $\begin{array}{l}\text { In the past two weeks, } \\
\text { how much has your MS... }\end{array}$ & Not at all & A little & Moderately & $\begin{array}{l}\text { Quite } \\
\text { a lot }\end{array}$ & Extremely \\
\hline 1. Limited your ability to walk? & 1 & 2 & 3 & 4 & 5 \\
\hline 2. Limited your ability to run? & 1 & 2 & 3 & 4 & 5 \\
\hline 3. Limited your ability to climb up and down stairs? & 1 & 2 & 3 & 4 & 5 \\
\hline 4. Made standing when doing things more difficult? & 1 & 2 & 3 & 4 & 5 \\
\hline 5. Limited your balance when standing or walking? & 1 & 2 & 3 & 4 & 5 \\
\hline 6. Limited how far you are able to walk? & 1 & 2 & 3 & 4 & 5 \\
\hline 7. Increased the effort needed for you to walk? & 1 & 2 & 3 & 4 & 5 \\
\hline $\begin{array}{l}\text { 8. Made it necessary for you to use support when } \\
\text { walking indoors (eg holding on to furniture, } \\
\text { using a stick, etc.)? }\end{array}$ & 1 & 2 & 3 & 4 & 5 \\
\hline $\begin{array}{l}\text { 9. Made it necessary for you to use support when } \\
\text { walking outdoors (eg using a stick, a frame, etc.)? }\end{array}$ & 1 & 2 & 3 & 4 & 5 \\
\hline 10. Slowed down your walking? & 1 & 2 & 3 & 4 & 5 \\
\hline 11. Affected how smoothly you walk? & 1 & 2 & 3 & 4 & 5 \\
\hline 12. Made you concentrate on your walking? & 1 & 2 & 3 & 4 & 5 \\
\hline
\end{tabular}

From the numbers you circle against these questions, your healthcare professional can calculate your MSWS-12 score. This is done by adding the numbers you have circled, giving a total out of 60, and then transforming this to a scale with a range from 0 to 100. Higher scores indicate a greater impact on walking than lower scores.

\section{To be completed by the healthcare professional}

Total score out of 60

Percentage $\%$ 
Patient's Name:

ID\#:
Date:<smiles>CC(C)(C)C(C)(C)C</smiles>

$\begin{array}{lllll}\text { Test\#: } & 1 & 2 & 3 & 4\end{array}$

\section{MODIFIED FATIGUE IMPACT SCALE (MFIS)}

Following is a list of statements that describe how fatigue may affect a person. Fatigue is a feeling of physical tiredness and lack of energy that many people experience from time to time. In medical conditions like MS, feelings of fatigue can occur more often and have a greater impact than usual. Please read each statement carefully, and then circle the one number that best indicates how often fatigue has affected you in this way during the past 4 weeks. (If you need help in marking your responses, tell the interviewer the number of the best response.) Please answer every question. If you are not sure which answer to select, please choose the one answer that comes closest to describing you. The interviewer can explain any words or phrases that you do not understand.

Because of my fatigue

during the past 4 weeks....

\section{Almost \\ $\underline{\text { Never Rarely Sometimes Often always }}$}

1. I have been less alert. $\mathbf{0} 1$

2 $3 \quad 4$

2. I have had difficulty paying attention for long periods of time.

0 1 2 3 4

3. I have been unable to think clearly.

0 1
2 3 4

4. I have been clumsy and uncoordinated. 0 1

12
3 4

5. I have been forgetful. $\mathbf{0}$ 1 2 3 4

6. I have had to pace myself in my physical activities.

0

7. I have been less motivated to do anything that requires physical effort. 0 1 2 3 4 
Because of my fatigue

during the past 4 weeks....

8. I have been less motivated

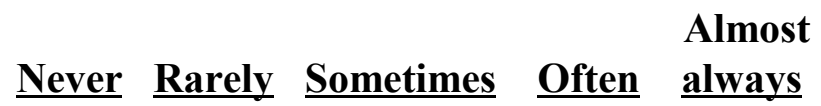
to participate in social activities.

0 1

2

3

4

9. I have been limited in my ability to do things away from home.

0 1 2 3 4

10. I have had trouble maintaining physical effort for long periods.

0 1
2 3 4

11. I have had difficulty making decisions.

0 1

2 3 4

12. I have been less motivated to do anything that requires thinking. 0 1 2 3 4

13. my muscles have felt weak. 0 1 2 3 4

14. I have been physically uncomfortable.

$\mathbf{0}$

1

2

3

4

15. I have had trouble finishing tasks that require thinking.

0

1

2

3

4

16. I have had difficulty

organizing my thoughts when doing things at home or at work.

0 1

2

3

4

17. I have been less able to complete tasks that require physical effort.

$\mathbf{0}$

1

2

3

4

18. my thinking has been slowed down.

0 1

12

3

4

19. I have had trouble concentrating. $\begin{array}{lll}\mathbf{0} & 1 & 2\end{array}$ 3 4 
Because of my fatigue

during the past 4 weeks....
20. I have limited my physical activities.

21. I have needed to rest more often or for longer periods.

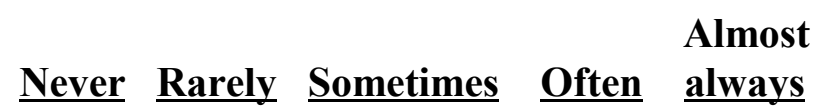

$\begin{array}{lll}0 & 1 & 2\end{array}$

3

4

4 
Patient's Name:

ID\#:
Date: $\overline{\text { month day year }}$

$\begin{array}{lllll}\text { Test\#: } & 1 & \mathbf{2} & \mathbf{3} & \mathbf{4}\end{array}$

\section{HEALTH STATUS QUESTIONNAIRE (SF-36)}

\section{INSTRUCTIONS}

This survey asks for your views about your health and daily activities. If you are marking your own answers, please circle the appropriate responses $(0,1,2, \ldots)$. If you need help in marking your responses, tell the interviewer the number of the best response (or what to fill in). Please answer every question. If you are not sure which answer to select, please choose the one answer that comes closest to describing you. The interviewer can explain any words or phrases that you do not understand.

1. In general, would you say your health is:

\begin{tabular}{ccccc} 
Excellent & Very Good & Good & Fair & Poor \\
\hline 1 & 2 & 3 & 4 & 5
\end{tabular}

2. For each statement please circle the one number that indicates how true or false that statement is for you.

$\begin{array}{ccccc}\text { Definitely } & \text { Mostly } & \text { Not } & \text { Mostly } & \text { Definitely } \\ \text { True } & \underline{\text { True }} & \underline{\text { Sure }} & \underline{\text { False }} & \underline{\text { False }}\end{array}$

a) I seem to get sick a little easier than other people. 123

$\begin{array}{lll}3 & 4 & 5\end{array}$

b) I am as healthy as anybody I know. 1 2 3 4 5

c) I expect my health to get worse. $12 \quad 3$ $3 \quad 4$ 5

d) My health is excellent. 12 3 45

3. Compared to one year ago, how would you rate your health in general now?

\begin{tabular}{cccccc}
$\begin{array}{l}\text { Much } \\
\text { Better }\end{array}$ & $\begin{array}{c}\text { Somewhat } \\
\text { Better }\end{array}$ & Same & \multicolumn{2}{c}{$\begin{array}{c}\text { Somewhat } \\
\text { Worse }\end{array}$} & $\begin{array}{l}\text { Much } \\
\text { Worse }\end{array}$ \\
\hline 1 & 2 & 3 & & 4 & 5
\end{tabular}


4. Now, think about the activities you might do on a typical day. Does your health limit you in these activities? If so, how much? Please circle 1, 2 or 3 for each item to indicate how much your health limits you.

$\begin{array}{ccc}\text { Yes, } & \text { Yes, } & \text { No, Not } \\ \text { Limited } & \text { Limited } & \text { Limited } \\ \underline{\text { A Lot }} & \underline{\text { A Little }} & \underline{\text { At All }}\end{array}$

a) Vigorous activities, such as running, lifting heavy objects, participating in strenuous sports

12
3

b) Moderate activities, such as moving a table, pushing a vacuum cleaner or bowling, or playing golf

$\begin{array}{lll}1 & 2 & 3\end{array}$

c) Lifting or carrying groceries 1 2 3

d) Climbing several flights of stairs 1 2 3

e) Climbing one flight of stairs 1 2 3 f) Bending, kneeling, or stooping 12 3

g) Walking more than a mile 1 2 3

h) Walking several blocks 1 2 3

i) Walking one block 1 2 3

5. During the past 4 weeks, have you had any of the following problems with your work or other regular daily activities as a result of your physical health? Please circle "1" (Yes) or "2" (No) for each item.

$\underline{\text { YES }} \quad \underline{\text { NO }}$

a) Cut down on the amount of time you spent on work or other activities 
During the past 4 weeks, have you had any of the following problems with your work or other regular daily activities as a result of your physical health? Please circle "1" (Yes) or "2" (No) for each item.

$\underline{\text { YES }} \quad \underline{\text { NO }}$

c) Were limited in the kind of work or other activities 12

d) Had difficulty performing the work or other activities 12 (for example, it took extra effort)

6. How much bodily pain have you had during the past 4 weeks?

\begin{tabular}{cccccc} 
None & Very mild & Mild & Moderate & Severe & Very severe \\
\hline 1 & 2 & 3 & 4 & 5 & 6
\end{tabular}

7. During the past 4 weeks, how much did pain interfere with your normal work (including work both outside the home and housework)?

Not at all

1
A little bit

2
Moderately

3
Quite a bit

4 Extremely 5

8. During the past 4 weeks, have you had the following problems with your work or other regular daily activities as a result of any emotional problems (such as feeling depressed or anxious)? Please circle "1" (Yes) or "2" (No) for each item.

\section{$\underline{\text { YES }} \quad \underline{\text { NO }}$}

a) Cut down on the amount of time you spent on work or other activities

1 2

b) Accomplished less than you would like 1 2

c) Did do work or other activities less carefully than usual 1 2

9. During the past 4 weeks, to what extent have your physical health or emotional problems interfered with your normal social activities with family, friends, neighbors, or groups?

\begin{tabular}{ccccc} 
Not at all & Slightly & Moderately & Quite a bit & Extremely \\
\hline 1 & 2 & 3 & 4 & 5
\end{tabular}


10. The next set of questions is about how you feel and how things have been with you during the past 4 weeks. For each question, please circle the one number for the answer that comes closest to the way you have been feeling.

How much of the time

during the past 4 weeks...

$\begin{array}{cccccc}\begin{array}{c}\text { All } \\ \text { of the }\end{array} & \begin{array}{c}\text { Most } \\ \text { of the }\end{array} & \begin{array}{c}\text { A Good } \\ \text { Bit of }\end{array} & \begin{array}{c}\text { Some } \\ \text { of the }\end{array} & \begin{array}{c}\text { A Little } \\ \text { of the }\end{array} & \begin{array}{c}\text { None } \\ \text { of the }\end{array} \\ \text { Time } & \underline{\text { Time }} & \underline{\text { the Time }} & \underline{\text { Time }} & \underline{\text { Time }} & \underline{\text { Time }}\end{array}$

a) did you feel full of pep?

1

2

3

4

5

6

b) have you been a very nervous person?

1

2

3 4 5 6

c) have you felt so down in the dumps nothing could cheer you up? 1

2

3

4 5 6

d) have you felt calm and peaceful? 1 2 3 4 5 6

e) did you have a lot of energy? 1 2 3 4 5 6

f) have you felt down hearted and blue? 1 2 3 4 5 6

g) did you feel worn out? 1 2 3 4 5 6

h) have you been a happy person?

1 2 3 4 5 6

i) did you feel tired? 12 3 4 5 6

11. Finally, during the past 4 weeks, how much of the time has your physical health or emotional problems intefered with your social activities (like visiting with friends, relatives, etc.)?

\begin{tabular}{ccccc}
$\begin{array}{c}\text { All of the } \\
\text { time }\end{array}$ & $\begin{array}{c}\text { Most of the } \\
\text { time }\end{array}$ & $\begin{array}{c}\text { Some of the } \\
\text { time }\end{array}$ & $\begin{array}{c}\text { A little of } \\
\text { the time }\end{array}$ & $\begin{array}{c}\text { None of the } \\
\text { time }\end{array}$ \\
\hline 1 & 2 & 3 & 4 & 5
\end{tabular}


Subject's Initials

Study ID \#

Date

\section{PDDS \\ Patient-determined Disease Steps}

Please read the choices listed below and choose the one that best describes your own situation. This scale focuses mainly on how well you walk. Not everyone will find a description that reflects their condition exactly, but please mark the one category that describes your situation the closest.

0 Normal: I may have some mild symptoms, mostly sensory due to MS but they do not limit my activity. If I do have an attack, I return to normal when the attack has passed.

1 Mild Disability: I have some noticeable symptoms from my MS but they are minor and have only a small effect on my lifestyle.

2 Moderate Disability: I don't have any limitations in my walking ability. However, I do have significant problems due to MS that limit daily activities in other ways.

3 Gait Disability: MS does interfere with my activities, especially my walking. I can work a full day, but athletic or physically demanding activities are more difficult than they used to be. I usually don't need a cane or other assistance to walk, but I might need some assistance during an attack.

4 Early Cane: I use a cane or a single crutch or some other form of support (such as touching a wall or leaning on someone's arm) for walking all the time or part of the time, especially when walking outside. I think I can walk 25 feet in 20 seconds without a cane or crutch. I always need some assistance (cane or crutch) if I want to walk as far as 3 blocks.

5 Late Cane: To be able to walk 25 feet, I have to have a cane, crutch or someone to hold onto. I can get around the house or other buildings by holding onto furniture or touching the walls for support. I may use a scooter or wheelchair if I want to go greater distances.

6 Bilateral Support: To be able to walk as far as 25 feet I must have 2 canes or crutches or a walker. I may use a scooter or wheelchair for longer distances.

7 Wheelchair / Scooter: My main form of mobility is a wheelchair. I may be able to stand and/or take one or two steps, but I can't walk 25 feet, even with crutches or a walker.

8 Bedridden: Unable to sit in a wheelchair for more than one hour. 


\section{PERFORMANCE SCALES}

Instructions: These scales are meant to describe some of the different kinds of disabilities people with MS may have. Some of these problems may have to do with MS, and for some you may not be so sure. Do not be concerned by this. We are interested in any problem you have with each aspect of your functioning, regardless of whether it is due to MS, medication or some other health problem. By "normal", we mean the way you were before you developed MS.

\section{MOBILITY}

On the next two pages are categories of mobility disability. Each category has some examples included to help you decide. All of the examples within a category may not apply to you. Please read all of the categories; check the single category that best describes your average condition in the past month. Compare your current condition to your mobility before you developed MS.

$\square 0$ Normal: Functionally normal walking and running.

- Although I have MS and some symptoms, my walking or running is not limited.

1 Minimal Gait Disability: Minor but noticeable effects on mobility.

- I am making minor adjustments in my work or lifestyle because of some difficulty in walking.

- I have given up some particularly strenuous activities because of walking problems.

2 Mild Gait Disability: Noticeable effects on mobility.

- I have to park closer to my destination because of difficulty in walking.

- I have given up some activities, such as long shopping trips, dancing, hiking, or other activities that require a lot of walking.

3 Occasional Use of Cane or Unilateral Support: May use cane or unilateral support (e.g., spouse's arm) outside for greater distances.

- I find myself using the wall, other people's arms, or furniture, to help support my walking at times, but I can walk at least 25 feet (i.e., 2 car lengths) without any support.

- I do not use a cane or similar support around the house, but do use it when I leave the house. I use a cane or similar support to avoid looking like I am drunk.

- I carry a cane even if I do not use it, because it makes me feel more secure. 
4 Frequent Use of Cane: Unable to walk 25 feet (i.e., 2 car lengths) without a cane/unilateral support.

- I need a cane or similar support to get around in the house, as well as outside. I need to use a wall, furniture, or other people's arms to allow me to walk short distances (i.e., less than 25 feet).

- I do not walk anywhere without using some support on one side (e.g., wall, furniture, pushing shopping cart, cane, someone's arm).

5 Severe Gait Disability-Bilateral Support: Requires bilateral support (e.g., crutches, walker) to walk 25 feet (i.e., 2 car lengths).

- I need to hold onto something with both hands in order to walk 25 feet or more.

- I have to use two crutches, walker, or a pushcart in order to walk most of the time.

$\square 6$ Total Gait Disability or Bedridden: Essentially confined to a wheelchair; may be able to take 1 or 2 steps.

- Even though I can take a step or two on my own, I need to use a wheelchair for any distances greater than 25 feet (i.e., 2 car lengths). 


\section{HAND FUNCTION}

Please read all of the categories and check the single category which best describes your worst hand function condition in the past month. Compare your current condition to your hand function before you developed MS.

$\square 0$ Normal: Hands are functionally normal.

- Although I have MS and some symptoms, my hands have not been affected.

\section{Minimal Hand Disability}

- I have some problems with my hands, but it has not changed my activities.

- I do not write as well as I used to.

\section{Mild Hand Disability}

- I am making a few adjustments in my activities because of some difficulty with coordination, shaking, and strength in my hands.

- I have reduced some activities requiring fine hand control, such as writing, handicrafts, typing, etc.

\section{Moderate Hand Disability}

- I am making many adjustments in my activities due to hand problems.

\section{Severe Hand Disability}

- I have given up important activities due to hand problems. I am still able to do my activities, but they take me much longer.

\section{Total Hand Disability}

- Every day, difficulty with my hands prevents me from doing many of my activities. 


\section{VISION}

Please read all of the categories, and check the single category which best describes your overall visual condition (with glasses if you use them) over the past month. Compare your current condition to your vision before you developed MS.

0 Normal Vision: Functionally normal; no limitations on activity or lifestyle.

- MS has not affected my vision.

- I wear glasses but otherwise my vision is normal.

\section{Minimal Visual Disability}

- My vision is normal, but it has been affected in the past by MS.

\section{Mild Visual Disability}

- I have visual symptoms of either blurred or double vision, but I am still able to do all of my usual activities.

\section{Moderate Visual Disability}

- Because of my visual problems, I have been forced to give up some of my usual activities, but after a period of rest I can usually return to these activities.

\section{Severe Visual Disability}

- I am no longer able to maintain my original lifestyle because of my vision, and rest does not seem to help my vision.

- I can no longer drive a car because of my vision.

\section{Total Visual Disability}

- I am essentially blind.

- I cannot read, even with aids. 


\section{FATIGUE}

Please read all of the categories, and check the single category that best describes your fatigue condition in the past month. Compare your current condition to your fatigue level before you developed MS.

$\square 0$ Normal Fatigue: Functionally normal; no limitations on activity or lifestyle.

- I do not notice being fatigued.

\section{Minimal Fatigue Disability}

- I experience fatigue for no apparent reason, but it has not changed my activities.

\section{Mild Fatigue Disability}

- Fatigue occasionally forces me to change some of my activities (e.g., once a week or less).

\section{Moderate Fatigue Disability}

- Fatigue frequently forces me to change some of my activities (e.g., several times a week).

\section{Severe Fatigue Disability}

- Every day, fatigue forces me to modify my daily activities.

- I am always tired.

\section{Total Fatigue Disability}

- Every day, fatigue prevents me from doing many of my daily activities. 
Study ID \#

\section{COGNITIVE SYMPTOMS}

Problems with remembering, thinking, difficulties with calculations, confusion, difficulty remembering what you read, word recall, etc., compared to before you developed MS.

Please read all of the categories, and check the single category which best describes your cognitive symptoms in the past month. Compare your current condition to your level of cognition before you developed MS.

$\square 0$ Normal Cognition: Functionally normal; no limitations on activity or lifestyle.

- I have not noticed any problems with memory or confusion.

$\square 1$ Minimal Cognitive Disability

- I notice some problems with memory or confusion, but they do not interfere with my activities.

\section{Mild Cognitive Disability}

- Memory problems or confusion occasionally affect some of my activities (e.g., once a week or less).

\section{Moderate Cognitive Disability}

Memory problems or confusion frequently affect some of my activities (e.g., several times a week).

\section{Severe Cognitive Disability}

- I constantly need to allow for problems with memory or confusion in my daily activities.

\section{Total Cognitive Disability}

- Every day, memory problems or confusion prevent me from doing many of my daily activities. 


\section{BLADDER / BOWEL}

Please read all of the categories, and check the single category which best describes your bladder or bowel symptoms in the past month even if you use a catheter or have had surgical procedures. Compare your current condition to your bladder/bowel function before you developed MS.

$\square 0$ Normal Bladder/Bowel: Functionally normal; no limitations on activity or lifestyle.

- I have not noticed any problems with my bladder or bowel control.

\section{$\square 1$ Minimal Bladder/Bowel Disability}

- I have some problems with bladder or bowel control (e.g., urinary frequency, urgency or hesitancy), but it does not interfere with my activities.

- I am aware of needing to control my bladder and bowel, and I do not have any problem with wetting or soiling.

\section{Mild Bladder/Bowel Disability}

- Bladder or bowel control problems occasionally affect some of my activities (e.g., once a week or less).

- I have trouble controlling my bladder or bowel once a week or less (e.g., dribbling).

\section{Moderate Bladder/Bowel Disability}

- Bladder or bowel control problems frequently affect some of my activities (e.g., several times a week). I have trouble controlling my bladder or bowel several times a week (e.g., wet or soil).

\section{Severe Bladder/Bowel Disability}

- Every day, bladder or bowel control problems force me to modify my daily activities.

\section{Total Bladder/Bowel Disability}

- Every day, bladder or bowel control problems prevent me from doing many of my daily activities. 


\section{SENSORY SYMPTOMS Study ID \# \\ SENSORY SYMPTOMS}

Problems with numbness, tingling, odd sensations, electric shock, burning, band-like sensation around trunk or extremities. Do not include aching pain or headaches.

Please read all of the categories, and check the single category that best describes your sensory symptoms in the past month. Compare your current condition to your level of sensory function before you developed MS.

0 Normal Sensory: Functionally normal; no limitations on activity or lifestyle.

- I have not noticed any problems with numbness or tingling.

\section{Minimal Sensory Disability}

- I have some problems with numbness or tingling, but it does not interfere with my activities.

\section{Mild Sensory Disability}

- Numbness or tingling occasionally forces me to change some of my activities (e.g., once a week or less).

\section{Moderate Sensory Disability}

- Numbness or tingling frequently affects some of my activities (e.g., several times a week).

\section{Severe Sensory Disability}

- Every day, numbness or tingling problems force me to modify my daily activities.

\section{Total Sensory Disability}

- Every day, numbness or tingling prevents me from doing many of my daily activities. 


\section{SPASTICITY SYMPTOMS}

Unusual tightening of muscles that feels like leg stiffness, jumping of legs, a repetitive bouncing of the foot, muscle cramping in legs or arms or legs going out tight and straight or drawing up.

Please read all the categories, and check the single category that most accurately describes your spasticity symptoms in the past month. Compare your current condition to your level of spasticity before you developed MS.

0 Normal Spasticity: No symptoms of spasticity.

- I have not noticed any problems with spasticity.

$\square 1$ Minimal Spasticity Disability

- I notice some problems with spasticity, but they do not interfere with my activities.

\section{Mild Spasticity Disability}

- Spasticity occasionally forces me to change some of my activities (e.g., once a week or less).

\section{Moderate Spasticity Disability}

- Spasticity frequently affects some of my activities (e.g., several times a week).

\section{Severe Spasticity Disability}

- Every day, spasticity problems force me to modify my daily activities.

\section{Total Spasticity Disability}

- Every day, spasticity problems prevent me from doing many of my daily activities. 


\section{PAIN}

Please read all the categories, and check the single category that most accurately describes your pain (regardless of cause) in the past month. Compare your current condition to your experience before you developed MS.

$\square 0$ Normal: No symptoms of pain.

- I have not noticed any problems with pain.

$\square 1$ Minimal Pain

- I notice some problems with pain, but they do not interfere with my activities.

\section{Mild Pain}

- Pain occasionally forces me to change some of my activities (e.g., once a week or less).

\section{Moderate Pain}

- Pain frequently affects some of my activities (e.g., several times a week).

\section{Severe Pain}

- Every day, pain problems force me to modify my daily activities.

\section{Total Disabling Pain}

- Every day, pain problems prevent me from doing many of my daily activities. 


\section{DEPRESSION}

Please read all the categories, and check the single category which most accurately describes symptoms of depression in the past month. Compare your current condition to what you felt before you developed MS.

$\square 0$ Normal: No symptoms of depression.

- I have not noticed any problems with depression.

$\square 1$ Minimal Depression

- I notice some problems with depression, but they do not interfere with my activities.

\section{Mild Depression}

- Depression occasionally forces me to change some of my activities (e.g., once a week or less).

\section{$\square 3$ Moderate Depression}

- Depression frequently affects some of my activities (e.g., several times a week).

\section{Severe Depression}

- Every day, depression problems force me to modify my daily activities.

\section{$\square 5$ Total Depression}

- Every day, depression problems prevent me from doing many of my daily activities. 
Study ID \#

\section{TREMOR / LOSS OF COORDINATION}

Tremor is the rhythmic shaking of the head, hands or legs. Loss of coordination is clumsiness or imbalance (e.g., staggering gait or unsteady gait like being drunk).

Please read all the categories, and check the single category that most accurately describes your tremor and loss of coordination symptoms in the past month. Compare your current condition to your experience before you developed MS.

$\square 0 \quad$ Normal/no tremor or loss of coordination

- I do not have any tremor or loss of coordination.

- I have not noticed any problems with tremor or loss of coordination.

\section{Minimal tremor or loss of coordination}

- Sometimes I have some tremor or loss of coordination, but it does not interfere with my activities.

2 Mild tremor or loss of coordination

- Tremor or loss of coordination occasionally forces me to change some of my activities (e.g., once a week or less).

\section{Moderate tremor or loss of coordination}

Tremor or loss of coordination frequently affects some of my activities (e.g., several times a week).

\section{Severe tremor or loss of coordination}

- Every day, tremor or loss of coordination problems force me to modify my daily activities.

\section{Total Disabling tremor or loss of coordination}

- Every day, tremor or loss of coordination problems prevent me from doing many of my daily activities. 
Subject Initials:

Study \#:

Visit Number:

\section{Modified Ashworth Scale for grading Spasticity}

\begin{tabular}{|l|l|}
\hline Grade & Description \\
\hline 0 & No increase in muscle tone \\
\hline 1 & $\begin{array}{l}\text { Slight increase in muscle tone, } \\
\text { manifested by a catch and release, or by } \\
\text { minimal resistance at the end of the } \\
\text { range of motion when the affected part(s) } \\
\text { is moved in flexion or extension }\end{array}$ \\
\hline 2 & $\begin{array}{l}\text { Slight increase in muscle tone, } \\
\text { manifested by a catch, followed by } \\
\text { minimal resistance throughout the } \\
\text { remainder (less than half) of the range of } \\
\text { movement (ROM) }\end{array}$ \\
\hline 3 & $\begin{array}{l}\text { More marked increase in muscle tone } \\
\text { through most of ROM, but affected } \\
\text { part(s) easily moved }\end{array}$ \\
\hline 4 & $\begin{array}{l}\text { Considerable increase in muscle tone, } \\
\text { passive movement difficult }\end{array}$ \\
\hline 5 & $\begin{array}{l}\text { Affected part(s) rigid in flexion and } \\
\text { extension }\end{array}$ \\
\hline
\end{tabular}

SCORING TABLE

\begin{tabular}{|c|c|}
\hline Tested Limb & Score \\
\hline Right Upper Extremity & \\
\hline Right Lower Extremity & \\
\hline Left Upper Extremity & \\
\hline Left Lower Extremity & \\
\hline
\end{tabular}




\section{Bibliography}

[1] C. L. Vaughan, B. L. Davis, and J. C. O'Connor, Dynamics of human gait. Human Kinetics Publishers Champaign, Illinois, 1992, vol. 2.

[2] J. D. Haines, M. Inglese, and P. Casaccia, "Axonal damage in multiple sclerosis," Mount Sinai Journal of Medicine: A Journal of Translational and Personalized Medicine, vol. 78, no. 2, pp. 231-243, 2011.

[3] "MS multiple sclerosis." [Online]. Available: http://www.physio-pedia.com/

[4] H. L. Zwibel, "Contribution of impaired mobility and general symptoms to the burden of multiple sclerosis," Advances in Therapy, vol. 26, no. 12, pp. 10431057, Dec 2009.

[5] C. H. Polman, S. C. Reingold, B. Banwell, M. Clanet, J. A. Cohen, M. Filippi, K. Fujihara, E. Havrdova, M. Hutchinson, L. Kappos, et al., "Diagnostic criteria for multiple sclerosis: 2010 revisions to the McDonald criteria," Annals of Neurology, vol. 69, no. 2, pp. 292-302, 2011.

[6] "Atlas. Multiple sclerosis resources in the world, 2008," World Health Organization, 2008.

[7] J. F. Kurtzke, "Rating neurologic impairment in multiple sclerosis: an expanded disability status scale (EDSS)," Neurology, vol. 33, no. 11, pp. 1444-1452, 1983. 
[8] M. D. Goldman, R. A. Marrie, and J. A. Cohen, "Evaluation of the six-minute walk in multiple sclerosis subjects and healthy controls," Multiple Sclerosis, vol. 14, no. 3, pp. 383-390, Apr 2008.

[9] R. Butland, J. Pang, E. Gross, A. Woodcock, and D. Geddes, "Two-, six-, and 12-minute walking tests in respiratory disease." British Medical Journal (Clinical Research ed.), vol. 284, no. 6329, pp. 1607-1608, 1982.

[10] P. G. Ritvo, J. S. Fischer, D. M. Miller, H. Andrews, D. Paty, and N. LaRocca, "Multiple sclerosis quality of life inventory: a user's manual," New York: National Multiple Sclerosis Society, pp. 1-65, 1997.

[11] B. C. Kieseier and C. Pozzilli, "Assessing walking disability in multiple sclerosis," Multiple Sclerosis Journal, vol. 18, no. 7, pp. 914-924, 2012.

[12] Y. Li and C. E. Schwartz, "Data mining for response shift patterns in multiple sclerosis patients using recursive partitioning tree analysis," Quality of Life Research, vol. 20, no. 10, pp. 1543-1553, 2011.

[13] A. K. Bourke, F. Masse, A. Arami, K. Aminian, M. Healy, J. Nelson, C. O'Dwyer, and S. Coote, "Energy expenditure estimation using accelerometry and heart rate for multiple sclerosis and healthy older adults," in Wearable and Implantable Body Sensor Networks Workshops (BSN Workshops), 11th Int. Conf. IEEE, 2014, pp. 1-5.

[14] J. Gong, J. Lach, Y. Qi, and M. D. Goldman, "Causal analysis of inertial body sensors for enhancing gait assessment separability towards multiple sclerosis diagnosis," in Wearable and Implantable Body Sensor Networks (BSN), 12th Int. Conf. IEEE, 2015, pp. 1-6. 
[15] A. Shrinivasan, "Classification of gait in patients with normal pressure hydrocephalus," Master's thesis, University of Virginia, 2011.

[16] S. Chen, A. T. Barth, J. T. Barth, B. C. Bennett, M. Brandt-Pearce, D. K. Broshek, J. R. Freeman, H. L. Samples, and J. Lach, "Aiding diagnosis of normal pressure hydrocephalus with enhanced gait feature separability," in Proc. of the Conf. on Wireless Health. ACM, 2012, p. 3.

[17] M. M. Engelhard, S. R. Dandu, S. D. Patek, J. C. Lach, and M. D. Goldman, "Quantifying six-minute walk induced gait deterioration with inertial sensors in multiple sclerosis subjects," Gait \& Posture, vol. 49, pp. 340-345, 2016.

[18] N. Nowaczyk and L. Cierpiałkowska, "Psychological profiles of patients with multiple sclerosis based on Hobfoll's conservation of resources theory," Health Psychol Rep, 2016.

[19] R. LeMoyne, F. Heerinckx, T. Aranca, R. De Jager, T. Zesiewicz, and H. J. Saal, "Wearable body and wireless inertial sensors for machine learning classification of gait for people with Friedreich's ataxia," in Wearable and Implantable Body Sensor Networks (BSN), 2016 IEEE 13th Int. Conf. on. IEEE, 2016, pp. $147-151$.

[20] J. M. Hausdorff, M. E. Cudkowicz, R. Firtion, J. Y. Wei, and A. L. Goldberger, "Gait variability and basal ganglia disorders: Stride-to-stride variations of gait cycle timing in Parkinson's disease and Huntington's disease," Movement Disorders, vol. 13, no. 3, pp. 428-437, 1998.

[21] E. Sejdic, K. A. Lowry, J. Bellanca, M. S. Redfern, and J. S. Brach, "A comprehensive assessment of gait accelerometry signals in time, frequency and time- 
frequency domains," IEEE Transactions on Neural Systems and Rehabilitation Engineering, vol. 22, no. 3, pp. 603-612, 2014.

[22] D. Maquet, F. Lekeu, E. Warzee, S. Gillain, V. Wojtasik, E. Salmon, J. Petermans, and J.-L. Croisier, "Gait analysis in elderly adult patients with mild cognitive impairment and patients with mild Alzheimer's disease: simple versus dual task: a preliminary report," Clinical Physiology and Functional Imaging, vol. 30, no. 1, pp. 51-56, 2010.

[23] A. Shrinivasan, M. Brandt-Pearce, A. Barth, and J. Lach, "Analysis of gait in patients with normal pressure hydrocephalus," in Proc. of the First ACM Workshop on Mobile Systems, Applications, and Services for Healthcare. ACM, 2011, p. 3.

[24] C. Mizuike, S. Ohgi, and S. Morita, "Analysis of stroke patient walking dynamics using a tri-axial accelerometer," Gait \& Posture, vol. 30, no. 1, pp. 60-64, 2009.

[25] A. L. Goldberger, L. A. Amaral, J. M. Hausdorff, P. C. Ivanov, C.-K. Peng, and H. E. Stanley, "Fractal dynamics in physiology: alterations with disease and aging," Proc. of the National Academy of Sciences, vol. 99, no. suppl 1, pp. 2466-2472, 2002.

[26] N. E. Huang, Z. Shen, S. R. Long, M. C. Wu, H. H. Shih, Q. Zheng, N.-C. Yen, C. C. Tung, and H. H. Liu, "The empirical mode decomposition and the Hilbert spectrum for nonlinear and non-stationary time series analysis," in Proc. of the Royal Society of London A: Mathematical, Physical and Engineering Sciences, vol. 454, no. 1971. The Royal Society, 1998, pp. 903-995. 
[27] T. Hastie, R. Tibshirani, and J. Friedman, The Elements of Statistical Learning Data Mining, Inference, and Prediction. Springer, 2008.

[28] D. J. Hand, H. Mannila, and P. Smyth, Principles of data mining. MIT press, 2001.

[29] C. Ahlrichs, A. Samà, M. Lawo, J. Cabestany, D. Rodríguez-Martín, C. PérezLópez, D. Sweeney, L. R. Quinlan, G. Ò. Laighin, T. Counihan, et al., "Detecting freezing of gait with a tri-axial accelerometer in Parkinson's disease patients," Medical \& Biological Engineering $\&$ Computing, vol. 54, no. 1, pp. 223-233, 2016.

[30] S. Chen, C. L. Cunningham, B. C. Bennett, and J. Lach, "Enabling longitudinal assessment of ankle-foot orthosis efficacy for children with cerebral palsy," in Proc. of the 2nd Conf. on Wireless Health. ACM, 2011, p. 4.

[31] L. Ada, C. M. Dean, J. M. Hall, J. Bampton, and S. Crompton, "A treadmill and overground walking program improves walking in persons residing in the community after stroke: a placebo-controlled, randomized trial," Archives of Physical Medicine and Rehabilitation, vol. 84, no. 10, pp. 1486-1491, 2003.

[32] S. Chen, C. L. Cunningham, J. Lach, and B. C. Bennett, "Extracting spatiotemporal information from inertial body sensor networks for gait speed estimation," in Body Sensor Networks (BSN), Int. Conf. IEEE, 2011, pp. 71-76.

[33] L. Atallah, G. G. Jones, R. Ali, J. J. Leong, B. Lo, and G.-Z. Yang, "Observing recovery from knee-replacement surgery by using wearable sensors," in Body Sensor Networks (BSN), 2011 Int. Conf. on. IEEE, May 2011, pp. 29-34. 
[34] G. Chen, C. Patten, D. H. Kothari, and F. E. Zajac, "Gait differences between individuals with post-stroke hemiparesis and non-disabled controls at matched speeds," Gait \&3 Posture, vol. 22, no. 1, pp. 51-56, 2005.

[35] A. T. Barth, B. Boudaoud, J. S. Brantley, S. Chen, C. L. Cunningham, T. Kim, H. C. Powell Jr, S. A. Ridenour, J. Lach, and B. C. Bennett, "Longitudinal highfidelity gait analysis with wireless inertial body sensors," in Wireless Health. ACM, 2010, pp. 192-193.

[36] J. Gong, P. Asare, J. Lach, and Y. Qi, "Piecewise linear dynamical model for actions clustering from inertial body sensors with considerations of human factors," in Proc. of the 9th Int. Conf. on Body Area Networks. ICST (Institute for Computer Sciences, Social-Informatics and Telecommunications Engineering), 2014, pp. 90-96.

[37] M. Mancini, L. King, A. Salarian, L. Holmstrom, J. McNames, and F. B. Horak, "Mobility Lab to assess balance and gait with synchronized body-worn sensors," Journal of Bioengineering \& Biomedical Science, p. 007, 2012.

[38] L. Li, L. Atallah, B. Lo, and G.-Z. Yang, "Feature extraction from ear-worn sensor data for gait analysis," in Biomedical and Health Informatics (BHI), EMBS Int. Conf. IEEE, 2014, pp. 560-563.

[39] C. E. Garber and J. H. Friedman, "Effects of fatigue on physical activity and function in patients with Parkinson's disease," Neurology, vol. 60, no. 7, pp. 1119-1124, 2003.

[40] S. Savci, D. Inal-Ince, H. Arikan, A. Guclu-Gunduz, N. Cetisli-Korkmaz, K. Armutlu, and R. Karabudak, "Six-minute walk distance as a measure of functional 
exercise capacity in multiple sclerosis," Disability and Rehabilitation, vol. 27, no. 22 , pp. 1365-1371, 2005.

[41] A. Chetta, A. Rampello, E. Marangio, S. Merlini, F. Dazzi, M. Aiello, F. Ferraro, A. Foresi, M. Franceschini, and D. Olivieri, "Cardiorespiratory response to walk in multiple sclerosis patients," Respiratory Medicine, vol. 98, no. 6, pp. $522-529,2004$.

[42] C. M. Dean, C. L. Richards, and F. Malouin, "Task-related circuit training improves performance of locomotor tasks in chronic stroke: a randomized, controlled pilot trial," Archives of Physical Medicine and Rehabilitation, vol. 81, no. 4, pp. 409-417, 2000.

[43] J. J. Daly, K. Roenigk, J. Holcomb, J. M. Rogers, K. Butler, J. Gansen, J. McCabe, E. Fredrickson, E. B. Marsolais, and R. L. Ruff, "A randomized controlled trial of functional neuromuscular stimulation in chronic stroke subjects," Stroke, vol. 37, no. 1, pp. 172-178, 2006.

[44] P. Duncan, S. Studenski, L. Richards, S. Gollub, S. M. Lai, D. Reker, S. Perera, J. Yates, V. Koch, S. Rigler, et al., "Randomized clinical trial of therapeutic exercise in subacute stroke," Stroke, vol. 34, no. 9, pp. 2173-2180, 2003.

[45] U. Givon, G. Zeilig, and A. Achiron, "Gait analysis in multiple sclerosis: characterization of temporal-spatial parameters using GAITRite functional ambulation system," Gait $\&$ Posture, vol. 29, no. 1, pp. 138-142, 2009.

[46] C. L. Martin, B. Phillips, T. Kilpatrick, H. Butzkueven, N. Tubridy, E. McDonald, and M. Galea, "Gait and balance impairment in early multiple sclerosis in the absence of clinical disability," Multiple Sclerosis, vol. 12, no. 5, pp. 620-628, 2006. 
[47] J. Gong, M. M. Engelhard, M. D. Goldman, and J. Lach, "Correlations between inertial body sensor measures and clinical measures in multiple sclerosis," in Proc. of the 10th EAI Int. Conf. on Body Area Networks, 2015, pp. 18-24.

[48] J. Hobart, A. Riazi, D. Lamping, R. Fitzpatrick, and A. Thompson, "Measuring the impact of MS on walking ability the 12-item MS walking scale (MSWS-12)," Neurology, vol. 60, no. 1, pp. 31-36, Jan 2003.

[49] S. R. Dandu, M. M. Engelhard, M. D. Goldman, and J. Lach, "Determining physiological significance of inertial gait features in multiple sclerosis," in Wearable and Implantable Body Sensor Networks (BSN), 2016 IEEE 13th Int. Conf. on. IEEE, 2016, pp. 266-271.

[50] M. Pau, S. Caggiari, A. Mura, F. Corona, B. Leban, G. Coghe, L. Lorefice, M. G. Marrosu, and E. Cocco, "Clinical assessment of gait in individuals with multiple sclerosis using wearable inertial sensors: Comparison with patientbased measure," Multiple Sclerosis and Related Disorders, vol. 10, pp. 187-191, 2016.

[51] S. Crenshaw, T. Royer, J. Richards, and D. Hudson, "Gait variability in people with multiple sclerosis," Multiple Sclerosis, vol. 12, no. 5, pp. 613-619, Sep 2006.

[52] R. W. Motl, D. Dlugonski, Y. Suh, M. Weikert, B. Fernhall, and G. M, "Accelerometry and its association with objective markers of walking limitations in ambulatory adults with multiple sclerosis," Archives of Physical Medicine and Rehabilitation, vol. 91, no. 12, pp. 1942-1947, 2010.

[53] T. Sandmann, "Relevant biomarkers in the prediction of good and bad days for multiple sclerosis patients," Bachelor Thesis, Radboud University, 2016. 
[54] M. J. Socie, M. K. Boes, and B. M. Sandroff, "Does a waist-worn Actigraph accelerometer quantify community ambulation in persons with multiple sclerosis?" Journal of Rehabilitation Research and Development, vol. 49, no. 9, p. $1405,2012$.

[55] R. I. Spain, M. Mancini, F. B. Horak, and D. Bourdette, "Body-worn sensors capture variability, but not decline, of gait and balance measures in multiple sclerosis over 18 months," Gait \&3 Posture, vol. 39, no. 3, pp. 958-964, 2014.

[56] J. M. Huisinga, M. Mancini, R. J. S. George, and F. B. Horak, "Accelerometry reveals differences in gait variability between patients with multiple sclerosis and healthy controls," Annals of Biomedical Engineering, vol. 41, no. 8, pp. 1670-1679, Aug 2013.

[57] M. M. Engelhard, J. C. Lach, K. M. Schmidt, M. D. Goldman, and S. D. Patek, "Adaptive symptom reporting for mobile patient-reported disability assessment," in 2016 IEEE Wireless Health (WH), 2016, pp. 1-8.

[58] J. M. Hausdorff, "Gait dynamics, fractals and falls: finding meaning in the stride-to-stride fluctuations of human walking," Human Movement Science, vol. 26, no. 4, pp. 555-589, Aug 2007.

[59] H. Miller, C. A. Simpson, and W. K. Yeates, "Bladder dysfunction in multiple sclerosis," British Medical Journal, vol. 1, no. 5445, pp. 1265-1269, May 1965.

[60] M. Alaqtash, T. Sarkodie-Gyan, H. Yu, O. Fuentes, R. Brower, and A. Abdelgawad, "Automatic classification of pathological gait patterns using ground reaction forces and machine learning algorithms," in Engineering in Medicine and Biology Society, EMBC, 2011 Annual Int. Conf. of the IEEE. IEEE, 2011, pp. $453-457$. 
[61] J. Han, R. B. Altman, V. Kumar, H. Mannila, and D. Pregibon, "Emerging scientific applications in data mining," Communications of the ACM, vol. 45, no. 8 , pp. 54-58, 2002.

[62] S. Kropp and V. Caulfield, "Data mining and bioinformatics," Monash University Faculty of Information Technology Caulfield, VIC, 2004.

[63] P. Bonato, D. M. Sherrill, D. G. Standaert, S. S. Salles, and M. Akay, "Data mining techniques to detect motor fluctuations in Parkinson's disease," in Engineering in Medicine and Biology Society, 2004. IEMBS 2004. 26th Annual Int. Conf. of the IEEE, vol. 2. IEEE, 2004, pp. 4766-4769.

[64] R. G. Ramani and G. Sivagami, "Parkinson disease classification using data mining algorithms," International Journal of Computer Applications, vol. 32, no. 9, pp. 17-22, 2011.

[65] B. Bejarano, M. Bianco, D. Gonzalez-Moron, J. Sepulcre, J. Goñi, J. Arcocha, O. Soto, U. Del Carro, G. Comi, L. Leocani, et al., "Computational classifiers for predicting the short-term course of multiple sclerosis," BMC Neurology, vol. 11, no. 1, p. $67,2011$.

[66] J. Tobler, M. Molla, E. F. Nuwaysir, R. Green, and J. W. Shavlik, "Evaluating machine learning approaches for aiding probe selection for gene-expression arrays," Bioinformatics, vol. 18, no. suppl 1, pp. S164-S171, 2002.

[67] J. Taylor, R. D. King, T. Altmann, and O. Fiehn, "Application of metabolomics to plant genotype discrimination using statistics and machine learning," Bioinformatics, vol. 18, no. Suppl. 2, pp. S241-S248, 2002. 
[68] J. Li, H. Liu, S.-K. Ng, and L. Wong, "Discovery of significant rules for classifying cancer diagnosis data," Bioinformatics, vol. 19, no. Suppl. 2, pp. ii93-ii102, 2003.

[69] E. Kretschmann, W. Fleischmann, and R. Apweiler, "Automatic rule generation for protein annotation with the c4. 5 data mining algorithm applied on SWISSPROT," Bioinformatics, vol. 17, no. 10, pp. 920-926, 2001.

[70] J. Parkinson, D. Muthas, M. Clark, S. Boyer, J.-P. Valentin, and L. Ewart, "Application of data mining and visualization techniques for the prediction of drug-induced nausea in man," Toxicological Sciences, vol. 126, no. 1, pp. 275284, 2012.

[71] A. Qureshi and M. Brandt-Pearce, "On modified EMD: Selective extrema analysis," in Signal Processing Systems (SiPS). IEEE, 2014, pp. 1-6.

[72] C.-K. Peng, J. M. Hausdorff, and A. L. Goldberger, "Fractal mechanisms in neural control: Human heartbeat and gait dynamics in health and disease," Self-Organized Biological Dynamics and Nonlinear Control, J. Walleczek, 2000.

[73] S. Qian, Introduction to Time-Frequency and Wavelet Transforms. Prentice Hall Inc., Upper Saddle River, NJ, 2002.

[74] L. Cohen, Time-Frequency Analysis. Prentice Hall PTR, Upper Saddle River, NJ, 1995, vol. 778 .

[75] S. Tolwinski, "The Hilbert transform and empirical mode decomposition as tools for data analysis," Tucson: University of Arizona, 2007.

[76] V. Vatchev and R. Sharpley, "Decomposition of functions into pairs of intrinsic mode functions," in Proc. of the Royal Society of London A: Mathematical, 
Physical and Engineering Sciences, vol. 464, no. 2097. The Royal Society, 2008, pp. 2265-2280.

[77] N.E. Huang et al., "On the establishment of a confidence limit for the empirical mode decomposition and Hilbert spectral analysis," Proc. of the Royal Society of London. Series A: Mathematical, Physical and Engineering Sciences, vol. 459, pp. 2317-2345, 2003.

[78] D. Kim and H.-S. Oh, "EMD: A package for empirical mode decomposition and Hilbert spectrum," The R Journal, vol. 1, no. 1, pp. 40-46, 2009.

[79] N. Wang, E. Ambikairajah, B. G. Celler, and N. H. Lovell, "Feature extraction using an AM-FM model for gait pattern classification," in Biomedical Circuits and Systems Conf., BioCAS. IEEE, 2008, pp. 25-28.

[80] P. Kuchi and S. Panchanathan, "Intrinsic mode functions for gait recognition," in Circuits and Systems, ISCAS. Proc. of the 2004 Int. Symposium, vol. 2. IEEE, 2004, pp. II-117.

[81] K. Schiecke, D. Piper, S. Buerger, L. Leistritz, M. Feucht, and H. Witte, "Empirical mode decomposition of heart rate variability. a methodological study," in Cardiovascular Oscillations (ESGCO), 8th Conf. of the European Study Group. IEEE, 2014, pp. 105-106.

[82] Z. Qingjie, Z. Huayong, and S. Lincheng, "A new method for mitigation of end effect in empirical mode decomposition," in Informatics in Control, Automation and Robotics (CAR), 2nd Int. Asia Conf., vol. 1. IEEE, 2010, pp. 400-403.

[83] B. L. Barnhart, "The Hilbert-Huang transform: theory, applications, development," Ph.D. dissertation, University of Iowa, 2011. 
[84] J. Yan and L. Lu, "Improved Hilbert-Huang transform based weak signal detection methodology and its application on incipient fault diagnosis and ECG signal analysis," Signal Processing, vol. 98, pp. 74-87, 2014.

[85] R. K. Ibrahim, E. Ambikairajah, B. G. Celler, and N. H. Lovell, "Gait pattern classification using compact features extracted from intrinsic mode functions," in Engineering in Medicine and Biology Society (EMBS). 30th Annual Int. Conf. IEEE, 2008, pp. 3852-3855.

[86] M.-H. Yeh, "The complex bidimensional empirical mode decomposition," Signal Processing, vol. 92, no. 2, pp. 523-541, 2012.

[87] B. Huang and A. Kunoth, "An optimization based empirical mode decomposition scheme," Journal of Computational and Applied Mathematics, vol. 240, pp. 174-183, Mar 2013.

[88] T. Lee, S. Sanei, and M. Belkhatir, "Combining biometrics derived from different classes of nonlinear analyses of fronto-normal gait signals," Int. Journal on Advances in Networks and Services, vol. 4, no. 1 \& 2, 2011.

[89] A. Bouchikhi and A.-O. Boudraa, "Multicomponent AM-FM signals analysis based on EMD-B-splines ESA," Signal Processing, vol. 92, no. 9, pp. 2214-2228, 2012 .

[90] A. Cicone, J. Liu, and H. Zhou, "Adaptive local iterative filtering for signal decomposition and instantaneous frequency analysis," Applied and Computational Harmonic Analysis, vol. 41, no. 2, pp. 384-411, 2016.

[91] L. Lin, Y. Wang, and H. Zhou, "Iterative filtering as an alternative algorithm for empirical mode decomposition," Advances in Adaptive Data Analysis, vol. 1, no. 04 , pp. 543-560, 2009. 
[92] R. A. Marrie and M. Goldman, "Validity of performance scales for disability assessment in multiple sclerosis," Multiple Sclerosis, 2007.

[93] S. D. McPhee, "Functional hand evaluations: a review," American Journal of Occupational Therapy, vol. 41, no. 3, pp. 158-163, 1987.

[94] T. Platz, C. Eickhof, G. Nuyens, and P. Vuadens, "Clinical scales for the assessment of spasticity, associated phenomena, and function: a systematic review of the literature," Disability and Rehabilitation, vol. 27, no. 1-2, pp. 7-18, 2005.

[95] "Twelve item MS walking scale (MSWS-12)." [Online]. Available: http://download.lww.com/wolterskluwer_vitalstream_com/PermaLink/ JNPT/A/JNPT_41_1_2016_10_28_MELZER_JNPT-D-15-00077R3_SDC2.pdf

[96] J. Hobart, J. Freeman, D. Lamping, R. Fitzpatrick, and A. Thompson, "The SF-36 in multiple sclerosis: why basic assumptions must be tested," Journal of Neurology, Neurosurgery \& Psychiatry, vol. 71, no. 3, pp. 363-370, 2001.

[97] J. Hobart, D. Lamping, R. Fitzpatrick, A. Riazi, and A. Thompson, "The multiple sclerosis impact scale (MSIS-29)," Brain, vol. 124, no. 5, pp. 962-973, 2001.

[98] M. Hohol, E. Orav, and H. Weiner, "Disease steps in multiple sclerosis a simple approach to evaluate disease progression," Neurology, vol. 45, no. 2, pp. 251$255,1995$.

[99] S. R. Dandu, M. M. Engelhard, A. Qureshi, J. Gong, J. C. Lach, M. BrandtPearce, and M. D. Goldman, "Understanding the physiological significance of four inertial gait features in multiple sclerosis," Journal of Biomedical and Health Informatics, Special issue, 2017, (submitted). 
[100] J. M. Hausdorff, G. Yogev, S. Springer, E. S. Simon, and N. Giladi, "Walking is more like catching than tapping: gait in the elderly as a complex cognitive task," Experimental Brain Research, vol. 164, no. 4, pp. 541-548, 2005.

[101] A. T. Barth, M. Hanson, H. C. Powell Jr, J. Lach, et al., "Tempo 3.1: A body area sensor network platform for continuous movement assessment," in Wearable and Implantable Body Sensor Networks, BSN. Sixth Int. Workshop. IEEE, 2009, pp. 71-76.

[102] A. Mostayed, M. Mynuddin, G. Mazumder, S. Kim, and S. J. Park, "Abnormal gait detection using discrete Fourier transform," in Multimedia and Ubiquitous Engineering, MUE. Int. Conf. IEEE, 2008, pp. 36-40.

[103] H. C. Powell, M. A. Hanson, and J. Lach, "A wearable inertial sensing technology for clinical assessment of tremor," in Biomedical Circuits and Systems Conf., BIOCAS. IEEE, 2007, pp. 9-12.

[104] J. K. Loudon, "Biomechanics of gait and running," Medicine and Science in Sports and Exercise, pp. 1-2, 2000.

[105] "Gait." [Online]. Available: http://www.physio-pedia.com/Gait

[106] Q. Li, M. Young, V. Naing, and J. Donelan, "Walking speed and slope estimation using shank-mounted inertial measurement units," in Rehabilitation Robotics, 2009. ICORR 2009. IEEE Int. Conf. on. IEEE, 2009, pp. 839-844.

[107] M. Hollander, D. A. Wolfe, and E. Chicken, Nonparametric statistical methods. John Wiley \& Sons, 2013. 
[108] J. H. Zar, "Significance testing of the spearman rank correlation coefficient," Journal of the American Statistical Association, vol. 67, no. 339, pp. 578-580, 1972.

[109] R. L. Wasserstein and N. A. Lazar, "The ASA's statement on p-values: context, process, and purpose," Am Stat, vol. 70, no. 2, pp. 129-133, 2016.

[110] N. R. Draper, H. Smith, and E. Pownell, Applied regression analysis. Wiley New York, 1966, vol. 3.

[111] H. Akaike, "Information theory and an extension of the maximum likelihood principle," in Selected Papers of Hirotugu Akaike. Springer, 1998, pp. 199-213.

[112] M. D. Goldman, R. A. Marrie, and J. A. Cohen, "Evaluation of the six-minute walk in multiple sclerosis subjects and healthy controls," Multiple Sclerosis, vol. 14, no. 3, pp. 383-390, Apr 2008.

[113] R. G. D. Steel and J. H. Torrie, "Principles and procedures of statistics; with special reference to the biological sciences," 1960.

[114] A. Qureshi, M. Brandt-Pearce, and M. D. Goldman, "Relationship between gait variables and domains of neurologic dysfunction in multiple sclerosis using 6-minute walk test," in The IEEE Engineering in Medicine and Biology Society, 2016 Int. Conf. on. IEEE, Aug 2016, pp. 1-4.

[115] A. Qureshi, M. Brandt-Pearce, M. M. Engelhard, and M. D. Goldman, "Relationship between kernel density function estimates of gait time series and clinical data," in Biomedical and Health Informatics (BHI), EMBS Int. Conf. IEEE, 2017. 
[116] A. Qureshi, M. Brandt-Pearce, and M. D. Goldman, "Longitudinal estimation of gait time series density in multiple sclerosis subjects using inertial data," in 2016 IEEE 13th Int. Conf. on Wearable and Implantable Body Sensor Networks (BSN). IEEE, 2016, pp. 152-152.

[117] A. Qureshi, M. M. Engelhard, M. Brandt-Pearce, and M. D. Goldman, "Demonstrating the real-world significance of the mid-swing to heel strike part of the gait cycle using spectral features," in 2017 IEEE 14th Int. Conf. on Wearable and Implantable Body Sensor Networks (BSN). IEEE, 2017.

[118] M. A. Oskoei, H. Hu, and J. Q. Gan, "Manifestation of fatigue in myoelectric signals of dynamic contractions produced during playing PC games," in Engineering in Medicine and Biology Society. EMBS 2008. 30th Annual Int. Conf. of the IEEE. IEEE, 2008, pp. 315-318. 